\title{
Free Energies and Fluctuations for the Unitary Brownian Motion
}

\section{Antoine Dahlqvist}

Statistical Laboratory, Centre for Mathematical Sciences, Wilberforce Road, Cambridge, CB3 0WA, UK. E-mail: ad814@maths.cam.ac.uk

Received: 20 October 2014 / Accepted: 6 July 2016

Published online: 29 September 2016 - (C) The Author(s) 2016. This article is published with open access at Springerlink.com

\begin{abstract}
We show that the Laplace transforms of traces of words in independent unitary Brownian motions converge towards an analytic function on a non trivial disc. These results allow one to study the asymptotic behavior of Wilson loops under the unitary Yang-Mills measure on the plane with a potential. The limiting objects obtained are shown to be characterized by equations analogue to Schwinger-Dyson's ones, named here after Makeenko and Migdal.
\end{abstract}

\section{Contents}

1. Introduction . . . . . . . . . . . . . . . . . . 395

2. Unitary Brownian Motion and its Large $N$ Limit . . . . . . . . . . . . . . 398

3. Free Energy, Words in Unitary Brownian Motions . . . . . . . . . . . . . 402

4. Two Estimates on the Cumulants . . . . . . . . . . . . . . . . . . . 410

5. Applications ....................... . 418

6. Planar Yang-Mills Measure . . . . . . . . . . . . . . . . . . . . . 421

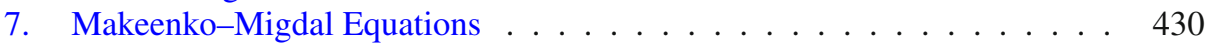

References ....................... . . . 443

\section{Introduction}

The following paper aims at studying traces of non-commutative polynomials in independent Brownian motions on the group of unitary matrices $\mathrm{U}(N)$, as the size $N$ goes to infinity. In $[5,34,35,45]$, it has been shown that for Brownian motions invariant by conjugation, with a proper time-scale, these traces, properly normalized, converge towards a deterministic limit given by the evaluation of the free Brownian motion. We want here to study the Laplace transform of these random variables with normalization analogue to the one of the mod- $\phi$ convergence [22]; it can be viewed as an analogue of the 
well known Harisch-Chandra-Itzykson-Zuber integrals (often abbreviated as HCIZ, $[28,46])$. As a corollary, we obtain the fluctuations of the latter traces around their limit. In [36], the fluctuation of traces in polynomials of one marginal were given, this second point of the present work gives an extension of their result. In [9], Cébron and Kemp have obtained the existence of Gaussian fluctuations of analogue random variables for diffusions on $\mathrm{GL}_{N}(\mathbb{C})$. Therein, the main result is obtained by an exact computation of the moments. In our situation, compactness allows us to go beyond the characterization of fluctuations and to answer analytical questions that are not answered for the HCIZ integrals. A second motivation of our paper is to study the planar Yang-Mills measure for large unitary groups, as well as planar Yang-Mills measure with a potential. We are able here to show the convergence to all orders of the Wilson loops and prove that the limiting objects are characterized by analogues of Schwinger-Dyson equations, named after Makeenko and Migdal. In particular, we prove the existence of a Gaussian field indexed by rectifiable loops describing the fluctuations of the convergence towards the master field proved in [35]. In a subsequent joint work [12], we have obtained bounds on the speed of convergence of moments of a unitary Brownian motion, in order to show the strong convergence of the latter. We believe that an extension of the proof therein leads to larger lower bounds for the radiuses of convergence obtained here and a result of strong convergence for holonomies of rectifiable loops. Though, in order to simplify the presentation, we shall not discuss it further here. Let us also highlight three new proofs of the Makeenko-Migdal equations in [20], discovered later on, during the publication process of the present article. In contrast with the previous ones of [35] and of the current paper, the arguments are local and some of these proofs apply to any compact surface [21].

Free energies of matrix models In many random matrix models, the asymptotic behavior of $\mathbb{E}\left[e^{N \operatorname{Tr}(V)}\right]$, where $V$ is a fixed non-commutative polynomial in a sequence of random matrices of size $N$, have been extensively studied and have several applications ranging from theoretical physics, through enumerative combinatorics, free probability and representation theory. A case of study is the HCIZ integral [13,25, 28,46]

$$
H(A, B)=\mathbb{E}\left[e^{N \operatorname{Tr}\left(A U B U^{*}\right)}\right],
$$

where $A$ and $B$ are two deterministic Hermitian matrices and $U$ is a random unitary matrix, distributed according to the Haar measure. When the non-commutative polynomial plays the role of the potential of a Gibbs measure, the normalized logarithm of Laplace transforms is called the free energy and has been studied in several places, for example in $[7,13,27]$. In the pioneering work [8], formal expansions have been proposed for several physical models. In $[11,14]$, technics have been developed to study formal expansions for model of random matrices with properties of invariance by conjugation. Though, there are yet few results about the radius of convergence of these power series in the complex plane. See [11,13] as well as [24], for a conjecture addressing this question for the Harisch-Chandra-Itzykson-Zuber integrals. We have managed here to give a converging expansion for the following model.

Let $\left(U_{1, t_{1}}, \ldots, U_{q, t_{q}}\right)_{t \in \mathbb{R}_{+}^{q}}$ be $q$ independent Brownian motions invariant by adjunction in $\mathrm{U}(N)$ (see section 2 for a definition) and denote by $\operatorname{Tr}$ the usual non-normalized trace of matrices.

Theorem 1. For $t \in \mathbb{R}_{+}^{q}$ and any non-commutative polynomial $V$ in $2 q$ variables, there exists $r_{V}>0$ and analytic functions $\varphi_{t, V},\left(\psi_{t, V, N}\right)_{N \geq 1}$ and $\psi_{t, V}$ on $D_{r_{V}}=\{z \in \mathbb{C}$ : $\left.|z|<r_{V}\right\}$, such that 


$$
e^{\psi_{t, V, N}(z)}=\mathbb{E}\left[e^{z N \operatorname{Tr}\left(V\left(U_{i, t_{i}}, U_{i, t_{i}}^{*}, i=1 . . q\right)\right)-N^{2} \varphi_{t, V}(z)}\right] \longrightarrow e^{\psi_{t, V}(z)},
$$

as $N \rightarrow \infty$, where the convergence is uniform on compact subset of $D_{r_{V}}$.

For any non-commutative polynomial $V$ in $2 q$ variables, whose restriction to unitary matrices is Hermitian-valued, we shall define for any integer $N \geq 1$, a probability measure $\mu_{N, V}$ on $\mathrm{U}(N)^{q}$ that is absolutely continuous with respect to the law of $\left(U_{i, t_{i}}\right)_{1 \leq i \leq q}$, with density proportional to $e^{z N \operatorname{Tr}\left(V\left(U_{i, t_{i}}, U_{i, t_{i}}^{*}, i=1 . . q\right)\right)}$. Then, for any $N \geq 1$, $\left(U_{N, 1}^{V}, \ldots, U_{N, q}^{V}\right)$ denotes a random variable with law $\mu_{N, V}$.

Theorem 2. If $V, W \in \mathbb{C}\left\langle X_{i}, Y_{i}\right\rangle_{i=1 . . q}$ are non-commutative polynomials with small enough coefficients and $V^{*}=V$, then, under the probability measure $\mu_{N, V}$, the random variable $\frac{1}{N} \operatorname{Tr}\left(W\left(U_{i}^{V}, U_{i}^{V^{*}}, i=1 . . q\right)\right)$ converges in probability towards a constant $\Phi_{t, V}(W)$.

Yang-Mills measure on the plane We shall see that this result can be partly extended to the framework of Yang-Mills measure that has been developed in [1,19,35,37,42]. Therein, we give a recursive way to compute coefficients of $\varphi_{t, V}(z)$, proving analogues of Schwinger-Dyson equations, called here Makeenko-Migdal equations. The latter equations for the first coefficient in $z$ appeared in [38] and were first proved rigorously in [35]. The Yang-Mills measure encompasses the different models for all $q \in \mathbb{N}^{*}$ and $t \in \mathbb{R}_{+}^{q}$, into one random object, for which the recursive equations have simple interpretation. We shall use the approach of [35,37] by considering for any $N \geq 1$, a process $\left(H_{l}\right)_{l}$ indexed by the set $\mathrm{L}\left(\mathbb{R}^{2}\right)$ of rectifiable loops in the plane, valued in $\mathrm{U}(N)$, whose law will be denoted by $\mathrm{YM}_{N}$.

Planar master field The works $[1,35]$ proved that under $\mathrm{YM}_{N}$, the random field $\left(\frac{1}{N} \operatorname{Tr}\left(H_{l}\right)\right)_{l \in \mathrm{L}\left(\mathbb{R}^{2}\right)}$ converges in probability towards a deterministic field $(\Phi(l))_{l \in \mathrm{L}\left(\mathbb{R}^{2}\right)}$. The statement of this result first appeared in the physics literature, in the study of QCD, with the works [31,32,38], and in the mathematical paper [43], as a conjecture. The limiting field was named therein master field, following the terminology of [29]. This object is the first coefficient of an analytic function, limit of Laplace transforms appearing in a generalization of 1 . The asymptotic of $2 D$-Yang-Mills measure on other compact surfaces has also been investigated in the physics literature [26]. It won't be discussed in this text but could lead to future works.

Fluctuations The study of fluctuations of traces of random elements of a compact group of large dimension started with [18], where it was investigated, thanks to representation theory tools, for the Haar measure on the classical compact Lie groups. Theorem 1 allows us in particular to characterize the fluctuations in the convergence of the non-commutative distribution of a $\mathrm{U}(N)$-Brownian motion towards the free unitary Brownian motion distribution. We further prove that under $\mathrm{YM}_{N}$ the random field $\left(\operatorname{Tr}\left(H_{l}\right)-\mathbb{E}\left[\operatorname{Tr}\left(H_{l}\right)\right]\right)_{l \in \mathrm{L}\left(\mathbb{R}^{2}\right)}$ converges in law towards a Gaussian field $\left(\phi_{l}\right)_{l \in \mathrm{L}\left(\mathbb{R}^{2}\right)}$, characterized by the Makeenko-Migdal equations. Besides, we observe that when the loops are dilated by a factor $\lambda$, the above fields have the same Gaussian behavior as $\lambda \rightarrow 0$. Our result extends the work of [36], which studies the Gaussian fluctuations in the convergence of the empirical measure of a $\mathrm{U}(N)$-Brownian motion marginal. The Gaussian field obtained therein can be shown to be a deformation of the one obtained in [18]. The fluctuation results presented in this text are extracted from the $\mathrm{PhD}$ thesis of the author, where the case of the orthogonal and symplectic groups have also been addressed. Note also that in $[3,17]$, fluctuations with another scaling are considered to 
study finite blocks of a random matrix. For the sake of simplicity, we shall restrict here to the study of traces of words in the unitary case.

Organisation of the paper The next section is devoted to the description of the convention we use for the standard Brownian motion on $\mathrm{U}(N)$ and the choice of scaling we made. In Sects. 3 and 4, we obtain the main expressions and estimates needed to get our result. In Sects. 5 and 6, we give their applications to study respectively the unitary Brownian motion and the Yang-Mills measure. In the last section, we show that the limited object obtained in the paper can be characterized by the recursive equations of Makeenko and Migdal.

\section{Unitary Brownian Motion and its Large $N$ Limit}

2.1. Definition and time scale of unitary Brownian motion. For any integer $N$, we shall write $\mathrm{U}(N)$ for the group of unitary matrices of $M_{N}(\mathbb{C})$ and $\mathfrak{u}(N)$ for its Lie algebra, that is, the set of skew-Hermitian matrices. We define a scalar product $\langle\cdot, \cdot\rangle$ on $\mathfrak{u}(N)$ by setting for any $X, Y \in \mathfrak{u}(N)$,

$$
\langle X, Y\rangle=-N \operatorname{Tr}(X Y)
$$

Let us write $\left(K_{t}\right)_{t \geq 0}$ the Brownian motion on the Euclidean space $(\mathfrak{u}(N),\langle\cdot, \cdot\rangle)$ and recall that it is a Gaussian process such that for any $X, Y \in \mathfrak{u}(N), t, s \geq 0$,

$$
\mathbb{E}\left[\left\langle X, K_{t}\right\rangle\left\langle Y, K_{s}\right\rangle\right]=\langle X, Y\rangle \min (t, s) .
$$

Let us define $\left(U_{t}\right)_{t \geq 0}$ as the $M_{N}(\mathbb{C})$-valued solution of the following stochastic differential equation:

$$
\begin{aligned}
d U_{t} & =U_{t} d K_{t}-\frac{1}{2} U_{t} d t \\
U_{0} & =\mathrm{Id}
\end{aligned}
$$

Lemma 1. (i) Almost surely, for all $t \geq 0, U_{t} \in \mathrm{U}(N)$.

(ii) For all $T \geq 0,\left(U_{T}^{*} U_{T+t}\right)_{t \geq 0}$ is independent of the sigma field $\sigma\left(U_{s}, s \leq T\right)$ and has the same law as $\left(U_{t}\right)_{t \geq 0}$.

(iii) For any $t \geq 0$ and every fixed $U \in \mathrm{U}(N), U U_{t} U^{-1}$ has the same law as $U_{t}$.

Proof. Let us prove the first point, the two others are left to the Reader. The processes $\left(i \sqrt{N}\left(K_{t}\right)_{p, p}\right)_{t \geq 0}$ for $1 \leq p \leq N$ and $\left(\sqrt{N}\left(K_{t}\right)_{i, j}\right)_{t \geq 0}$ for $1 \leq i<j \leq N$ are $N^{2}$ independent processes, the $N$ first have the same law as standard real Brownian motions, whereas the others are distributed as standard complex Brownian motions, so that $\mathbb{E}\left[\left|\left(K_{1}\right)_{1,2}\right|^{2}\right]=1$. Let us denote by $\langle\langle\cdot\rangle\rangle$ the symbol of quadratic variations, so that

$$
\left\langle\left\langle d K_{t} . d K_{t}\right\rangle\right\rangle=\sum_{1 \leq i, p, j \leq N}\left\langle\left\langle d\left(K_{t}\right)_{i, p} d\left(K_{t}\right)_{p, j}\right\rangle\right\rangle E_{i, j}=-d t \mathrm{Id} .
$$

Itô's formula then yields

$$
\left.d\left(U_{t} U_{t}^{*}\right)=U_{t}\left(d K_{t}+d K_{t}^{*}\right) U_{t}^{*}+U_{t}\left(\left\langle d K_{t} \cdot d K_{t}^{*}\right\rangle\right\rangle-d t \mathrm{Id}\right) U_{t}^{*}=0 .
$$


We call this process the $\mathrm{U}(N)$-Brownian motion $^{1}$ (see $[16,35]$ for a similar definition on other classical compact groups). For $N=1$, it has the same law as $\left(e^{i B_{t}}\right)_{t \geq 0}$, where $\left(B_{t}\right)_{t \geq 0}$ is the standard real Brownian motion. Let us make remarks on the scaling. Recall that the scalar product $\langle\cdot, \cdot\rangle$ on $\mathfrak{u}(N)$ induces a Riemannian metric $d$ on $\mathrm{U}(N)$. On the one hand, this choice of metric yields that the diameter of $\mathrm{U}(N)$ is $d(\mathrm{Id},-\mathrm{Id})=\int_{0}^{1}\left\|\dot{\gamma}_{t}\right\| d t$, where $\gamma: t \in[0,1] \mapsto \exp \left(t i \pi \mathrm{Id}_{N}\right)$, that is, $\|i \pi \mathrm{Id}\|=N \pi$. On the other hand, the law of large numbers implies that $\operatorname{dim}(\mathfrak{u}(N))^{-1}\left\|K_{t}\right\|^{2}=N^{-2}\left\|K_{t}\right\|^{2}$ converges, as $N \rightarrow \infty$, towards $t$. Heuristically, we may infer that, as $N \rightarrow \infty$, for any $t>0, d\left(\mathrm{Id}, U_{t}\right)$ behaves like $\left\|K_{t}\right\|$ and $\frac{d\left(U_{t}, \text { Id }\right)}{d(\mathrm{Id},-\mathrm{Id})} \rightarrow C_{t} \in(0, \infty)$. With this scaling, the Brownian motion "has the time to visit" $2 \mathrm{U}(N)$. Besides, the stochastic differential equation (*) does not depend on $N$ and such an equation makes sense in the context of free stochastic differential equations (see [5]). Let us add a last comment on the time-scale. With the above choice, the $\mathrm{U}(1)$-Brownian motion appears with the same scaling in all $\mathrm{U}(N)$-Brownian motions.

Lemma 2. For any $N \in \mathbb{N}^{*}$, let $\left(U_{t, N}\right)_{t \geq 0}$ be a $\mathrm{U}(N)$-Brownian motion. Then, the process $\left(\operatorname{det}\left(U_{t, N}\right)\right)_{t \geq 0}$ has the same distribution as $\left(\mathrm{U}_{t, 1}\right)_{t \geq 0}$.

Proof. Observe that for any $N \in \mathbb{N}^{*},\left(i \operatorname{Tr}\left(K_{t}\right)\right)_{t \geq 0}$ has the same law as a standard Brownian motion. If $D_{2}(\mathrm{det})_{M}: M_{N}(\mathbb{C})^{2} \rightarrow \mathbb{C}$ denotes the second derivative of the determinant at a point $M \in M_{N}(\mathbb{C})$, Itô's formula yields that

$$
d\left(\operatorname{det}\left(U_{t}\right)\right)=\operatorname{det}\left(U_{t}\right) d \operatorname{Tr}\left(K_{t}\right)-\frac{N}{2} \operatorname{det}\left(\mathrm{U}_{t}\right) d t+\operatorname{det}\left(U_{t}\right)\left\langle\left\langle D_{2}(\operatorname{det})_{\operatorname{Id}}\left(d K_{t}, d K_{t}\right)\right\rangle\right\rangle .
$$

What is more,

$$
\begin{aligned}
\left\langle\left\langle D_{2}(\mathrm{det})_{\operatorname{Id}}\left(d K_{t}, d K_{t}\right)\right\rangle\right\rangle & =\sum_{1 \leq i<j \leq N}\left(\left\langle\left\langle\left(d K_{t}\right)_{i, i},\left(d K_{t}\right)_{j, j}\right\rangle\right\rangle-\left\langle\left\langle\left(d K_{t}\right)_{j, i},\left(d K_{t}\right)_{i, j}\right\rangle\right\rangle\right) \\
& =\frac{N(N-1)}{2 N} d t .
\end{aligned}
$$

Hence, $\left(\operatorname{det}\left(U_{t}\right)\right)_{t \geq 0}$ is the unique strong solution to

$$
d\left(\operatorname{det}\left(U_{t}\right)\right)=\operatorname{det}\left(U_{t}\right) d\left(\operatorname{Tr}\left(K_{t}\right)\right)-\frac{1}{2} \operatorname{det}\left(U_{t}\right) d t
$$

and $\operatorname{det}\left(U_{0}\right)=1$, that is $\left(\exp \left(\operatorname{Tr}\left(K_{t}\right)\right)\right)_{t \geq 0}$.

2.2. Free unitary Brownian motion. Let us recall the first result obtained about the behavior of unitary Brownian motion in large dimension. We shall denote by $\left(\mu_{t}^{N}\right)_{t \geq 0}$ the family of random measures given by the empirical measure of eigenvalues of $\mathrm{U}_{t}$ : if $\lambda_{1}, \ldots, \lambda_{N} \in \mathbb{U}$ are the eigenvalues of $\mathrm{U}_{t}, \mu_{t}^{N}=\frac{1}{N}\left(\delta_{\lambda_{1}}+\cdots+\delta_{\lambda_{N}}\right)$. Note that for any $P \in \mathbb{C}[X], \frac{1}{N} \operatorname{Tr}\left(P\left(\mathrm{U}_{t}\right)\right)=\int_{\mathbb{U}} P(z) \mu_{t}(d z)$. The following theorem has first been proved in [5] using harmonic analysis on the unitary group and by [40] using stochastic calculus.

\footnotetext{
1 It can be shown that it is a diffusion on the Riemannian manifold $\mathrm{U}(N)$ endowed by the left-invariant metric associated to $\langle\cdot, \cdot\rangle$ and that its generator is the Laplace-Beltrami operator (see [36,44], Proposition 2.1., for an elementary proof).

2 Note that a good scaling to study the convergence of the distance in total variation $d_{T V}$ between the law of Brownian motion and the Haar measure, is faster than ours. Let $U$ be a Haar distributed random variable on $\mathrm{U}(N)$. It has been shown in [39] that the function $t \mapsto d_{T V}\left(U_{t} \log (N), U\right)$ admits a cut-off around the value $t=2$.
} 
Theorem $3[5,34,40,45]$. The sequence or random measures $\left(\mu_{t}^{N}\right)_{N \geq 0}$ converges weakly in probability, ${ }^{3}$ towards a deterministic measure $\mu_{t}$ on $\mathbb{U}$, whose moments are given as follows:

$$
\mu_{t, n}=\int_{\mathbb{U}} z^{n} \mu_{t}(d z)=e^{-\frac{n t}{2}} \sum_{k=0}^{n-1} \frac{(-t)^{k}}{k !} n^{k-1}\left(\begin{array}{c}
n \\
k+1
\end{array}\right) .
$$

Using the property of independence and stationarity satisfied by multiplicative increments of a $\mathrm{U}(N)$-Brownian motion, together with the invariance of their law by adjunction, free probability arguments lead to the following Theorem.

Theorem 4 [5]. For any $t_{1}, t_{2}, \ldots, t_{q} \geq 0$ and $V$ any non-commutative polynomial in $2 q$-variables, the random variables $\frac{1}{N} \operatorname{Tr}\left(V\left(U_{1}, U_{1}^{*}, \ldots, U_{q}, U_{q}^{*}\right)\right)$ converge in probability towards a constant.

The limiting object is called the non-commutative distribution of the free unitary Brownian motion and can be characterized by the family of measures $\left(\mu_{t}\right)_{t \geq 0}$ together with the asymptotic freeness of the increments. This last theorem was proved in another way in $[34,35,45]$ showing directly the convergence for any non-commutative polynomial. Let us recall how the argument of $[34,35]$ goes to show Theorem 3 . Let us denote by $\mathfrak{S}_{n}$ the group of permutations of

$$
[n]:=\{1, \ldots, n\} .
$$

For any permutation $\sigma \in \mathfrak{S}_{n}$ composed of \# $\sigma$ cycles, we define a function $f_{\sigma}$ on $\mathrm{U}(N)$ by setting for any $U \in \mathrm{U}(N)$,

$$
f_{\sigma}(U)=N^{-\# \sigma} \operatorname{Tr}\left(\sigma U^{\otimes n}\right)
$$

and a function on $\mathfrak{S}_{n}$, by setting for any $t>0$,

$$
\varphi_{t}^{N}(\sigma)=\mathbb{E}\left[f_{\sigma}\left(U_{t}\right)\right],
$$

where $U_{t}$ is the marginal of a $\mathrm{U}(N)$-Brownian motion. Then, the latter family of functions on the symmetric group is shown to satisfy the following differential system (see $[34,40]$ or Lemma 4).

Lemma 3 [34,40]. For any permutation $\sigma \in \mathfrak{S}_{n}$,

$$
\begin{aligned}
\frac{d}{d t} \varphi_{t}^{N}(\sigma) & =-\frac{n}{2} \varphi_{t}^{N}(\sigma)-\sum_{1 \leq i<j \leq n} N^{\# \sigma(i j)-\# \sigma-1} \varphi_{t}^{N}(\sigma(i j)), \\
\varphi_{0}^{N}(\sigma) & =1 .
\end{aligned}
$$

The unique solution of this system of ordinary differential equations is a power series in $\frac{1}{N}$ that converges, as $N \rightarrow \infty$, to a function $\varphi_{t}$. It can further be shown to satisfy for any $\sigma \in \mathfrak{S}_{n}$ with $a_{k}$ cycles of length $k$,

$$
\varphi_{t}(\sigma)=\prod_{k=1}^{n} \varphi_{t}((1 \ldots k))^{a_{k}}
$$

\footnotetext{
3 We mean here that for any continuous function $f$, the sequence of random variables $\left(\int f d \mu_{t}^{N}\right)_{N \geq 1}$ converges in probability to the constant $\int f d \mu_{t}$.
} 
Setting for all $t \geq 0, n \geq 1, \mu_{t, n}=\varphi_{t}((1 \ldots n))$, the limit in $N$ of the former equations takes the following form:

$$
\frac{d}{d t} \mu_{t, n}=-\frac{n}{2} \mu_{t, n}-\frac{n}{2} \sum_{k=1}^{n-1} \mu_{t, k} \mu_{t, n-k}
$$

with initial condition $\mu_{0, n}=1$. This system of equations is then shown to have a unique solution given by the expression of Theorem [5]. It follows that for $n \in \mathbb{N}, t \geq 0$,

$$
\mathbb{E}\left[\int_{\mathbb{U}} \omega^{n} \mu_{t}^{N}(d \omega)\right] \rightarrow \mu_{t, n}
$$

To conclude and obtain a convergence in probability, one ultimately needs to estimate the covariances of the complex variables $\left(\frac{1}{N} \operatorname{Tr}\left(U_{t}^{n}\right)\right)_{n \in \mathbb{N}, t>0}$ with their complex conjugate. This latter point together with the Lemma 3 can be proved using the following lemma, that allows to study any polynomial in the entries and their conjugate of a unitary Brownian motion.

For any integer $n \in \mathbb{N}^{*}$, let us recall the left action of $\mathfrak{S}_{n}$ on $\mathbb{C}^{\otimes n}$, such that for any permutation $\sigma \in \mathfrak{S}_{n}$ and any elementary tensor $v_{1} \otimes v_{2} \otimes \ldots \otimes v_{n} \in\left(\mathbb{C}^{N}\right)^{\otimes n}$,

$$
\sigma . v_{1} \otimes v_{2} \otimes \cdots \otimes v_{n}=v_{\sigma^{-1}(1)} \otimes \cdots v_{\sigma^{-1}(n)} .
$$

The endomorphism of $\left(\mathbb{C}^{N}\right)^{\otimes n}$ associated to a permutation $\sigma$ will be abusively denoted below by the same symbol. For any pair of distinct integers $i, j \in[n]$, we denote by $\langle i j\rangle$ the endomorphism of $\left(\mathbb{C}^{N}\right)^{\otimes n}$ which acts like the endomorphism $\sum_{1 \leq r, s \leq N} E_{r, s} \otimes E_{r, s}$ on the $i$ th and $j$ th tensors and trivially on the others.

Lemma $4[16,35]$. Let $U_{t}$ be a Brownian motion on $\mathrm{U}(N)$. For any positive integers $a, b$, which add up to $n$, the following differential equation holds:

$$
\frac{d}{d t} \mathbb{E}\left[U_{t}^{\otimes a} \otimes \bar{U}_{t}^{\otimes b}\right]=-\mathbb{E}\left[U_{t}^{\otimes a} \otimes \bar{U}_{t}^{\otimes b}\right]\left(\frac{n}{2}+\frac{1}{N} \sum_{\substack{i<j \leq a \\ \text { or } a<i<j}}(i j)-\frac{1}{N} \sum_{i \leq a<j}\langle i j\rangle\right) .
$$

For any permutation $\sigma \in \mathfrak{S}_{n}, \varphi_{t}^{N}(\sigma)=N^{-\# \sigma} \operatorname{Tr}\left(\sigma \mathbb{E}\left[U_{t}^{\otimes n}\right]\right)$ and the Lemma 3 reduces to this more general one.

Proof. We shall use the stochastic differential equation $\left(^{*}\right)$ and apply Itô formula. First, writing the $\mathfrak{u}(N)$-valued Brownian motion $\left(K_{t}\right)_{t \geq 0}$ as a sum of independent real standard Brownian motions yields that

$$
\left\langle\left\langle d K_{t} \otimes d K_{t}\right\rangle\right\rangle=-\frac{1}{N} \sum_{1 \leq r, s \leq N} E_{r, s} \otimes E_{s, r} d t=-\frac{1}{N}(12) d t \in \operatorname{End}\left(\left(\mathbb{C}^{N}\right)^{\otimes 2}\right)
$$

and

$$
\left\langle\left\langle d K_{t} \otimes d \bar{K}_{t}\right\rangle\right\rangle=\frac{1}{N} \sum_{1 \leq r, s \leq N} E_{r, s} \otimes E_{r, s}=\frac{1}{N}\langle 12\rangle d t .
$$


We can now use the Ito formula to get that the variational-bounded part of the variation of the semi-martingales $U_{t}^{\otimes 2}$ and $U_{t} \otimes \overline{U_{t}}$ are respectively $U_{t} \otimes U_{t} \cdot\left(-d t+\left\langle\left\langle d K_{t} \otimes d K_{t}\right\rangle\right\rangle\right)=$ $-U_{t}^{\otimes 2}\left(1+\frac{1}{N}\left(\begin{array}{ll}1 & 2))\end{array}\right) d t\right.$ and $U_{t} \otimes \overline{U_{t}}\left(-d t+\left\langle\left\langle d K_{t} \otimes d \bar{K}_{t}\right\rangle\right\rangle\right)=-U_{t} \otimes \overline{U_{t}}\left(1-\frac{1}{N}\langle 12\rangle\right) d t$. The same analysis yields that the variational-bounded part of the variation of the semimartingale $U_{t}^{\otimes a} \otimes \bar{U}_{t}^{\otimes b}$ is

$$
U_{t}^{\otimes a} \otimes \bar{U}_{t}^{\otimes b}\left(-\frac{n}{2}-\frac{1}{N} \sum_{i<j \leq a, \text { or } a<i<j}(i j)+\frac{1}{N} \sum_{i \leq a<j}\langle i j\rangle\right) d t .
$$

\section{Free Energy, Words in Unitary Brownian Motions}

The convergence of the above paragraph can be considered as a law of large numbers for the traces of words in unitary Brownian motion. We aim at studying their Laplace transform and at deriving from this study a central limit theorem. Note that if $\left(K_{t}\right)_{t \geq 0}$ is a $\mathfrak{u}(N)$ Brownian motion as defined above, for any $t \geq 0, N^{-2} \log \mathbb{E}\left[e^{N \operatorname{Tr}\left(K_{t}\right)}\right]=$ $N^{-2} \log \mathbb{E}\left[e^{i\left\langle K_{t}, i \mathrm{Id}_{N}\right\rangle}\right]=-t$ and $N^{-2} \log \mathbb{E}\left[e^{N \operatorname{Tr}\left(K_{t} K_{t}^{*}\right)}\right]=N^{-2} \log \mathbb{E}\left[e^{-\left\|K_{t}\right\|^{2}}\right]=$ $\log \mathbb{E}\left[e^{-B_{t}^{2}}\right]$, where $B_{t}$ is the marginal of a standard real Brownian motion. These two naive examples suggest that the scaling chosen in Theorem 1 is the good one (see [47] for examples of Hermitian matrix models, where this scaling is used). We shall prove it in the following by estimating cumulants.

\subsection{Laplace transforms and cumulants of traces.}

3.1.1. Scaling of cumulants. For any bounded random variable $X$, the function $\log \mathbb{E}\left[e^{z X}\right]$ is analytic on a neighborhood of 0 . We denote its analytic expansion

$$
\log \mathbb{E}\left[e^{z X}\right]=\sum_{n \geq 1} \frac{C_{n}(X)}{n !} z^{n}
$$

the coefficients $\left(C_{n}(X)\right)_{n \geq 1}$ are called the classical cumulants of the random variable $X$. We are interested here in the behavior in $N$ of $N^{-2} \log \mathbb{E}\left[e^{N \operatorname{Tr}\left(A_{N}\right)}\right]$, hence of the rescaled cumulant

$$
N^{n-2} C_{n}\left(\operatorname{Tr}\left(A_{N}\right)\right),
$$

where the $A_{N}$ are random matrices of $M_{N}(\mathbb{C})$, uniformly bounded in norm.

3.1.2. Cumulants of several random variables. These coefficients are related to the moments of $X$ via a Möbius inversion formula ([41], [11,2.3]) in a lattice of partitions. For any $n \in \mathbb{N}^{*}$, the set $\mathcal{P}_{n}$ of partitions of $[n]$ is endowed with a partial order $\preccurlyeq$, such that for $\pi, \nu \in \mathcal{P}_{n}, \pi \preccurlyeq v$ if the blocks of $\pi$ are included in the ones of $\nu$. It has a maximum and a minimum that we denote respectively by $1_{n}$ and $0_{n}$. For any pair $\pi, \nu \in \mathcal{P}_{n}, \pi \wedge \nu$ (respectively $\pi \vee v$ ) denotes the biggest (smallest) partition smaller (bigger) than $\pi$ and $\nu$. Each partition $\pi$ has $\# \pi$ blocks. For any sequence of complex numbers $\left(\alpha_{A}\right)_{A \subset[n]}$, let us set for any partition $\pi \in \mathcal{P}_{n}$, 


$$
\alpha_{\pi}=\prod_{A \in \pi} \alpha_{A} .
$$

Then, for any $\pi \in \mathcal{P}_{n}$, there exists a unique sequence $\left(\beta_{\pi, v}(\alpha)\right)_{\pi \preccurlyeq v}$ such that for any $v \succcurlyeq \pi$

$$
\alpha_{\nu}=\sum_{\pi \preccurlyeq \pi^{\prime} \preccurlyeq v} \beta_{\pi, \pi^{\prime}}(\alpha) .
$$

For any $\pi, v \in \mathcal{P}_{n}$ with $v \preccurlyeq \pi$ and $\pi \neq v$, we set $\beta_{\pi, v}(\alpha)=0$. If $X_{1} \ldots, X_{n}$ are bounded complex random variables and for any $A \subset[n], \alpha_{A}=\mathbb{E}\left[\prod_{i \in A} X_{i}\right]$, let us set $C_{n}\left(X_{1}, \ldots, X_{n}\right)=\beta_{0_{n}, 1_{n}}(\alpha)$ and similarly, for any pair $(\pi, \nu)$ of partitions, $C_{\pi, v}\left(X_{1}, \ldots, X_{n}\right)=\beta_{\pi, v}(\alpha)$. Then, the following expansion holds for any $z \in \mathbb{C}^{n}$ in a neighborhood of 0 ,

$$
\log \mathbb{E}\left[e^{z_{1} X_{1}+\cdots+z_{n} X_{n}}\right]=\sum_{\substack{k \geq 1 \\ 1 \leq i_{1}, \ldots, i_{k} \leq n}} C_{k}\left(X_{i_{1}}, \ldots, X_{i_{k}}\right) \frac{z_{i_{1}} \ldots z_{i_{k}}}{k !}
$$

Differentiating the previous expression leads to two useful formulas: if $Y$ and $Z$ are bounded random variables coupled with $X$ and if $z \in \mathbb{C}$ is in a neighborhood of 0 , then

$$
\frac{\mathbb{E}\left[Y e^{z X}\right]}{\mathbb{E}\left[e^{z X}\right]}=\sum_{k \geq 0} \frac{C_{k+1}(Y, X, \ldots, X)}{k !} z^{k}
$$

and

$$
\frac{\mathbb{E}\left[Y Z e^{z X}\right]}{\mathbb{E}\left[e^{z X}\right]}-\frac{\mathbb{E}\left[Y e^{z X}\right] \mathbb{E}\left[Z e^{z X}\right]}{\mathbb{E}\left[e^{z X}\right]^{2}}=\sum_{k \geq 0} \frac{C_{k+2}(Y, Z, X, \ldots, X)}{k !} z^{k} .
$$

The coefficient $C_{n}\left(X_{1}, \ldots, X_{n}\right)$ is called a classical cumulant and is symmetric in the variables $X_{1}, \ldots, X_{n}$. The coefficients $\left(C_{\pi, v}\left(X_{1}, \ldots, X_{n}\right)\right)_{\pi \preccurlyeq v}$ are called relative cumulants. Using the characterizing formula (2), one can express relative cumulants in terms of classical cumulants. For any pair $\pi \preccurlyeq v$, if for any $A \in v, A_{\pi}$ denotes the set of blocks of $\pi$ included in $A$, then

$$
\begin{aligned}
C_{\pi, v}\left(X_{1}, \ldots, X_{n}\right) & =\prod_{A \in v} C_{\# A_{\pi}}\left(\prod_{i \in B} X_{i}, B \in A_{\pi}\right) \\
& =\sum_{\mu \in \mathcal{P}_{n}: \pi \vee \mu=v} C_{\mu}\left(X_{1}, \ldots, X_{n}\right) .
\end{aligned}
$$

The second formula is named after Leonov and Shiryaev [33].

3.1.3. Tensor valued cumulants. We shall here recall the notion of moments and cumulants for vector valued random variables (see [15], where this notion is considered in the broader setting of non-commutative probability spaces). This framework will later on (Sect. 4.2) allow us to obtain representation formulas for classical cumulants in terms of matrices, which are the core of the argument to bound from below the radius of convergence of Laplace transforms.

For any finite dimensional vector space $V$ and any finite set $A$, let us denote by $V^{\otimes A}$ the vector space of multilinear map on $\left(V^{*}\right)^{A}$ and for any $n \in \mathbb{N}^{*}$ identify $V^{\otimes[n]}$ with $V^{\otimes n}$. Any function $X:[n] \rightarrow V$ defines an elementary element of $V^{\otimes A}$ that we 
denote by $\bigotimes_{i \in A} X_{i}$. Any partition $\pi \in \mathcal{P}_{n}$ defines a multilinear map $\prod_{A \in \pi} V^{\otimes A} \rightarrow$ $V^{\otimes n},\left(\alpha_{A}\right)_{A \in \pi} \mapsto \bigotimes_{A \in \pi} \alpha_{A}$, such that for any $X \in V^{n}, \bigotimes_{A \in \pi} \bigotimes_{i \in A} X_{i}=\bigotimes_{i \in[n]} X_{i}$. For instance, for $v_{1}, \ldots, v_{4} \in V$, if $\alpha_{\{1,3\}}=v_{1} \otimes v_{2} \in V^{\otimes 2} \simeq V^{\otimes\{1,3\}}$ and $\alpha_{\{2,3\}}=$ $v_{3} \otimes v_{4} \in V^{\otimes 2} \simeq V^{\otimes\{2,4\}}$,

$$
\bigotimes_{A \in\{\{1,3\},\{2,4\}\}} \alpha_{A}=v_{1} \otimes v_{3} \otimes v_{2} \otimes v_{4}
$$

For any sequence $\left(\alpha_{A}\right)_{A \subset[n]}$ such that for any $A \subset[n], \alpha_{A} \in V^{\otimes A}$, let us set for any partition $\pi \in \mathcal{P}_{n}$,

$$
\alpha_{\pi}=\bigotimes_{A \in \pi} \alpha_{A} \in V^{\otimes n}
$$

Then, for any $\pi \in \mathcal{P}_{n}$, there exists a unique sequence $\left(\beta_{\pi, v}(\alpha)\right)_{\pi \preccurlyeq v}$ such that for any $v \succcurlyeq \pi$,

$$
\alpha_{v}=\sum_{\pi \preccurlyeq \pi^{\prime} \preccurlyeq v} \beta_{\pi, \pi^{\prime}}(\alpha) \in V^{\otimes n} .
$$

When $X_{1} \ldots, X_{n}$ are bounded random variables valued in $V$ on a probability space $(\Omega, \mathcal{B}, \mathbb{P})$ and for any $A \subset[n], \alpha_{A}=\mathbb{E}\left[\bigotimes_{i \in A} X_{i}\right]$, note that for any pair of partitions $\pi, v, C_{\pi, v}\left(X_{1}, \ldots, X_{n}\right)=\beta_{\pi, v}(\alpha)$ is $n$-linear as a function on the space $\mathrm{L}^{\infty}(\Omega, \mathcal{B}, \mathbb{P}) \otimes$ $V$. We then define linear functions on $\mathrm{L}^{\infty}(\Omega, \mathcal{B}) \otimes V^{\otimes n}$ by setting for any random variables $X_{1}, \ldots, X_{n} \in \mathrm{L}^{\infty}(\Omega, \mathcal{B}, \mathbb{P}) \otimes V, C_{\pi, v}\left(X_{1} \otimes \cdots \otimes X_{n}\right)=C_{\pi, v}\left(X_{1}, \ldots, X_{n}\right)$ and $C_{n}\left(X_{1} \otimes \cdots \otimes X_{n}\right)=C_{0_{n}, 1_{n}}\left(X_{1}, \ldots, X_{n}\right)$. For example, if $A$ and $B$ are two bounded random vectors of $V$,

$$
\begin{gathered}
C_{2}(A \otimes B)=\mathbb{E}[A \otimes B]-\mathbb{E}[A] \otimes \mathbb{E}[B] \in V^{\otimes 2}, \\
C_{\{\{1,2\},\{3\}\}, 1_{3}(A \otimes B \otimes C)}=\mathbb{E}[A \otimes B \otimes C]-\mathbb{E}[A \otimes B] \otimes \mathbb{E}[C] \in V^{\otimes 3} .
\end{gathered}
$$

If $A_{1}, \ldots, A_{n}$ are random matrices in $M_{n}\left(\mathbb{C}^{N}\right)$ with bounded operator norms and $\pi, v \in$ $\mathcal{P}_{n}$ is a pair of partitions, then

$$
C_{\pi, v}\left(\operatorname{Tr}\left(A_{1}\right), \operatorname{Tr}\left(A_{2}\right), \ldots, \operatorname{Tr}\left(A_{n}\right)\right)=\operatorname{Tr}_{\left(\mathbb{C}^{N}\right)^{\otimes n}}\left(C_{\pi, v}\left(A_{1} \otimes \cdots \otimes A_{n}\right)\right) .
$$

If $\sigma \in \mathfrak{S}_{n}$ is a permutation whose orbits are included in blocks of the partition $v$, then

$$
C_{\# v}\left(\prod_{\substack{\left(i_{1} \ldots i_{k}\right) \text { cycle of } \sigma \\ \text { included in } B}} \operatorname{Tr}\left(A_{i_{1}} \ldots A_{i_{k}}\right), B \in v\right)=\operatorname{Tr}\left(C_{v, 1_{n}}\left(\sigma A_{1} \otimes A_{2} \otimes \cdots \otimes A_{n}\right)\right) .
$$

3.1.4. Cumulant of exponential tensors. For any $n \in \mathbb{N}^{*}$, let us denote by $\mathfrak{P}_{n}$ the set of subsets of $[n]$. For any $A, B \in \mathfrak{P}_{n}$, with $B \subset A$, let us write $\bar{B}^{A}$ for the smallest partition of $A$ for $\preccurlyeq$, containing $B$ as a block, and set for any endomorphism $T \in \operatorname{End}\left(V^{\otimes B}\right)$, $\bar{T}^{A}=\bigotimes_{S \in\{B, A \backslash B\}} \alpha_{S} \in \operatorname{End}\left(V^{\otimes A}\right)$, where $\alpha_{B}=T$ and $\alpha_{A \backslash B}=\operatorname{Id}_{V \otimes A \backslash B}$. If $A=[n]$, we shall write respectively $\bar{B}$ and $\bar{T}$ for $\bar{B}^{A}$ and $\bar{T}^{A}$. For any $d \geq 1$, let us denote by $\Delta^{d}$ the simplex $\left\{s \in[0,1]^{d+1}: s_{0}+\ldots+s_{d}=1\right\}$ of dimension $d$ and by $d s$ the Lebesgue measure on $\Delta^{d}$. 
Lemma 5. Let $\left(T_{A}\right)_{A \in \mathfrak{P}_{n}, \# A \geq 2}$ be a family of endomorphisms such that for any $A \in \mathfrak{P}_{n}$, $T_{A} \in \operatorname{End}\left(V^{\otimes A}\right)$ and

$$
\alpha_{A}=\exp \left[\sum_{B \subset A, \# B \geq 2}{\overline{T_{B}}}^{A}\right] .
$$

Then, for any pair of partitions $\pi \preccurlyeq v$ in $\mathcal{P}_{n}$,

$$
\beta_{\pi, v}(\alpha)=\sum \int_{\Delta^{d}} \alpha_{\pi}^{s_{0}} \bar{T}_{A_{1}} \alpha_{\pi \vee A_{1}}^{s_{1}} \bar{T}_{A_{2}} \ldots \bar{T}_{A_{d}} \alpha_{v}^{s_{d}} d s
$$

where the sum is over sequences $A_{1}, \ldots, A_{d}$, such that $\left(\pi \vee\left(\vee_{i=1}^{k} A_{i}\right)\right)_{0 \leq k \leq d}$ is strictly increasing, with $\pi \vee A_{1} \vee \ldots \vee A_{d}=v$. Moreover, if $\mu \in \mathcal{P}_{n}$ and for any $t \in \mathbb{R}^{\mu}$,

$$
T_{A}(t)=t_{C} T_{A},
$$

whenever $A \subset C$, with $C \in \mu$, and 0 otherwise, then for any $C \in \mu$,

$$
\frac{d}{d t_{C}} \beta_{\pi, v}(\alpha)=\sum_{A \subset C: \# A \geq 2} \bar{T}_{A} \beta_{\pi \vee A, v}(\alpha(t)) .
$$

Though we shall not use it, it is also enlightening and easy to show that for any partitions $\pi \preccurlyeq v$ of $[n]$,

$$
\beta_{\pi, v}(\alpha)=\sum_{k \geq 0} \frac{1}{k !} \sum \overline{T_{A_{1}}} \ldots \overline{T_{A_{k}}} \in \operatorname{End}\left(V^{\otimes n}\right),
$$

where the second sum is over sequences $A_{1}, \ldots, A_{k} \in \mathfrak{P}_{n}$, with $\# A_{i} \geq 2$ for any $1 \leq i \leq k$ and $\pi \vee A_{1} \vee A_{2} \vee \ldots \vee A_{k}=v$.

Proof. Applying the uniqueness of (7) to the integral of the right-hand-side of (10) implies the latter. Then, (10) for $\mu=1_{n}$, implies that both sides of (9) satisfies the same differential equation. Moreover, both sides have the same initial condition: $\beta_{\pi, v}(\alpha(0))=$ 1 , if $\pi=v$ and 0 otherwise. Hence, by induction on $\# \pi-\# v$, the two terms agree.

3.2. Words in independent unitary Brownian motions and their traces. We obtain here a differential system for the normalized cumulants in traces of words of unitary Brownian motions and show that the latter converge as $N \rightarrow \infty$.

3.2.1. Partitioned words. For each positive integer $q, W_{q}$ denotes the monoid of words in the alphabet made of $2 q$ symbols $x_{1}, \ldots, x_{q}, x_{1}^{-1}, \ldots, x_{q}^{-1}$. An element $w$ of $W_{q}$ writes down uniquely $x_{i_{1}}^{\epsilon_{1}} \ldots x_{i_{n}}^{\epsilon_{n}}$, with $\epsilon_{1}, \ldots, \epsilon_{n} \in\{-1,1\}$. We call $n$ the length of $w$ and denote it by $\ell(w)$. Its $p^{\text {th }}$ letter $x_{i_{p}}^{\epsilon_{p}}$ is denoted by $X_{p}(w)$ and for any $k \in[q]$ and $A \subset[n]$, we set $n_{w, A}^{ \pm}(k)=\#\left\{r \in A: X_{r}(w)=x_{k}^{ \pm}\right\}$,

$$
n_{w, A}(k)=n_{w, A}^{+}(k)-n_{w, A}^{-}(k) \quad \text { and } \quad \bar{n}_{w, A}(k)=n_{w, A}^{+}(k)+n_{w, A}^{-}(k),
$$

where we shall drop the second index when $A=[n]$. We call partitioned word every couple $(S, \pi)$, where $S$ is a tuple $\left(w_{1}, \ldots, w_{m}\right)$ of words in $W_{q}$ and $\pi \in \mathcal{P}_{m}$. Given such a couple, we set $\# S=m, w(S)=w_{1} w_{2} \ldots w_{m}$ and $\ell(S)=\ell(w(S, \pi))$. We denote the set of partitioned words by $\mathcal{P} W_{q}$. For any $m \in \mathbb{N}^{*}$ and $w \in W_{q}, w^{[m]}$ denotes the tuple composed of $m$ copies of $w$. For any function $\varphi \in \mathbb{C}^{\mathcal{P} W_{q}}$, and $S \in W_{q}^{m}$, we shall write $\varphi(S)$ for $\varphi\left(\left(S, 0_{m}\right)\right)$. 
3.2.2. Operations on partitioned words. For any partitioned word $(S, \pi) \in \mathcal{P} W_{q}$ with $S=\left(w_{1}, \ldots, w_{m}\right)$ and $w=w(S, \pi)$, let us introduce two transformations of $(S, \pi)$. For any pair of positive integers $i, j$ such that $1 \leq i<j \leq \ell(S)$ and $X_{i}(w)=X_{j}(w)^{ \pm}=a$, let us define $\mathcal{T}_{i, j}^{ \pm}((S, \pi))=\left(\mathcal{T}_{i, j}^{ \pm}(S), \pi^{\prime}\right) \in \mathcal{P} W_{q}$ according to two cases, as illustrated in Figs. 1, 2.

1. If the $i$ th and $j$ th letters of $w$ belong to the same word $w_{k}=\lambda X_{i}(w) \mu X_{j}(w) v$, then let us set

$$
\mathcal{T}_{i, j}^{+}(S)=\left(w_{1}, \ldots, w_{k-1}, \lambda a v, a \mu, w_{k+1}, \ldots, w_{m}\right),
$$

if $X_{i}(w)=X_{j}(w)$

$$
\mathcal{T}_{i, j}^{-}(S)=\left(w_{1}, \ldots, w_{k-1}, \lambda v, a \mu a^{-1}, w_{k+1}, \ldots, w_{m}\right),
$$

if $X_{i}(w)=X_{j}(w)^{-1}$ and in both cases $\pi^{\prime} \in \mathcal{P}_{m+1}$ the partition obtained from $\pi$ by substituting $l$ with $l+1$ for $l>k$ and adding $k+1$ to any block of $\pi$ including $k$.

2. If the $i$ th and $j$ th letters of $w$ belong to two words $w_{p}=\lambda X_{i}(w) \mu$ and $w_{q}=$ $v X_{j}(w) \chi$, then let us set

$$
\mathcal{T}_{i, j}^{+}(S)=\left(w_{1}, \ldots, \hat{w}_{p}, \lambda a \chi v a \mu, \ldots, \hat{w}_{q}, \ldots, w_{m}\right),
$$

if $X_{i}(w)=X_{j}(w)$

$$
\mathcal{T}_{i, j}^{-}(S)=\left(w_{1}, \ldots, \hat{w}_{p}, \lambda \chi v a^{-1} a \mu, \ldots, \hat{w}_{q}, \ldots, w_{m}\right),
$$

if $X_{i}(w)=X_{j}(w)^{-1}$ and in both cases we let $\pi^{\prime} \in \mathcal{P}_{m-1}$ be the image of the restriction of $\pi$ to $[m] \backslash\{q\}$ by the increasing bijection $[m] \backslash\{q\} \rightarrow[m-1]$.

Let us make two remarks. In the first case the number of blocks of the partitioned words are constant and the number of words is increased by 1 . In the second one, the number of words is decreased by 1 , whereas the number of blocks of $\# \pi^{\prime}$ is equal to $\# \pi$, if $p$ and $q$ belong to the same block of $\pi$ and $\# \pi-1$ otherwise. For any $f \in[q]$, we define the sets

$$
\begin{aligned}
\mathcal{N}_{w}^{ \pm}(f) & =\left\{(i, j) \in[\ell(w)]^{2}: i<j, X_{i}(f)=X_{j}^{ \pm}(f) \in\left\{x_{f}, x_{f}^{-1}\right\}\right\}, \\
\mathcal{N}_{S, \pi}^{0, \pm}(f) & =\left\{(i, j) \in \mathcal{N}_{w}^{ \pm}(f): \# \pi^{\prime}=\# \pi-1 \text { or } \# \mathcal{T}_{i, j}^{ \pm}(S)=\# S+1\right\}, \\
\mathcal{N}_{S, \pi}^{2, \pm}(f) & =\left\{(i, j) \in \mathcal{N}_{w}^{ \pm}(f): \# \pi^{\prime}=\# \pi \text { and } \# \mathcal{T}_{i, j}^{ \pm}(S)=\# S-1\right\}, \\
\mathcal{S}_{S, \pi}(f) & =\left\{(i, j) \in \mathcal{N}_{w}^{ \pm}(f): \# \pi^{\prime}=\# \pi\right\}
\end{aligned}
$$

and

$$
\mathcal{N}_{w}=\bigcup_{f=1}^{q} \mathcal{N}_{w}^{+}(f) \cup \mathcal{N}_{w}^{-}(f) .
$$

3.2.3. A differential system. Let us fix $q$ independent and identically distributed $\mathrm{U}(N)$ valued Brownian motions $\left(U_{1, s}\right)_{s \geq 0}, \ldots,\left(U_{q, s}\right)_{s \geq 0}$. We would like to show that Lemma 3 admits a natural generalization for cumulants instead of moments of traces in this 

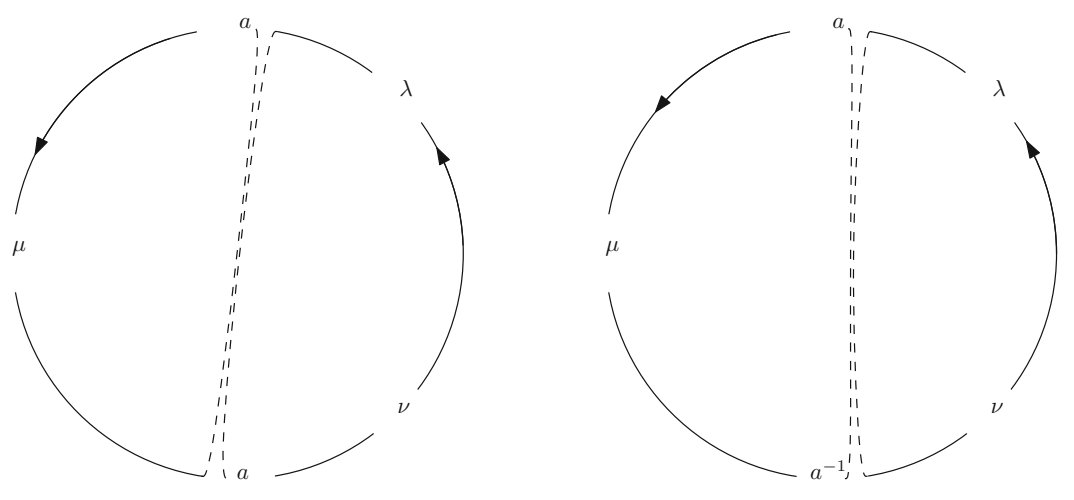

Fig. 1. Cut transformations

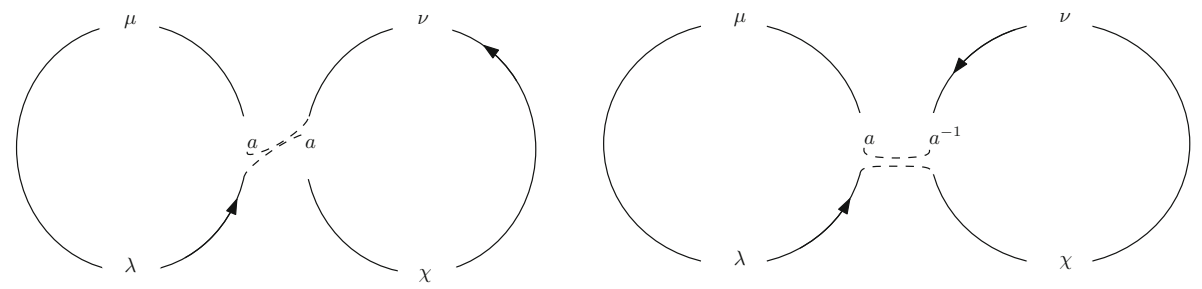

Fig. 2. Join transformations

multiple times setting. This will be achieved in Proposition 1. For any word decomposed as $w=x_{i_{1}}^{\epsilon_{1}} \ldots x_{i_{n}}^{\epsilon_{n}}$, with $\epsilon_{1}, \ldots, \epsilon_{n} \in\{-1,1\}$ and $U_{1}, \ldots, U_{q} \in \mathrm{U}(N)$, let us set

$$
w\left(U_{1}, \ldots, U_{q}\right)=U_{i_{1}}^{\epsilon_{1}} \ldots U_{i_{n}}^{\epsilon_{n}}
$$

and for any vector $t \in \mathbb{R}_{+}^{q}$,

$$
w_{t}^{N}=w\left(U_{1, t_{1}}, \ldots, U_{q, t_{q}}\right)
$$

For any partitioned word $(S, v) \in \mathcal{P} W_{q}$, with $S=\left(w_{1}, \ldots, w_{m}\right)$, we shall consider for any $t \in \mathbb{R}_{+}^{q}$,

$$
K_{t}(S, v)=C_{\# v}\left(\prod_{i \in A} \operatorname{Tr}\left(w_{i, t}^{N}\right), A \in v\right) .
$$

Lemma 6. For any $f \in[q]$ and any partitioned word $x=(S, v) \in \mathcal{P} W_{q}$,

$$
\frac{d}{d t_{f}} K_{t}(x)=-\frac{\bar{n}_{w}(f)}{2} K_{t}(x)-\frac{1}{N}\left(\sum K_{t}\left(\mathcal{T}_{i, j}^{+}(x)\right)-\sum K_{t}\left(\mathcal{T}_{i, j}^{-}(x)\right)\right),
$$

where the sums are over $(i, j)$ belonging respectively to $\mathcal{N}_{w(S)}^{+}(f)$ and $\mathcal{N}_{w(S)}^{-}(f)$.

Note that this Lemma could be proved differently than below, using the formulas of [40, Sect. 4.]. 
Remark 1. It is possible to adopt a more functional approach, closer to [13], describing the above operations in terms of the square of the formal operator of derivation with respect to one letter, given in [13], followed by a contraction. A possible framework could be the algebra of trace polynomials used in [9]. We leave here this question open.

Proof. Let us fix a tuple $\left(w_{1}, \ldots, w_{m}\right)$ of words, set $w=w_{1} \ldots w_{m}=x_{i_{1}}^{\epsilon_{1}} \ldots x_{i_{n}}^{\epsilon_{n}}$, with $\epsilon_{1}, \ldots, \epsilon_{n} \in\{-1,1\}$ and $\iota:[n] \rightarrow[m]$ the map induced by the decomposition of $w$ into $w_{1}, \ldots, w_{m}$. For any partition $v \in \mathcal{P}_{m}$, we denote by $\bar{v}^{0} \in \mathcal{P}_{n}$ the biggest partition such that $\iota\left(\bar{v}^{0}\right)=v$. For any $\sigma \in \mathfrak{S}_{n}$, let $S_{\sigma}$ be the tuple of words $x_{i_{c_{1}}}^{\epsilon_{c_{1}}} \ldots x_{i_{c_{k}}}$, such that $c_{1}<\ldots<c_{k}$ forms a cycle of $\sigma$, ordered by the position of their first letter in $w$. We denote $\mathcal{W}_{q}(w)=\left\{S_{\sigma}: \sigma \in \mathfrak{S}_{n}\right\}$ and fix an arbitrary injective map

$$
S \in \mathcal{W}_{q}(w) \mapsto \sigma_{S} \in \mathfrak{S}_{n}, \quad \text { such that } S_{\sigma_{S}}=S .
$$

Let us denote $\theta: M_{N}(\mathbb{C}) \rightarrow M_{N}(\mathbb{C}), M \mapsto M^{t}$, set for any integer $i \leq \ell(w)$, $\delta_{i}=\frac{1-\epsilon_{i}}{2}$, and define for any pair of distinct integers $i, j$ two operators $T_{i, j}^{+}$and $T_{i, j}^{-}$, acting on $\operatorname{End}\left(\left(\mathbb{C}^{N}\right)^{\otimes\{i, j\}}\right)$, by setting for any $M \in \operatorname{End}\left(\left(\mathbb{C}^{N}\right)^{\otimes\{i, j\}}\right)$,

$$
\begin{aligned}
T_{i, j}^{+}(M) & =M(i j), \\
T_{i, j}^{-}(M) & =\theta_{i}^{\delta_{i}} \circ \theta_{j}^{\delta_{j}}\left(\theta_{i}^{\delta_{i}} \circ \theta_{j}^{\delta_{j}}(M)\langle i j\rangle\right) .
\end{aligned}
$$

For any collection of words $S \in \mathcal{W}_{q}(w)$, any pair $1 \leq i<j \leq n$ and any $U_{1}, \ldots, U_{n} \in$ $\mathrm{U}(N)$ with $U_{i}^{\epsilon_{i}}=U_{j}^{\epsilon_{j}}$,

$$
\operatorname{Tr}_{\left(\mathbb{C}^{N}\right) \otimes n}\left(\sigma_{S} \bar{T}_{i, j}^{ \pm}\left(U_{1} \otimes \cdots \otimes U_{n}\right)\right)=\operatorname{Tr}_{\left(\mathbb{C}^{N}\right) \otimes n}\left(\sigma_{\mathcal{T}_{i, j}^{ \pm}(S)} U_{1} \otimes \cdots \otimes U_{n}\right) .
$$

It follows that if $U_{1}, \ldots, U_{n}$ are $\mathrm{U}(N)$-valued random variables with $U_{i}^{\epsilon_{i}}=U_{j}^{\epsilon_{j}}$, then for any partition $\pi \in \mathcal{P}_{n}$ such that $i$ and $j$ belong to the same block of $\pi$,

$$
\operatorname{Tr}_{\left(\mathbb{C}^{N}\right)^{\otimes n}}\left(\sigma_{S} C_{\pi, 1_{n}}\left(T_{i, j}^{ \pm}\left(\bigotimes_{1 \leq i \leq n} U_{i}\right)\right)\right)=\operatorname{Tr}_{\left(\mathbb{C}^{N}\right)^{\otimes n}}\left(\sigma_{\mathcal{T}_{i, j}^{ \pm}(S)} C_{\pi, 1_{n}}\left(\bigotimes_{1 \leq i \leq n} U_{i}\right)\right) .
$$

We shall now apply Lemma 4 to the tensors

$$
w_{t}^{\otimes A}=\bigotimes_{k \in A} U_{i_{k}, t_{i_{k}}}^{\epsilon_{k}}
$$

for any $A \subset[n]$, that we denote simply by $w_{t}^{\otimes}$, for $A=[n]$. For any $f \in[q]$, the latter yields

$$
\frac{d}{d t_{f}} \mathbb{E}\left[w_{t}^{\otimes A}\right]=-\frac{\bar{n}_{w, A}(f)}{2} \mathbb{E}\left[w_{t}^{\otimes A}\right]-\frac{1}{N}\left(\sum{\overline{T_{i, j}^{+}}}^{A}-\sum{\overline{T_{i, j}^{-}}}^{A}\right)\left(\mathbb{E}\left[w_{t}^{\otimes A}\right]\right),
$$

where the first and the second sums are over pairs $(i, j) \in A^{2}$ belonging respectively to $\mathcal{N}_{w}^{+}(f)$ and $\mathcal{N}_{w}^{-}(f)$. Consider now a partitioned word $(S, v)$ with $S \in \mathcal{W}_{q}(w)$. According to (8),

$$
K_{t}(S, v)=\operatorname{Tr}_{\left(\mathbb{C}^{N}\right)^{\otimes n}}\left(C_{\bar{v}^{0}, 1_{n}}\left[\sigma_{S} w_{t}^{\otimes}\right]\right)=\operatorname{Tr}_{\left(\mathbb{C}^{N}\right)^{\otimes n}}\left(\sigma_{S} C_{\bar{v}^{0}, 1_{n}}\left[w_{t}^{\otimes}\right]\right) .
$$




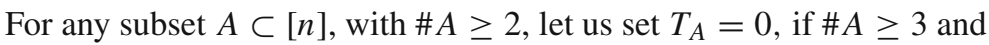

$$
T_{A}=\mp \frac{t_{f}}{N} T_{a, b}^{ \pm},
$$

if $A=\{a, b\}$, with $(a, b) \in \mathcal{N}_{w}^{ \pm}(f)$. Let us consider here elements of $M_{N}(\mathbb{C})$ as linear operators of $M_{N}(\mathbb{C})$ acting by left multiplication. The family of tensors

$$
\alpha_{A}=e^{\frac{1}{2} \sum_{1 \leq f \leq q} t_{f} \bar{n}_{w, A}(f)} \mathbb{E}\left[w_{t}^{\otimes A}\right] \in \operatorname{End}\left(V^{\otimes A}\right),
$$

for $A \subset[n]$, with $V=M_{N}(\mathbb{C})$, satisfies the condition of Lemma 5 with $\mu=\left\{\mathcal{N}_{w}^{ \pm}(f)\right.$ : $1 \leq f \leq q\}$. Hence, for any $f \in[q]$ and $\pi \in \mathcal{P}_{n}$,

$$
\frac{d}{d t_{f}} e^{\frac{1}{2} \sum_{1 \leq f \leq q} t_{f} \bar{n}_{w}(f)} C_{\pi, 1_{n}}\left[w_{t}^{\otimes}\right]=\sum_{(a, b) \in \mathcal{N}_{w}^{ \pm}(f)} C_{\pi \vee \overline{\{a, b\}}, 1_{n}}\left(t_{f}^{-1} \bar{T}_{\{a, b\}}\left(w_{t}^{\otimes}\right)\right) .
$$

For any pair $(a, b) \in \mathcal{N}_{w}$, if $p, q \in[m]$ are such that the $a^{\text {th }}$ and the $b^{\text {th }}$ letters of $w$ belong respectively to $w_{p}$ and $w_{q}$, then according to (17), the trace $-\epsilon_{a} \epsilon_{b} N \operatorname{Tr}_{\left(\mathbb{C}^{N}\right) \otimes n}\left(\sigma_{S} C_{\bar{v}^{0} \vee \overline{\{a, b\}}, 1_{n}}\left(t_{f}^{-1} \bar{T}_{\{a, b\}}\left(w_{t}^{\otimes}\right)\right)\right)$ is equal to

$$
\begin{aligned}
\operatorname{Tr}_{\left(\mathbb{C}^{N}\right)^{\otimes n}}\left(\sigma_{\mathcal{T}_{a, b}^{\epsilon_{i} \epsilon_{j}(S)}} C_{\bar{v}^{0} \vee \overline{\{a, b\}}, 1_{n}}\left(w_{t}^{\otimes}\right)\right) & =K_{t}\left(\mathcal{T}_{a, b}^{\epsilon_{i} \epsilon_{j}}(S), v \vee \overline{\{p, q\}}\right) \\
& =K_{t}\left(\mathcal{T}_{a, b}^{\epsilon_{i} \epsilon_{j}}(S, v)\right) .
\end{aligned}
$$

The two equations $(*)$ and $(* *)$ then imply the announced formula.

3.2.4. Scaling and asymptotic expansion of the cumulants. Let us now introduce a scaling of the above functions that matches the one of Sect. 3.1.1 and that yields a differential system with converging coefficients and initial conditions, as $N$ goes to infinity. For any partitioned word $(S, v) \in \mathcal{P} W_{q}$, with $S=\left(w_{1}, \ldots, w_{m}\right)$, the following quantity

$$
\varphi_{t, N}(S, v)=N^{2(\# v-1)-\ell(S)} C_{\# v}\left(\prod_{i \in A} \operatorname{Tr}\left(w_{i, t}\right), A \in v\right)
$$

satisfies these two conditions. Let us define two operators on $\mathbb{C}^{\mathcal{P} W_{q}}$, by setting for any function $\varphi \in \mathbb{C}^{\mathcal{P} W_{q}}$ and $(S, v) \in \mathcal{P} W_{q}, L_{f}(\varphi)(S, v)$ to be equal to

$$
-\frac{\bar{n}_{w(S)}(f)}{2} \varphi(S, v)+\sum_{(i, j) \in \mathcal{N}_{S, \pi}^{0,-}} \varphi\left(\mathcal{T}_{i, j}^{-}(x)\right)-\sum_{(i, j) \in \mathcal{N}_{S, \pi}^{0,+}} \varphi\left(\mathcal{T}_{i, j}^{+}(x)\right)
$$

and

$$
D_{f}(\varphi)(x)=\sum_{(i, j) \in \mathcal{N}_{S, v}^{2,-}} \varphi\left(\mathcal{T}_{i, j}^{-}(x)\right)-\sum_{(i, j) \in \mathcal{N}_{S, v}^{2,+}} \varphi\left(\mathcal{T}_{i, j}^{+}(x)\right)
$$


Proposition 1. For any $t \in \mathbb{R}_{+}^{q}, N \in \mathbb{N}^{*}$ and $(S, \pi) \in \mathcal{P} W_{q}$,

$$
\frac{d}{d t_{f}} \varphi_{t, N}(S, \pi)=\left(L_{f}+\frac{1}{N^{2}} D_{f}\right) \cdot \varphi_{t, N}(S, \pi)
$$

and $\varphi_{0, N}(S, \pi)=1$, if $\pi$ has one block and 0 otherwise. As $N \rightarrow \infty$, the sequence of functions $\varphi_{t, N}$ converges pointwise towards the unique function $\varphi_{t}$, such that for any $t \in \mathbb{R}_{+}^{q}$ and $(S, \pi) \in \mathcal{P} W_{q}$,

$$
\frac{d}{d t_{f}} \varphi_{t}(S, \pi)=L_{f} \cdot \varphi_{t}(S, \pi)
$$

and $\varphi_{0}=\varphi_{0,1}$.

Proof. It is a direct consequence of Lemma 6.

Corollary 1. There exists a sequence offunctions $\left(\psi_{t, g}\right)_{g \geq 1}$ on $\mathcal{P} W_{q}$ such for any $(S, \pi)$, the power series with coefficients $\left(\psi_{t, g}(S, \pi)\right)_{g \geq 1}$ has a positive radius of convergence and for $N$ large enough,

$$
\varphi_{t, N}(S, \pi)=\varphi_{t}(S, \pi)+\sum_{g \geq 1} N^{-2 g} \psi_{t, g}(S, \pi) .
$$

Proof. For any fixed $n \in \mathbb{N}^{*}$, the operators $L_{f}, D_{f}$ preserve the finite dimensional space of functions supported on $\left\{x \in \mathcal{P} W_{q}: \ell(x) \leq n\right\}$. The above expansion follows then easily from Proposition 1.

In particular, for any $n_{1}, \ldots, n_{m} \in \mathbb{Z}, N^{m-2} C_{m}\left(\operatorname{Tr}\left(U_{t}^{n_{1}}\right), \ldots, \operatorname{Tr}\left(U_{t}^{n_{m}}\right)\right)$ admits a limit as $N \rightarrow \infty$. Together with the property of independence, stationarity and invariance by unitary adjunction of multiplicative increments of the process $\left(U_{t}\right)_{t \geq 0}$ and the notion of higher order freeness developed in [14], this fact alone implies that for any $(S, \pi) \in$ $\mathcal{P} W_{q}, \varphi_{t, N}(S, \pi)$ admits a limit as $N \rightarrow \infty$. Nonetheless, this result does not give easily an expansion in $N$.

\section{Two Estimates on the Cumulants}

The proof of Theorem 1 relies on two estimates on the above cumulants. The first one shall allow to extend them to broader class of words as we will see in Sect. 6. It is nonetheless too loose to obtain a positive radius of convergence as stated in Theorem 1. The second type of estimates gives a much sharper bound and is the key ingredient to address this question.

4.1. All-order bounds. For any $t \in \mathbb{R}_{+}^{q}$, let us define a scalar product $\langle\cdot, \cdot\rangle_{t}$ on $\mathbb{R}^{q}$, by setting for any $a, b \in \mathbb{R}^{q}$,

$$
\langle a, b\rangle_{t}=\sum_{f=1}^{q} a_{f} b_{f} t_{t} .
$$

Let us recall the notations of Sect. 3.2.1 and set for any word $w \in W_{q}$,

$$
A_{t}(w)=\left\langle\bar{n}_{w}, \bar{n}_{w}\right\rangle_{t}
$$


For any $(S, \pi) \in \mathcal{P} W_{q}$ and $N \in \mathbb{N}^{*}$, we define inductively a sequence by setting $\psi_{t, 0, N}(S, \pi)=\varphi_{t, N}(S, \pi)$ and for any $g \in \mathbb{N}$,

$$
\psi_{t, g+1, N}(S, \pi)=N^{2}\left(\psi_{t, g, N}(S, \pi)-\psi_{t, g}(S, \pi)\right) .
$$

Lemma 7. For any words $w_{1}, \ldots, w_{m} \in W_{q}, N \in \mathbb{N}^{*}, t \in \mathbb{R}_{+}^{q}, \lambda \in[0,1]$ and $k \in \mathbb{N}$,

$$
\left|\frac{d^{k}}{d \lambda^{k}} \varphi_{\lambda t, N}\left(w_{1}, \ldots, w_{m}\right)\right| \leq 2^{-k} A_{t}\left(w_{1} \ldots w_{m}\right)^{k} e^{\frac{A_{t}\left(w_{1} \ldots w_{m}\right)}{2}}
$$

and

$$
\left|\psi_{t, k, N}\left(w_{1}, \ldots, w_{m}\right)\right| \leq 2^{-k} A_{t}\left(w_{1} \ldots w_{m}\right)^{k} e^{\frac{A_{t}\left(w_{1} \ldots w_{m}\right)}{2}} .
$$

Besides, $A_{t}\left(w_{1} \ldots w_{m}\right) \leq m \sum_{i=1}^{m} A_{t}\left(w_{i}\right)$.

Proof. For every integer $p \in \mathbb{N}$ and any matrix $M \in M_{p}(\mathbb{C})$, let us set $\|M\|=$ $\max _{i \in[p]} \sum_{j=1}^{p}\left|M_{i, j}\right|$. Recall that $\|\cdot\|$ is a sub-multiplicative norm on $M_{p}(\mathbb{C})$, such that for any matrix $M \in M_{p}(\mathbb{C})$ and $v \in \mathbb{C}^{p}, \max _{i \in[p]}\left|(M v)_{i}\right| \leq\|M\| \max _{i \in[p]}\left|v_{i}\right|$. Let us fix a word $w \in W_{q}$ and denote by $B_{w}$ the set of partitioned words $(S, \pi) \in \mathcal{P} W_{q}$ with $n_{w(S)}^{ \pm}=n_{w}^{ \pm}$. Note that $B_{w}$ is stable by the operations appearing in (23) and (24), so that for any $f \in[q]$, the two operators $L_{f}$ and $D_{f}$ preserve the finite dimensional space $\mathcal{F}_{w}$ of functions on $\mathcal{P} W_{q}$, with support in $B_{w}$. For any $F \in \operatorname{End}\left(\mathcal{F}_{w}\right)$, let us set

$$
\|F\|=\max _{y \in B_{w}} \sum_{x \in B_{w}} F\left(\delta_{x}\right)(y) .
$$

For any $\left(S^{\prime}, \pi^{\prime}\right) \in B_{w}$, there are at most $\frac{\bar{n}_{w}(f)\left(\bar{n}_{w}(f)-1\right)}{2}$ elements $(S, \pi)$ of $B_{w}$ such that $\left(S^{\prime}, \pi^{\prime}\right)$ is obtained from $(S, \pi)$ by a transformation of the form $\mathcal{T}_{i, j}^{ \pm}$with $(i, j) \in \mathcal{N}_{w(S)}$. It follows that the restriction of $L_{f}$ and $D_{f}$ to $\mathcal{F}_{w}$ satisfy the following inequality

$$
\max \left\{\left\|L_{f_{\mid \mathcal{F}_{w}}}\right\|,\left\|D_{f_{\mid \mathcal{F}}}\right\|,\left\|L_{f_{\mid \mathcal{F}_{w}}}+\frac{1}{N^{2}} D_{f_{\mid \mathcal{F}_{w}}}\right\|\right\} \leq \frac{\bar{n}_{w}(f)^{2}}{2}
$$

Let us set

$$
L=\sum_{f=1}^{q} t_{f} L_{f_{\mid \mathcal{F}_{w}}} \quad \text { and } \quad D=\sum_{f=1}^{q} t_{f} D_{f_{\mid \mathcal{F}_{w}}} .
$$

Then, according to Proposition 1,

$$
\varphi_{t, N \mid B_{w}}=e^{L+\frac{1}{N^{2}} D}\left(\varphi_{0 \mid B_{w}}\right)
$$

and

$$
\varphi_{t \mid B_{w}}=e^{L}\left(\varphi_{0 \mid B_{w}}\right) .
$$

Let us recall that for any matrices $A, B \in M_{p}(\mathbb{C})$,

$$
e^{A+B}-e^{A}=\int_{0}^{1} e^{s(A+B)} B e^{(1-s) A} d s .
$$


Using iteratively this formula together with the former two equations yields that for any $g \geq 1$,

$$
\psi_{t, g, N_{\mid B_{w}}}=\int_{0<s_{1}<s_{2}<\ldots<s_{g}<1} e^{s_{1}\left(L+\frac{1}{N^{2}} D\right)} D e^{\left(s_{2}-s_{1}\right) L} D \ldots D e^{\left(1-s_{g}\right) L} d s\left(\varphi_{0 \mid B_{w}}\right) .
$$

Therefore,

$$
\begin{aligned}
\max _{(S, \pi) \in B_{w}}\left\{g !\left|\psi_{t, g, N}(S, \pi)\right|\right\} & \leq\|D\|^{g} e^{\max \left\{\left\|L+\frac{1}{N^{2}} D\right\|,\|L\|\right\}} \max _{(S, \pi) \in B_{w}}\left\{\varphi_{0}(S, \pi)\right\} \\
& \leq 2^{-g}\left(\sum_{f=1}^{q} t_{f} \bar{n}_{w}^{2}\right)^{g} e^{\frac{1}{2} \sum_{f=1}^{q} t_{f} \bar{n}_{w}^{2}}=2^{-g} A_{t}(w)^{g} e^{\frac{A_{t}(w)}{2}} .
\end{aligned}
$$

Besides, for all $\lambda \in[0,1]$ and $r \in \mathbb{N}$,

$$
\frac{d^{r}}{d \lambda^{r}} \varphi_{\lambda t, N \mid B_{w}}=\left(L+\frac{1}{N^{2}} D\right)^{r} e^{\lambda\left(L+\frac{1}{N^{2}} D\right)}\left(\varphi_{0 \mid B_{w}}\right),
$$

so that the left-hand-side is uniformly bounded by $2^{-r} A_{t}(w)^{r} e^{\frac{A_{t}(w)}{2}}$ on $B_{w}$. Besides, for any words $w_{1} \ldots w_{m} \in W_{q}$, if $w=w_{1} \ldots w_{m}$, then $\bar{n}_{w}=\bar{n}_{w_{1}}+\cdots+\bar{n}_{w_{m}}$ and $A_{t}(w)=\left\|\bar{n}_{w}\right\|_{t}^{2}$, the last point follows.

Note that these first estimates are very loose. For example, for $w=x_{1}^{n}$, the lemma shows that $\left|\varphi_{t}(w)\right| \leq e^{t_{1} n^{2}}$, when we have ${ }^{4}$ the simple bound $\left|\varphi_{t}(w)\right|=\left|\int_{\mathbb{U}} \omega^{n} \mu_{t_{1}}(d \omega)\right| \leq 1$. Furthermore, for this same word, it yields for any $m \in \mathbb{N}^{*},\left|N^{m-2} C_{m}\left(\operatorname{Tr}\left(U_{t_{1}}^{n}\right)\right)\right| \leq$ $e^{t_{1} n^{2} m^{2}}$, so that the exponential power series of the sequence on the right-hand-side diverges.

4.2. Sharper bounds for the first and second orders. For any positive integer $m$, let us denote by $\mathcal{C}_{m}$ the set of Cayley trees on $m$ vertices. For any $w_{1}, \ldots, w_{m} \in W_{q}, \mathfrak{T} \in \mathcal{C}_{m}$, we set

$$
\mathfrak{T}_{t}\left(w_{1}, \ldots, w_{m}\right)=\prod_{\{i, j\} \text { edge of } \mathfrak{T}}\left\langle\bar{n}_{w_{i}}, \bar{n}_{w_{j}}\right\rangle_{t}
$$

and

$$
\tilde{\mathfrak{T}}_{t}\left(w_{1}, \ldots, w_{m}\right)=\prod_{\{i, j\} \text { edge of } \mathfrak{T}}\left\langle n_{w_{i}}, n_{w_{j}}\right\rangle_{t},
$$

if $m \geq 2$, and $\mathfrak{T}_{t}\left(w_{1}\right)=\tilde{\mathfrak{T}}_{t}\left(w_{1}\right)=1$, otherwise.

Proposition 2. For any words $w_{1}, \ldots, w_{m} \in W_{q}$ and any $N \in \mathbb{N}^{*}$,

$$
\left|\varphi_{t, N}\left(w_{1}, \ldots, w_{m}\right)\right| \leq \sum_{\mathfrak{T} \in \mathcal{C}_{m}} \mathfrak{T}_{t}\left(w_{1}, \ldots, w_{m}\right)
$$

\footnotetext{
4 The right decay of this moment sequence is at least polynomial, as the measure $\mu_{t_{1}}$ is absolutely continuous with respect to the Lebesgue measure, with a density that is Hölder continuous. The regularity of the density does depend on $t_{1}$, there are three regimes: $t_{1}<4, t_{1}=4$ and $t_{1}>4$, see Remark 6.8. of [34].
} 
and for $m \geq 2$,

$$
\varphi_{t, N}\left(w_{1}, \ldots, w_{m}\right)=\sum_{\mathfrak{T} \in \mathcal{C}_{m}} \tilde{\mathfrak{T}}_{t}\left(w_{1}, \ldots, w_{m}\right)+R_{t, N}\left(w_{1}, \ldots, w_{m}\right),
$$

with $\left|R_{t, N}\left(w_{1}, \ldots, w_{m}\right)\right| \leq\left(\frac{m}{2}\right)^{m}\left(A_{t}\left(w_{1}\right)+\cdots+A_{t}\left(w_{m}\right)\right)^{m} e^{\frac{m}{2}\left(A_{t}\left(w_{1}\right)+\cdots+A_{t}\left(w_{m}\right)\right)}$, whereas

$$
\varphi_{t, N}\left(w_{1}\right)=1-\frac{1}{2}\left\|n_{w}\right\|_{t}^{2}+R_{t, N}\left(w_{1}\right)
$$

with $\left|R_{t, N}\left(w_{1}\right)\right| \leq A_{t}\left(w_{1}\right)^{2} e^{\frac{A_{t}\left(w_{1}\right)}{2}}$,

The second estimate shows that the first one is optimal for tuples of words $w \in W_{q}$, such that $\bar{n}_{w}=n_{w}$. The idea of the following proof is to get formulas for the considered cumulants not in terms of partitioned words, as we did in Lemma 7, but rather in terms of their "Schur-Weyl dual", that is here, tensors of unitary matrices.

Proof. Let us consider $q$ independent Brownian motions $\left(U_{1, t}\right)_{t \geq 0}, \ldots,\left(U_{q, t}\right)_{t \geq 0}$, on $\mathrm{U}(N)$, fix a tuple of $m$ words $S_{0}=\left(w_{1}, \ldots, w_{m}\right) \in W_{q}^{m}$, set $w=w_{1} \ldots w_{m}=$ $x_{i_{1}}^{\epsilon_{1}} \ldots x_{i_{n}}^{\epsilon_{n}}, \sigma_{0}=\left(1 \ldots \ell\left(w_{1}\right)\right) \times \cdots \times\left(1 \ldots \ell\left(w_{m}\right)\right)$ and $\pi_{0} \in \mathcal{P}_{n}$ the set of its orbits ordered by their smallest element and recall Definition (18). Let us further use the same notations as in the first paragraph of proof of Lemma 6 . According our choice of scaling and (8),

$$
\varphi_{t, N}\left(w_{1}, \ldots, w_{m}\right)=N^{m-2} \operatorname{Tr}_{\left(\mathbb{C}^{N}\right) \otimes n}\left(\sigma_{0} C_{\pi_{0}, 1_{m}}\left(w_{t}^{\otimes}\right)\right) .
$$

Let us remind that the family of tensors (21) satisfies the condition of Lemma 5, with $\left(T_{A}\right)_{A \in \mathfrak{B}_{n}}$ given in (20). We shall denote here $T_{\{p, q\}}$ by $T_{p, q}$. Let $\Psi_{m}$ be the set of strictly increasing sequences $\left(v_{i}\right)_{i=1}^{m} \in \mathcal{P}_{m}^{m}$, with $v_{1}=0_{m}$ and $v_{m}=1_{m}$. Each sequence of pairs $\left(p_{l}, q_{l}\right)_{1 \leq l \leq m-1} \in \mathcal{N}_{w}^{k}$, with $\vee_{l=1}^{m-1} \overline{\left\{p_{l}, q_{l}\right\}} \vee \pi_{0}=1_{n}$, induces an element of $\Psi_{m}$ and any element of $\Psi_{m}$ can be obtained in this way. For any $v \in \Psi_{m}$, we set $\Upsilon(v)=\left\{\left(p_{k}, q_{k}\right)_{1 \leq k \leq m-1}: \forall k \in[m-1], p_{k}<q_{k}, \vee_{i=1}^{k}\left\{p_{i}, q_{i}\right\} \vee \pi_{0}=v_{k}\right\}$. According to (9), $e^{\frac{1}{2} \sum_{1 \leq f \leq q} t_{f} \bar{n}_{w}(f)} C_{\pi_{0}, 1_{m}}\left(w_{t}^{\otimes}\right)$ equals

$$
\beta_{\pi_{0}, 1_{m}}(\alpha)=\sum_{\nu \in \Psi_{m}} \sum_{\left(p_{k}, q_{k}\right)_{k} \in \Upsilon(\nu)} \int_{\Delta^{m-1}} \alpha_{\pi}^{s_{0}} \bar{T}_{p_{1}, q_{1}} \alpha_{\nu_{1}}^{s_{1}} \ldots \bar{T}_{p_{m-1}, q_{m-1}} \alpha_{1_{m}}^{s_{m}} d s .
$$

Let us now rewrite the right-hand-side in terms of unitary matrices thanks to the Definitions (6) and (21). For any partition $\mu \in \mathcal{P}_{m}$, let us denote by $\mu:[n] \rightarrow \mu$ the natural quotient map and introduce a collection $\left(\left(U_{t, b, f}\right)_{t \geq 0}\right)_{b \in \mu, 1 \leq f \leq q}$ of $q \# \mu$ independent $\mathrm{U}(N)$-Brownian motions. Then, let $\left(U^{\mu}\right)_{\mu \in \mathcal{P}_{n}}$ be a collection of independent random variables such that for each $\mu \in \mathcal{P}_{n}$,

$$
\left(U_{t_{k}, k}^{\mu}\right)_{t \in \mathbb{R}_{+}^{n}, 1 \leq k \leq n} \stackrel{(\text { law) }}{=}\left(U_{t_{k}, \underline{\mu}(k), i_{k}}^{\epsilon_{k}}\right)_{t \in \mathbb{R}_{+}^{n}, 1 \leq k \leq n} .
$$

For any $v \in \operatorname{End}\left(M_{N}(\mathbb{C})^{\otimes n}\right)$, let us denote by $\mathcal{L}(v)$ the endomorphism of $\operatorname{End}\left(M_{N}(\mathbb{C})^{\otimes n}\right)$, of left multiplication by $v$. With these notations, (29) yields that the cumulant $C_{\pi_{0}, 1_{m}}\left(w_{t}^{\otimes}\right)$ equals 


$$
\sum_{\nu \in \Psi_{m}} \sum_{\left(p_{k}, q_{k}\right)_{k} \in \Upsilon(\nu)} \int_{\Delta^{m-1}} \mathbb{E}\left[\mathcal{L}\left(\bigotimes_{k=1}^{n} U_{s_{1} t_{i_{k}}, k}^{\nu_{1}}\right) \bar{T}_{p_{1}, q_{1}} \ldots \bar{T}_{p_{m}, q_{m}} \mathcal{L}\left(\bigotimes_{k=1}^{n} U_{s_{m} t_{i_{k}}, k}^{v_{m}}\right)\right] d s .
$$

Notice that for every matrices $U_{1}, \ldots, U_{n} \in \mathrm{U}(N)$ and any pair $(a, b) \in \mathcal{N}_{w}^{ \pm}$, such that $U_{a}=U_{b}, \mathcal{L}\left(\bigotimes_{k \in[n]} U_{k}\right)$ commutes with $\bar{T}_{a, b}$. Therefore, if we set for any sequence $v \in \Psi_{m}, s \in \Delta^{m-1}$ and $1 \leq k \leq n$,

$$
V_{s, k}^{v}=U_{s_{1} t_{i}, k}^{\nu_{1}} \ldots U_{s_{m} t_{i}, k}^{v_{m}} \in \mathrm{U}(N),
$$

then, $\operatorname{Tr}_{\left(\mathbb{C}^{N}\right)^{\otimes n}}\left(\sigma_{0} C_{\pi, 1_{m}}\left(w_{t}^{\otimes}\right)\right)$ equals

$$
\sum_{\nu \in \Psi_{m}} \sum_{\left(p_{k}, q_{k}\right)_{k} \in \Upsilon(v)} \int_{\Delta^{m-1}} \mathbb{E}\left[\operatorname{Tr}\left(\sigma_{0} \bar{T}_{p_{m-1}, q_{m-1}} \ldots \bar{T}_{p_{1}, q_{1}}\left(\otimes_{k \in[n]} V_{s, k}^{v}\right)\right)\right] d s .
$$

For each $v \in \Psi_{m}, \gamma=\left(p_{k}, q_{k}\right)_{k} \in \Upsilon(v)$, there exists a word $w_{\gamma}$ such that $\mathcal{T}_{\tilde{p}_{m-1}, \tilde{q}_{m-1}}^{\epsilon_{m-1}} \circ$ $\cdots \circ \mathcal{T}_{\tilde{p}_{2}, \tilde{q}_{2}}^{\epsilon_{2}} \circ \mathcal{T}_{p_{1}, q_{1}}^{\epsilon_{1}}\left(S_{0}, 0_{m}\right)=\left(w_{\gamma}, 1_{1}\right)$, where for any $k \in[m-1], \epsilon_{k}=\epsilon_{p_{k}} \epsilon_{q_{k}}$ and for $2 \leq k<m, \tilde{p}_{k}, \tilde{q}_{k}$ are the new positions of the $p_{k}^{\text {th }}$ and $q_{k}^{\text {th }}$ letters of $S_{0}$ in $\mathcal{T}_{\tilde{p}_{k-1}, \tilde{q}_{m-1}}^{\epsilon_{k-1}} \circ \cdots \circ \mathcal{T}_{p_{1}, q_{1}}^{\epsilon_{1}}\left(S_{0}\right)$. If $t_{\gamma}=\prod_{k=1}^{m-1}\left(-\epsilon_{i_{p_{k}}} \epsilon_{i_{q_{k}}}\right) t_{i_{p_{k}}}$, then, according to (17), for any tensor $v \in M_{N}(\mathbb{C})^{\otimes n}$,

$$
\operatorname{Tr}\left(\sigma_{0} \bar{T}_{p_{m-1}, q_{m-1}} \ldots \bar{T}_{p_{1}, q_{1}}(v)\right)=t_{\gamma} N^{1-m} \operatorname{Tr}\left(\sigma_{w_{\gamma}} v\right),
$$

where $\sigma_{w_{\gamma}}$ is defined as (14). It remains now to unfold the above notations to get

$$
\left.\varphi_{t, N}\left(w_{1}, \ldots, w_{m}\right)=\sum_{\nu \in \Psi_{m}} \sum_{\gamma \in \Upsilon(\nu)} t_{\gamma} \int_{\Delta^{m-1}} \mathbb{E}\left[N^{-1} \operatorname{Tr}\left(w_{\gamma}\left(V_{s, 1}^{v}, \ldots, V_{s, n}^{v}\right)\right)\right)\right] d s .
$$

To conclude, note that the normalized trace in the integrand of the right-hand-side are bounded by 1 . Let $\Gamma: \Psi_{m} \rightarrow \mathcal{C}_{m}$ be the $(m-1)$ !-to-one map that sends every sequence $v=\left(\vee_{l=1}^{k} \overline{\left\{i_{l}, j_{l}\right\}}\right)_{1 \leq k<m}$ to the Cayley tree with edges $\left(\left\{i_{k}, j_{k}\right\}\right)_{1 \leq k<m}$. Then, for every $v \in \Psi_{m}, \sum_{\gamma \in \Upsilon(v)}\left|t_{\gamma}\right|=\Gamma(v)\left(w_{1}, \ldots, w_{m}\right)$ and the first bound of the statement follows. Another consequence of (31), is that, in the Taylor expansion of $\varphi_{\cdot, N}\left(w_{1}, \ldots, w_{m}\right)$ around $0 \in \mathbb{R}_{+}^{q}$, the terms of degree less than $m-2$ vanish, whereas, the sum of terms of degree $m-1$ is exactly $\sum_{\nu \in \Psi_{m}} \sum_{\gamma \in \Upsilon(v)} \frac{t_{\gamma}}{(m-1) !}$. But, for any $\mathfrak{T} \in \mathcal{C}_{m}$ and $v \in \Gamma^{-1}(\mathfrak{T})$, $\sum_{\gamma \in \Upsilon(v)} t_{\gamma}=\tilde{\mathfrak{T}}_{t}\left(w_{1}, \ldots, w_{m}\right)$. Hence, applying Proposition 7 yields the second estimate. A similar argument applies for $\varphi_{t, N}\left(w_{1}\right)$.

Remark 2. When $N=1,\left(w_{k, t}\right)_{1 \leq k \leq m}$ has the same law as $\left(\exp \left(i Z_{k}\right)\right)_{1 \leq k \leq m}$, where $\left(Z_{k}\right)_{1 \leq k \leq m}$ is a Gaussian vector of covariance matrix $\left(\left\langle n_{w_{p}}, n_{w_{q}}\right\rangle_{t}\right)_{1 \leq p, q \leq m}$. It follows from the definition that

$$
\varphi_{t, 1}(w)=e^{-\frac{1}{2}\left\|n_{w}\right\|_{t}^{2}},
$$

whereas for $w \in W_{q}^{m}$, the Leonov Shiryaev formula (5), shows that for any $m \geq 2$,

$$
\varphi_{t, 1}\left(w_{1}, \ldots, w_{m}\right)=\sum_{\mathfrak{T} \in \mathcal{C}_{m}} \tilde{\mathfrak{T}}\left(w_{1}, \ldots, w_{m}\right) e^{-\frac{1}{2}\left\|n_{w_{1}}+\ldots+n_{w_{m}}\right\|_{t}^{2}} .
$$


Remark 3. Formula (31) implies that any cumulant $\varphi_{t, N}\left(w_{1}, \ldots, w_{m}\right)$ is expressed in terms of the non-commutative distribution of the unitary Brownian motion.

For any $t \in \mathbb{R}_{+}$, let us set

$$
\lambda_{t}=(t+1+\sqrt{t(t+2)}) e^{\frac{1}{2} \sqrt{t(t+2)}}
$$

and for any word $w \in W_{q}$ and $t \in \mathbb{R}_{+}^{q}$,

$$
\lambda_{t}(w)=\prod_{f=1}^{q} \lambda_{t_{i}}^{\bar{n}_{w}(f)} .
$$

Lemma 8. For any sequence $S=\left(w_{1}, \ldots, w_{m}\right)$ of words in $W_{q}$ with $m \geq 2$ and any $N \in \mathbb{N}^{*}$,

$$
\left|\psi_{t, 1, N}(S)\right| \leq 2^{m-1} m^{2} \prod_{i=1}^{m} \lambda_{t}\left(w_{i}\right) \max _{1 \leq i \leq m}\left\|\bar{n}_{w_{i}}\right\|_{t}^{2} \sum_{\mathfrak{T} \in \mathcal{C}_{m}} \mathfrak{T}(S) .
$$

Proof. Let us fix $S_{0} \in W_{q}^{m}, B_{0}$ the set of partitioned word obtained from $x_{0}=\left(S, 0_{m}\right)$ by a sequence of cut and join transformations and set for any $\eta \in \mathcal{P}_{m}, B_{\eta}=\left\{\left(S^{\prime}, \eta\right)\right.$ : $\left.\left(S^{\prime}, \eta\right) \in B_{0}\right\}$. For any $N \in \mathbb{N}^{*}$, according to Proposition 1 and Duhamel's formula (26), if $L$ and $D$ are defined as in (25),

$$
\psi_{t, 1, N}\left(S_{0}\right)=-\int_{0}^{1} e^{(1-s) L} D e^{s\left(L+N^{-2} D\right)}\left(\varphi_{0}\right)\left(x_{0}\right) d s .
$$

Setting for any $s \in[0,1]$ and $x, y \in B_{0}, Q_{s}(x, y)=e^{s L} D\left(\delta_{y}\right)(x)$, yields

$$
\begin{aligned}
-\psi_{t, 1, N}\left(S_{0}\right) & =\sum_{y \in B_{0}} \int_{0}^{1} Q_{1-s}\left(x_{0}, y\right) e^{s\left(L+N^{-2} D\right)}\left(\varphi_{0}\right)(y) d s \\
& =\sum_{\eta \in \mathcal{P}_{m}} \sum_{y \in B_{\eta}} \int_{0}^{1} \varphi_{s t, N}(y) Q_{1-s}\left(x_{0}, y\right) d s .
\end{aligned}
$$

Let us fix $\eta \in \mathcal{P}_{m}$ and consider the space of strictly increasing sequences $\Psi^{\eta}=$ $\left\{\left(\vee_{i=1}^{k} \overline{\left\{p_{i}, q_{i}\right\}}\right)_{0 \leq k \leq m-\# \eta} \in \mathcal{P}_{m}^{m-\# \eta+1}: \vee_{i=1}^{m-\# \eta} \overline{\left\{p_{i}, q_{i}\right\}}=\eta\right\}$ and $\Psi_{\eta}=\left\{\left(\vee_{i=1}^{k} \overline{\left\{p_{i}, q_{i}\right\}} \vee\right.\right.$ $\left.\eta)_{0 \leq k \leq \# \eta-1} \in \mathcal{P}_{m}^{\# \eta}: \vee_{i=1}^{\# \eta} \overline{\left\{p_{i}, q_{i}\right\}} \vee \eta=1_{m}\right\}$. For any $t \in \mathbb{R}_{+}^{q}$ and any increasing sequence $v \in \mathcal{P}_{m}^{l}$, induced by a sequence of pairs of integers $\left(p_{i}, q_{i}\right)_{1 \leq i \leq l}$ of $[m]$, let us set

$$
v_{t}\left(S_{0}\right)=\prod_{k=1}^{l}\left\langle\bar{n}_{w_{p_{k}}}, \bar{n}_{w_{q_{k}}}\right\rangle_{t} .
$$

For any $\pi \in \mathcal{P}_{m}$ and any linear operator $A$ on $\mathbb{C}^{B_{0}}$, let us introduce another operator $A_{\pi}$ by setting for any $\varphi \in \mathbb{C}^{B_{0}}$ and $x \in B_{0}, A_{\pi}(\varphi)(x)=\sum_{y \in B_{\pi}} \varphi(y) A\left(\delta_{y}\right)(x)$. On one hand, for any $y \in B_{\eta}$, a slight modification of the proof of Proposition 2 yields that for any $t \in \mathbb{R}_{+}^{q}$, 


$$
\left|\varphi_{t, N}(y)\right| \leq \frac{1}{(\# \eta-1) !} \sum_{\nu \in \Psi_{\eta}} v_{t}\left(S_{0}\right) .
$$

On the other hand, the same argument as in Lemma 5 yields that $\sum_{y \in B_{\eta}}\left|Q_{1}\left(x_{0}, y\right)\right|$ is bounded by

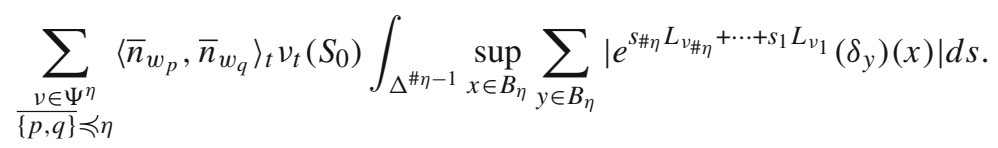

To conclude, we shall expand the exponential in the right-hand-side and use then triangular inequality. For each $f \in[q]$, let us define an operator $\tilde{L}_{f}$ on $\mathbb{C}^{\mathcal{P} W_{q}}$, by setting for all $\varphi \in \mathbb{C}^{\mathcal{P} W_{q}}$ and $x=(S, \pi) \in B_{0}$,

$$
\tilde{L}_{f}(\varphi)(x)=\sum_{(a, b) \in \mathcal{N}_{S, \pi}^{+, 0}(f)} \varphi\left(\mathcal{T}_{a, b}^{+}(x)\right)+\sum_{(a, b) \in \mathcal{N}_{S, \pi}^{-, 0}(f)} \varphi\left(\mathcal{T}_{a, b}^{-}(x)\right) .
$$

For any $x \in B_{0}, v \in \Psi^{\eta}$ and $s \in \Delta^{\# \eta-1}$,

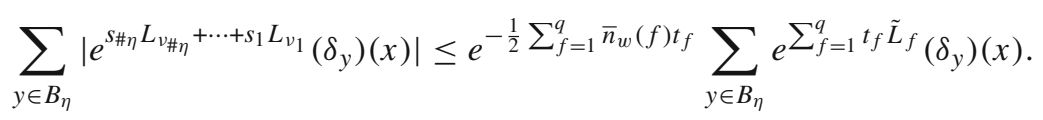

For any tuple $S$ of words in $W_{q}$, let us denote, for each $f \in[q]$, by $w_{f}(S) \in W_{q}$, the word obtained from $w(S)$ by deleting the letters $x_{f^{\prime}}$ and $x_{f^{\prime}}^{-1}$, for $f^{\prime} \neq f$. Recall that for any $x=(S, \pi) \in \mathcal{P} W_{q}, \varphi_{0}(x)=1$, if $\# \pi=1$, and 0 , otherwise. Then, for any $x=(S, \pi) \in B_{v}$,

$$
\sum_{y \in B_{\eta}} e^{\sum_{f=1}^{q} t_{f} \tilde{L}_{f}}\left(\delta_{y}\right)(x) \leq \prod_{f=1}^{q} e^{t_{f} \tilde{L}_{f}}\left(\varphi_{0}\right)\left(w_{f}(S), 1_{\bar{n}_{w}(f)}\right) .
$$

Let us denote

$$
\Lambda_{t}(w)=e^{-\frac{1}{2} \sum_{f=1}^{q} t_{f} \bar{n}_{w}(f)} \sup _{(S, \pi) \in B_{0}} \prod_{f=1}^{q} e^{t_{f} \tilde{L}_{f}}\left(\varphi_{0}\right)\left(w_{f}(S), 1_{\bar{n}_{w}(f)}\right) .
$$

Gathering the equality $(\diamond)$ with the last four inequalities yields that the quantity $e^{\sum_{f=1}^{q} t_{f} \bar{n}_{w}(f)} \psi_{t, 1, N}\left(S_{0}, 0_{m}\right)$ is bounded by

$$
\begin{gathered}
\sum_{\substack{\eta \in \mathcal{P}_{m} \\
v^{1} \in \Psi^{\eta}, v^{2} \in \Psi_{\eta}}} \frac{v_{t}^{1}\left(S_{0}\right) v_{t}^{2}\left(S_{0}\right)}{(\# \eta-1) !(m-\# \eta) !} \Lambda_{t}(w) \sup _{1 \leq i \leq m}\left\|\bar{n}_{w_{i}}\right\|_{t}^{2} \\
\leq \Lambda_{t}(w) \sup _{1 \leq i \leq m}\left\|\bar{n}_{w_{i}}\right\|_{t}^{2} \sum_{v \in \Psi_{m-1}} \sum_{k=1}^{m} \frac{v_{t}\left(S_{0}\right)}{(k-1) !(m-k) !} \\
\leq 2^{m-1} \Lambda_{t}(w) \sup _{1 \leq i \leq m}\left\|\bar{n}_{w_{i}}\right\|_{t}^{2} \sum_{\mathfrak{T} \in \mathcal{C}_{m}} \mathfrak{T}\left(S_{0}\right) .
\end{gathered}
$$

Then, the following lemma implies the announced bound. 
Lemma 9. For any word $w \in W_{q}, t \in \mathbb{R}_{+}^{q}$,

$$
\Lambda_{t}(w) \leq \lambda_{t}(w)
$$

where both terms are given by (34) and (32).

Proof. For any $w=x^{\epsilon_{1}} \ldots x^{\epsilon_{n}} \in W_{1}$, let us define $\mathcal{I}_{w}=\left\{k \in[\ell(w)]: \epsilon_{k}=-\epsilon_{k+1}\right\}$, where $\epsilon$ is indexed over $\mathbb{Z} /[l(w)] \mathbb{Z}$, and an operator $\hat{L}$ on $\mathbb{C}^{\mathcal{P} W_{1}}$, setting for any $\varphi \in$ $\mathbb{C}^{\mathcal{P} W_{1}}$ and $x \in \mathcal{P} W_{1}$,

$$
\hat{L}(\varphi)(x)=\sum_{(a, b) \in \mathcal{N}_{w(S), 1_{n}}^{0, \pm}(1) \backslash \mathcal{I}_{w}} \varphi\left(\mathcal{T}_{a, b}^{ \pm}(S)\right) .
$$

Let us consider the function $\varphi_{0}$ on $\mathcal{P} W_{1}$ with $\varphi_{0}(S, \pi)=1$ if $\# \pi=1$, and 0 otherwise. As $2 \# \mathcal{I}_{w} \leq n$, the operator defined in (33) satisfies for any $t \in \mathbb{R}_{+}$,

$$
e^{t \tilde{L}_{1}}\left(\varphi_{0}\right)\left(w, 1_{n}\right) \leq e^{t\left(\frac{n}{2}+\hat{L}\right)}\left(\varphi_{0}\right)\left(w, 1_{n}\right)
$$

What is more,

$$
e^{t \hat{L}}\left(\varphi_{0}\right)\left(w, 1_{n}\right) \leq e^{t \tilde{L}_{1}}\left(\varphi_{0}\right)\left(x_{1}^{n}, 1_{n}\right) .
$$

For any $n \in \mathbb{N}^{*}$, let us denote here by $\varphi_{0} \in \mathbb{C}^{\mathcal{C} W_{1}}$, the constant function equal to 1 . Therefore, setting for any $s \geq 0, n \in \mathbb{N}, \rho_{s}(n)=e^{s \tilde{L}_{1}}\left(\varphi_{0}\right)\left(x_{1}^{n}, 1_{n}\right)$, for any $t \in \mathbb{R}_{+}^{q}$,

$$
\Lambda_{t}(w) \leq \prod_{f=1}^{q} \rho_{t_{f}}\left(\bar{n}_{w}(f)\right) .
$$

According to the Definition (33) of the operator $\tilde{L}_{1}$, the family of functions $(\rho .(n))_{n \geq 0}$ satisfies the following differential system: for any $n \in \mathbb{N}^{*}$ and $s \geq 0$,

$$
\frac{d}{d s} \rho_{s}(n)=\frac{n}{2} \sum_{p=1}^{n-1} \rho_{s}(p) \rho_{s}(n-p)
$$

and $\rho_{0}(n)=1$. Let us define a formal power series by setting for any $z$,

$$
\rho_{S}(z)=\sum_{n \geq 1} \rho_{s}(n) z^{n} .
$$

Then, for any $s \geq 0$,

$$
\frac{d}{d s} \rho_{s}(z)=z \rho_{s}(z) \frac{d}{d z} \rho_{s}(z) .
$$

According to the Lemma 13 of [6], $\left(\rho_{s}(n)\right)_{n \geq 0}$ is the sequence of moments of a Hermitian operator (therein, denoted by $\Lambda_{s / 2} \Lambda_{s / 2}^{*}$ ) acting on a separable Hilbert space and, according to Proposition 11 of the same article, with spectrum $\left[\lambda_{s / 2}^{-}, \lambda_{s / 2}^{+}\right]$, where

$$
\left[\lambda_{s}^{-}, \lambda_{s}^{+}\right]=\left[(2 s+1-2 \sqrt{s(1+s)}) e^{-\sqrt{s(s+1)}},(2 s+1+2 \sqrt{s(s+1)}) e^{\sqrt{s(s+1)}}\right] .
$$

It implies that for all $n \in \mathbb{N}^{*}, \rho_{s}(n) \leq\left(\lambda_{s / 2}^{+}\right)^{n}$ and the result then follows from (*). 


\section{Applications}

5.1. Asymptotic behavior of the free energies. We give here a proof of the Theorems 1 and 2. For any function $V \in \mathbb{C}^{W_{q}}$, let us set $\|V\|_{1}=\sum_{w \in W_{q}}|V(w)|$ and $\|V\|_{\infty}=$ $\sup _{w \in W_{q}}|V(w)|$. We define $\mathcal{F}_{1, q}=\left\{V \in \mathbb{C}^{W_{q}}:\|V\|_{1}<\infty\right\}$ and $\mathcal{F}_{0, q}$ the set of functions $V \in \mathbb{C}^{W_{q}}$, with $\#\left\{w \in W_{q}: V(w) \neq 0\right\}<\infty$. For any $N \in \mathbb{N}^{*}, U_{1}, \ldots, U_{q} \in$ $\mathrm{U}(N)$ and $V \in \mathcal{F}_{1, q}$ the following sum converges almost surely and defines a random matrix

$$
V\left(U_{1}, \ldots, U_{q}\right)=\sum_{w \in W_{q}} V(w) w\left(U_{f}, 1 \leq f \leq q\right) \in M_{N}(\mathbb{C}) .
$$

Let $\left(U_{1, t}\right)_{t \geq 0}, \ldots,\left(U_{q, t}\right)_{t \geq 0}$ be $q$ independent $\mathrm{U}(N)$ Brownian motions.

Theorem 5. For $t \in \mathbb{R}_{+}^{q}$ and $V \in \mathcal{F}_{0, q}$, there exists $r_{V}>0$ and analytic functions $\varphi_{t, V},\left(\psi_{t, V, N}\right)_{N \geq 1}$ and $\psi_{t, V}$ on $D_{r_{V}}=\left\{z \in \mathbb{C}:|z|<r_{V}\right\}$, such that

$$
e^{\psi_{t, V, N}(z)}=\mathbb{E}\left[e^{z N \operatorname{Tr}\left(V\left(U_{1, t_{1}}, \ldots, U_{q, t_{q}}\right)\right)-N^{2} \varphi_{t, V}(z)}\right] \longrightarrow e^{\psi_{t, V}(z)},
$$

as $N \rightarrow \infty$, where the convergence is uniform on compact subset of $D_{r_{V}}$.

Proof. For any function $V \in \mathcal{F}_{1, q}$, let us define $V_{t, N}=V\left(U_{1, t_{1}}, \ldots, U_{q, t_{q}}\right)$ and $I_{t, V, N}(z)=N^{-2} \log \mathbb{E}\left[e^{z N \operatorname{Tr}\left(V_{t, N}\right)}\right]$. The latter analytic function admits the following Taylor expansion on a neighborhood of 0 ,

$$
\sum_{w \in W_{q}} V(w) \varphi_{t, N}(w) z+\sum_{\substack{m \geq 2 \\ w_{1}, \ldots, w_{m} \in W_{q}}} \frac{\prod_{i=1}^{m} V\left(w_{i}\right)}{m !} \varphi_{t, N}\left(w_{1}, \ldots, w_{m}\right) z^{m} .
$$

According to Proposition 1, the summands of the two sums converge pointwise as $N \rightarrow$ $\infty$. The summand of the first sum is bounded by $|z V(w)|$, so that this sum converges absolutely towards $\sum_{w \in W_{q}} V(w) \varphi_{t}(w) z$. Each coefficient of the second power series is bounded by

$$
\sum_{\substack{w_{1}, \ldots, w_{m} \in W_{q} \\ \mathfrak{T} \in \mathcal{C}_{m}}} \frac{\prod_{i=1}^{m}\left|V\left(w_{i}\right)\right|}{m !} \mathfrak{T}\left(w_{1}, \ldots, w_{m}\right) \leq \max _{a \neq b \in \operatorname{supp}(V)}\left\langle\bar{n}_{a}, \bar{n}_{b}\right\rangle_{t}^{m-1} \frac{m^{m-2}}{m !}\|V\|_{1}^{m} .
$$

It follows that

$$
I_{t, V, N}(z)=\sum_{m \geq 1, w_{1}, \ldots, w_{m} \in W_{q}} \frac{z^{m} \prod_{i=1}^{m} V\left(w_{i}\right)}{m !} \varphi_{t, N}\left(w_{1}, \ldots, w_{m}\right)
$$

is well defined on $\mathrm{D}_{r_{V}}$ with

$$
\frac{1}{r_{V}}=\max _{a, b \in \operatorname{supp}(V)}\left\{\left\langle\bar{n}_{a}, \bar{n}_{b}\right\rangle_{t}\right\} e\|V\|_{1}
$$

and converges uniformly as $N \rightarrow \infty$, on its compact subset towards a limit that we denote by $\varphi_{t, V}(z)$. Let us set for any $V \in \mathbb{C}^{W_{q}}$,

$$
\eta_{V}=\sup _{a \in \operatorname{supp}(V)}\left\|\bar{n}_{a}\right\|_{t}^{2} \sum_{w \in W_{q}} \lambda_{t}\left(w_{i}\right)\left|V\left(w_{i}\right)\right|,
$$


where $\lambda_{t}$ is defined in (32). For any $m \geq 1$ and $V$ with $\eta_{V}<\infty$, according to Lemma 8 , the sum

$$
\psi_{N, m}(V)=\sum_{w_{1}, \ldots, w_{m} \in W_{q}} \frac{\prod_{i=1}^{m} V\left(w_{i}\right)}{m !} \psi_{t, N}\left(w_{1}, \ldots, w_{m}\right)
$$

is well defined and satisfies

$$
\left|\psi_{N, m}(V)\right| \leq 2^{m-1} \frac{m^{m}}{m !} \eta_{V}^{m}
$$

Thanks to Proposition 1 , by dominated convergence, if $\psi_{t, V, N}(z)$ denotes the power series with coefficients $\left(\psi_{N, m}(V)\right)_{m \geq 1}$, then $\psi_{t, V, N}$ is well defined on $D_{r_{V}^{\prime}}$ and converges uniformly on its compact subsets towards a function $\psi_{t, V}$, with

$$
\frac{1}{r_{V}^{\prime}}=2 e \eta_{V}
$$

To conclude, note that $r_{V}^{\prime}<r_{V}$, so that if $|z|<r_{V}^{\prime}<\infty, \varphi_{t, V}(z)$ is well defined and the analytic function

$$
e^{\psi_{t, V, N}(z)}=\mathbb{E}\left[e^{z N \operatorname{Tr}\left(V_{t, N}\right)-N^{2} \varphi_{t, V}(z)}\right]
$$

converges uniformly towards $e^{\psi_{t, V}(z)}$ on $D_{r_{V}^{\prime}}$.

For any function $V \in \mathbb{C}^{W_{q}}$ and any word $w=x_{i_{1}}^{\epsilon_{1}} \ldots x_{i_{n}}^{\epsilon_{n}} \in W_{q}$, with $\epsilon_{1}, \ldots, \epsilon_{n} \in$ $\{-1,1\}$, let us set $V^{*}(w)=\overline{V\left(x_{i_{n}}^{-\epsilon_{n}} \ldots x_{i_{1}}^{-\epsilon_{1}}\right)}$. We say that $V \in \mathbb{C}^{W_{q}}$ is symmetric if $V^{*}=V$. For any $N \in \mathbb{N}^{*}$, let $\left(U_{1, t}\right)_{t \geq 0}, \ldots,\left(U_{q, t}\right)_{t \geq 0}$ be $q$ independent $\mathrm{U}(N)$ Brownian motions. For any symmetric function $V \in \mathcal{F}_{1, q}$ and $t \in \mathbb{R}_{+}^{*}$, the random matrix

$$
V_{t, N}=V\left(U_{1, t_{1}}, \ldots, U_{q, t_{q}}\right)
$$

is Hermitian and its operator norm is bounded by $\|V\|_{1}$. In particular, it satisfies $0<\mathbb{E}\left[e^{N \operatorname{Tr}\left(V_{t, N}\right)}\right]<\infty$. Let $\mu_{t, V}$ be the probability measure on $\mathrm{U}(N)^{q}$, absolutely continuous with respect to the law of $\left(U_{1, t_{1}}, \ldots, U_{q, t_{q}}\right)$, whose Radon-Nikodym derivative is $\mathbb{E}\left[e^{N \operatorname{Tr}\left(V_{t, N}\right)}\right]^{-1} e^{N \operatorname{Tr}\left(V\left(U_{1}, \ldots, U_{q}\right)\right)}$. We shall denote by $\left(U_{1, t_{1}}^{V}, \ldots, U_{q, t_{q}}^{V}\right)$ a random variable distributed as $\mu_{t, V}$ on $\mathrm{U}(N)^{q}$.

For any $V, W \in \mathbb{C}^{W_{q}}$, let us define for any $t \in \mathbb{R}_{+}^{q}$,

$$
\mathcal{J}_{t, V}(W)=\sum_{m \geq 1, a, w_{1}, \ldots, w_{m} \in W_{q}, \mathfrak{T} \in \mathcal{C}_{m}}|W(a)| \frac{\prod_{i=1}^{m}\left|V\left(w_{i}\right)\right|}{m !} \mathfrak{T}_{t}\left(a, w_{1}, \ldots, w_{m}\right)
$$

and

$$
\begin{aligned}
& \mathcal{J}_{t, 2, V}(W)=\sum_{a, b \in W_{q}}\left\langle\bar{n}_{a}, \bar{n}_{b}\right\rangle_{t}|W(a)||W(b)| \\
& +\sum_{m \geq 1, a, b, w_{1}, \ldots, w_{m} \in W_{q}, \mathfrak{T} \in \mathcal{C}_{m}}|W(a)||W(b)| \frac{\prod_{i=1}^{m}\left|V\left(w_{i}\right)\right|}{m !} \mathfrak{T}_{t}\left(a, b, w_{1}, \ldots, w_{m}\right) .
\end{aligned}
$$

For a fixed $V \in \mathbb{C}^{W_{q}}, t \in \mathbb{R}_{+}^{q}$, we consider $\mathcal{F}_{t, V}=\left\{W \in \mathcal{F}_{1, q}: \mathcal{J}_{t, V}, \mathcal{J}_{t, 2, V}<\infty\right\}$. 
Theorem 6. For any $V \in \mathcal{F}_{1, q}$, the random variables $\frac{1}{N} \operatorname{Tr}\left(W\left(U_{1, t_{1}}^{V}, \ldots, U_{q, t_{q}}^{V}\right)\right)$, with $W \in \mathcal{F}_{t, V}$ converge jointly in probability towards constants $\Phi_{t, V}(W), W \in \mathcal{F}_{t, V}$.

Remark 4. For any Cayley tree $\mathfrak{T} \in \mathcal{C}_{m}$, let us write $\left(d_{\mathfrak{T}}(i)\right)_{1 \leq i \leq m}$ for the degree distribution of $\mathfrak{T}$. For $m \geq 2$, Cauchy-Schwarz inequality yields that for $w_{1}, \ldots, w_{m} \in W_{q}$,

$$
\frac{\prod_{i=1}^{m}\left|V\left(w_{i}\right)\right|}{m !} \mathfrak{T}_{t}\left(w_{1}, \ldots, w_{m}\right) \leq \sum_{\mathfrak{T} \in \mathcal{C}_{m}} \frac{1}{m !} \prod_{i=1}^{m}\left|V\left(w_{i}\right)\right|\left\|\bar{n}_{w_{i}}\right\|_{t}^{d_{\mathfrak{T}}(i)}
$$

For any $V \in \mathbb{C}^{W_{q}}$, according to formula (1) of [4],

$$
\sum_{w \in W_{q}^{m}} \prod_{i=1}^{m}\left|V\left(w_{i}\right)\right| \mathfrak{T}_{t}\left(w_{1}, \ldots, w_{m}\right) \leq \sum_{w \in W_{q}^{m}} \prod_{i=1}^{m}\left\|\bar{n}_{w_{i}}\right\|_{t}\left|V\left(w_{i}\right)\right|\left(\sum_{i=1}^{m}\left\|\bar{n}_{w_{i}}\right\|_{t}\right)^{m-2} .
$$

In particular, $\mathcal{J}_{t, V}(W), \mathcal{J}_{t, 2, V}(W)<\infty$, as soon as $V, W \in \mathcal{F}_{0, q}$, with

$$
\|V\|_{1} . \sup _{\substack{a \in W_{q} \\ W(a) \text { or } V(a) \neq 0}}\left\|\bar{n}_{a}\right\|_{t}^{2}<\frac{1}{e} .
$$

Proof. Let us consider $V \in \mathcal{F}_{1, q}$ and $W \in \mathcal{F}_{t, V}$. According to (3), (4) and Proposition 2, the mean and variance of the random variable $\frac{1}{N} \operatorname{Tr}\left(W\left(U_{1, t_{1}}^{V}, \ldots, U_{q, t_{q}}^{V}\right)\right.$ are respectively equal to the following absolutely convergent sums,

$$
\sum_{\substack{m \geq 0, w, w_{1}, \cdots, w_{m} \in W_{q}}} \frac{1}{m !} W(w) \prod_{k=1}^{m} V\left(w_{k}\right) \varphi_{t, N}\left(w, w_{1}, \ldots, w_{m}\right)
$$

and

$$
\sum_{\substack{m \geq 0, a, b, w_{1}, \cdots, w_{m} \in W_{q}}} \frac{1}{m !} W(a) W(b) \prod_{k=1}^{m} V\left(w_{k}\right) \varphi_{t, N}\left(a, b, w_{1}, \ldots, w_{m}\right) .
$$

According to Proposition 2, dominated convergence implies that these two sequences have a limit as $N \rightarrow \infty$.

\subsection{Central limit theorem. As a consequence of Proposition 1, we get the}

Proposition 3. For any $t \in \mathbb{R}_{+}^{q}$, using the notation (13), the family $\left(\operatorname{Tr}\left(w_{t}^{N}\right)-\right.$ $\left.N \varphi_{t}(w)\right)_{w \in W_{q}}$, converges towards the centered Gaussian field $\left(\phi_{w}\right)_{w \in W_{q}}$, such that for any $a, b \in W_{q}, \operatorname{cov}\left(\phi_{w}, \phi_{w^{\prime}}\right)=\varphi_{t}\left(w, w^{\prime}\right)$.

Proof. For any word $w \in W_{q}, \mathbb{E}\left[N^{-1} \operatorname{Tr}\left(w_{t}^{N}\right)\right]=\varphi_{t}(w)+O\left(N^{-2}\right)$, whereas for any $m \geq 2$ and $w \in W_{q}^{m}, C_{m}\left(\operatorname{Tr}\left(w_{1, t}^{N}\right), \ldots, \operatorname{Tr}\left(w_{m, t}^{N}\right)\right)=N^{2-m} \varphi_{t, N}\left(w_{1}, \ldots, w_{m}\right)$ converges, as $N \rightarrow \infty$, towards $\varphi_{t}\left(w_{1}, w_{2}\right)$, if $m=2$, and 0 , if $m \geq 3$. 
Let us remark that in the proof of Proposition 2, we obtained in formula (31) an expression of the function $\varphi_{t, N}$ for any $N \in \mathbb{N}^{*}$, in terms of its restriction to single words. Specialized to partitioned words with two blocks, this gives an expression of the covariance of the above field. Let us define a family of $3 q$ independent $\mathrm{U}(N)$ Brownian motions $\left(U_{1, t}\right)_{t \geq 0},\left(V_{1, t}^{1}\right)_{t \geq 0},\left(V_{1, t}^{2}\right)_{t \geq 0}, \ldots,\left(U_{q, t}\right)_{t \geq 0},\left(V_{q, t}^{1}\right)_{t \geq 0},\left(V_{q, t}^{2}\right)_{t \geq 0}$. For any words $w_{1}, w_{2} \in W_{q}$ and $(a, b) \in \mathcal{N}_{w_{1} w_{2}}(f) \backslash \mathcal{S}_{\left(w_{1}, w_{2}\right), 0_{2}}$, let $\chi:\left[\ell\left(w_{1} w_{2}\right)\right] \rightarrow$ $\{1,2\}$ be the function such that the $i^{\text {th }}$ letter of the word $\mathcal{T}_{a, b}\left(\left(w_{1}, w_{2}\right)\right)$ belongs to $w_{\chi(i)}$. If $\mathcal{T}_{a, b}\left(\left(w_{1}, w_{2}\right)\right)=x_{i_{1}}^{\epsilon_{1}} \ldots x_{i_{n}}^{\epsilon_{n}}$, let us set for any $r, s \in \mathbb{R}_{+}^{q}$,

$$
w_{a, b}(r, s)=\left(U_{i_{1}, r_{i_{1}}} V_{i_{1}, s_{i_{1}}}^{\chi(1)}\right)^{\epsilon_{1}} \ldots\left(U_{i_{n}, r_{i_{n}}} V_{i_{n}, s_{i_{n}}}^{\chi(n)}\right)^{\epsilon_{n}} .
$$

According to formula (31),

$$
\operatorname{cov}\left(\operatorname{Tr}\left(w_{1, t, N}\right), \operatorname{Tr}\left(w_{2, t, N}\right)\right)=\sum_{\substack{1 \leq f \leq q \\ \epsilon \in\{-1,1\}}} \epsilon t_{f} \int_{0}^{1} \sum \frac{1}{N} \mathbb{E}\left[\operatorname{Tr}\left(w_{a, b}(s t,(1-s) t)\right)\right] d s,
$$

where the second sum is over pairs $(a, b) \in \mathcal{N}_{w_{1} w_{2}}^{\epsilon}(f) \backslash \mathcal{S}_{\left(w, w^{\prime}\right), 0_{2}}$.

\section{Planar Yang-Mills Measure}

We shall see in this section how the results of the previous one apply in the framework of the planar Yang-Mills measure. We first recall a construction and some properties of the planar Yang-Mills measure following [35,37] and from Sect. 6.7 on, explain our results. In the next section, we will then give analogues of Schwinger-Dyson's equations.

6.1. Paths of finite length. Let us call parametrized path any Lipschitz function from $[0,1]$ to $\mathbb{R}^{2}$, that are either constant or with speed bounded by below. We denote by $\mathrm{P}\left(\mathbb{R}^{2}\right)$ the set of parametrized paths up to bi-Lipschitz increasing reparametrization and call its elements paths. For any path $\gamma \in \mathrm{P}\left(\mathbb{R}^{2}\right)$ with parametrization $c:[0,1] \rightarrow \mathbb{R}^{2}$, let us denote its endpoints $c(0)$ and $c(1)$ by $\underline{\gamma}$ and $\bar{\gamma}$, and by $\gamma^{-1}$ the inverse path parametrized by $t \in[0,1] \mapsto c(1-t)$. For any $x \in \mathbb{R}^{2}$, we denote by $\mathrm{L}_{x}\left(\mathbb{R}^{2}\right)$ the set of paths $\gamma \in \mathrm{P}\left(\mathbb{R}^{2}\right)$ such that $\gamma=x=\bar{\gamma}$ and call elements of $\mathrm{L}_{x}\left(\mathbb{R}^{2}\right)$ loops based at $x$. We set $\mathrm{L}\left(\mathbb{R}^{2}\right)=\cup_{x \in \mathbb{R}^{2}} L_{x}\left(\mathbb{R}^{2}\right)$. For any loop $l$ based at some point $x \in \mathbb{R}^{2}$ and parametrized by the Lipschitz-continuous map $\tilde{l}:[0,1] \rightarrow \mathbb{R}^{2}$, we call non-based loop the induced map $\mathbb{U} \rightarrow \mathbb{R}^{2}$, up to bi-Lipschitz, order preserving, one-to-one mappings of $\mathbb{U}$. If $a$ and $b$ are two paths such that $\bar{a}=\underline{b}$, we denote by $a b$ the path of $\mathrm{P}\left(\mathbb{R}^{2}\right)$ obtained by concatenation.

6.2. Embedded graphs. We call here embedded graph in the plane the data of a triple of finite sets $\mathbb{G}=(\mathbb{V}, \mathbb{E}, \mathbb{F})$, where faces $\mathbb{F}$ are domains of the plane with disjoint interior, simply connected in the Riemann sphere $\hat{\mathbb{C}}$ and which boundary is the image of a non-based loops, edges $\mathbb{E}$ are paths of $\mathrm{P}\left(\mathbb{R}^{2}\right)$ stable by the inversion map, such that the union of their image is the union of boundaries of elements of $\mathbb{F}$, vertices $\mathbb{V}$ are the endpoints of $\mathbb{E}$ and the graph induced by $\mathbb{E}$ on $\mathbb{V}$ is connected. With this convention, any edge $e \in \mathbb{E}$ is either a simple loop or an injective path of finite length. As any edge is bounded, $\mathbb{G}$ has a unique unbounded face that we denote by $F_{\infty, \mathbb{G}}$ (or simply $F_{\infty}$ ) and set $\mathbb{F}_{b}=\mathbb{F} \backslash F_{\infty}$. We write $|F|$ the area of any element of $F \in \mathbb{F}_{b}$ and by $\partial F$ the 
non-based loop, whose image is the boundary of $F$ with counterclockwise orientation. We shall write $\mathrm{P}(\mathbb{G})$ for the set of paths that are concatenation of elements of $\mathbb{E}$ and $\mathrm{L}(\mathbb{G})$ (and respectively for any $v \in \mathbb{V}, \mathrm{L}_{v}(\mathbb{G})$ ) for the set of loops (respectively loops based at $v$ ) in $\mathrm{P}(\mathbb{G})$. The dual graph of $\mathbb{G}$ is the combinatorial graph $\hat{\mathbb{G}}=(\mathbb{F}, \hat{\mathbb{E}})$ with vertices $\mathbb{F}$, where two faces are neighbors if their closures intersect. For any path $\gamma \in \hat{\mathbb{G}}$, we denote its number of edges by $|\gamma|$.

6.3. A free group: reduced loops of an embedded graph. Let us fix an embedded graph $\mathbb{G}$. For any pair of paths $\gamma_{1}$ and $\gamma_{2}$ of $\mathrm{P}(\mathbb{G})$, let us write $\gamma_{1} \sim \gamma_{2}$ and say that $\gamma_{1}$ and $\gamma_{2}$ are equivalent, if one can get $\gamma_{1}$ from $\gamma_{2}$, or vice-versa, by adding or erasing paths of the form $e . e^{-1}$, with $e \in \mathbb{E}$. For any path $\gamma$, there is a unique element of minimal length in its equivalence class, that we call the reduction of $\gamma$. The set of reduced paths endowed with the operation of concatenation and reduction forms a groupoid that we denote by $\operatorname{RP}(\mathbb{G})$. For any $v \in \mathbb{V}$, we denote by $\mathrm{RL}_{v}(\mathbb{G})$ the set of reduced paths that are loops based at $v$. Endowed with the above multiplication, $\operatorname{RL}_{v}(\mathbb{G})$ is a free group of rank $\# \mathbb{F}_{b}$ (we shall highlight specific free basis in Sect. 6.6).

6.3.1. Multiplicative functions. For any $N \in \mathbb{N}^{*}$ and any subset $\mathfrak{P}$ of $\mathrm{P}\left(\mathbb{R}^{2}\right)$, stable by concatenation, we call a function $h: \mathfrak{P} \rightarrow \mathrm{U}(N)$, multiplicative if for any paths $a, b \in \mathfrak{P}$, with $\bar{a}=\underline{b}$,

$$
h(a b)=h(a) h(b) .
$$

We denote the space of multiplicative functions by $\mathcal{M}_{N}(\mathfrak{P})$ and by $\mathcal{C}_{\mathfrak{P}}$ (or simply $\mathcal{C}$, when $\mathfrak{P}=\mathcal{P}\left(\mathbb{R}^{2}\right)$ ) the smallest $\sigma$-fields such that for any $\gamma \in \mathfrak{P}, h \in \mathcal{M}_{N}(\mathfrak{P}) \longmapsto$ $h(\gamma) \in U(N)$ is measurable, where $U(N)$ is endowed with its Borel $\sigma$-fields. For any embedded graph $\mathbb{G}, v \in \mathbb{V}$ and any choice of basis $\Lambda$ of $\mathrm{RL}_{v}(\mathbb{G})$, there is a bijection

$$
\begin{aligned}
\Theta_{\Lambda}: \mathcal{M}_{N}\left(\mathrm{~L}_{v}(\mathbb{G})\right) & \rightarrow \mathrm{U}(N)^{\# \mathbb{F}_{b}} \\
h & \mapsto(h(\lambda))_{\lambda \in \Lambda} .
\end{aligned}
$$

6.3.2. Lassos basis and discrete Yang-Mills measure. For any loop $v \in \mathbb{V}$ and $l \in$ $\mathrm{L}_{v}(\mathbb{G})$, we say that $l$ is a lasso based at $v$, if $l=a \partial_{\bar{a}} F a^{-1}$ where $a \in \mathrm{P}\left(\mathbb{R}^{2}\right)$, with $a=v$, $\bar{a}$ is a vertex in the image of $\partial F$ and $\partial_{\bar{a}} F$ is the rooting of $\partial F$ at $\bar{a}$. We shall see in the next section that there exists basis of $\mathrm{RL}_{v}(\mathbb{G})$ formed with lassos. Let $\Lambda=\left(\lambda_{F}\right)_{F \in \mathbb{F}_{b}}$ be a free basis of $\mathrm{RL}_{v}(\mathbb{G})$, composed with lassos, and let $Y M_{\mathbb{G}}^{\Lambda}$ be the law of $\Theta_{\Lambda}^{-1}\left(\left(U_{F,|F|}\right)_{F \in \mathbb{F}_{b}}\right)$ on $\left(\mathcal{M}_{N}\left(\mathrm{~L}_{v}(\mathbb{G})\right), \mathcal{C}_{\mathbb{G}}\right)$, where $\left(\left(U_{F, t}\right)_{t \geq 0}\right)_{F \in \mathbb{F}_{b}}$ is a family of independent Brownian motions on $\mathrm{U}(N)$.

Lemma 10 [35]. (i) For any lassos free basis $\Lambda, \Lambda^{\prime}$ of $\mathrm{RL}_{v}(\mathbb{G}), Y M_{\mathbb{G}}^{\Lambda}=Y M_{\mathbb{G}}^{\Lambda^{\prime}}$. We denote this law by $Y M_{\mathbb{G}}$.

(ii) If $\mathbb{G}^{\prime}$ is embedded graph with $\mathrm{P}\left(\mathbb{G}^{\prime}\right) \subset \mathrm{P}(\mathbb{G})$ and $\mathcal{R}_{\mathbb{G}^{\prime}}^{\mathbb{G}}: \mathcal{M}_{N}\left(\mathrm{~L}_{v}(\mathbb{G})\right) \rightarrow$ $\mathcal{M}_{N}\left(\mathrm{~L}_{v}\left(\mathbb{G}^{\prime}\right)\right)$ denotes the restriction map, then

$$
\mathcal{R}_{\mathbb{G}^{\prime} *}^{\mathbb{G}} Y M_{\mathbb{G}}=Y M_{\mathbb{G}^{\prime}}
$$

We denote by $\left(H_{l}\right)_{l \in \mathrm{L}_{v}(\mathbb{G})}$ the canonical process on $\mathcal{M}_{N}\left(\mathrm{~L}_{v}(\mathbb{G})\right)$ with law $\mathrm{YM}_{\mathbb{G}}$. The first point follows from the invariance of the law of the $\mathrm{U}(N)$-Brownian motion by adjunction and the following result. 
Theorem 7 [30]. Let $X=\left(x_{1}, \ldots, x_{n}\right)$ and $Y=\left(y_{1}, \ldots, y_{n}\right)$ be two free basis for the free group $F_{n}$, such that $x_{i}$ is conjugated to $y_{i}$ for all $i \in[n]$. Then $X$ can be obtained from $Y$ by a sequence of transformations of the kind $\left(u_{1}, \ldots, u_{n}\right) \mapsto\left(u_{1}^{\prime}, \ldots, u_{n}^{\prime}\right)$ where, for some $i, j, u_{i}^{\prime}=u_{j} u_{i} u_{j}^{-1}$ or $u_{j}^{-1} u_{i} u_{j}$ and $u_{k}^{\prime}=u_{k}$ for $k \neq i$.

The second point of Lemma 10 requires a proof (see [37, Prop. 4.3.4]) that we won't reproduce here; an argument goes as follows. Let $\mathbb{G}^{\prime}=\left(\mathbb{V}^{\prime}, \mathbb{E}^{\prime}, \mathbb{F}_{b}^{\prime}\right)$ be an embedded graph with $v \in \mathbb{V}^{\prime}$ and $\mathrm{P}\left(\mathbb{G}^{\prime}\right) \subset \mathrm{P}(\mathbb{G})$. Assume that there exists $F \in \mathbb{F}_{b}^{\prime}$ and $F_{1}, F_{2} \in \mathbb{F}_{b}$ with $\bar{F}=\overline{F_{1} \cup F_{2}}$. If $\lambda$ and $\lambda_{1}^{\prime}, \lambda_{2}^{\prime}$ are lassos respectively in $\mathrm{L}_{v}(\mathbb{G})$ and $\mathrm{L}_{v}\left(\mathbb{G}^{\prime}\right)$, with faces $F, F_{1}, F_{2}$ such that $\lambda=\lambda_{1} \lambda_{2}$, then under $Y M_{\mathbb{G}}, H_{\lambda}$ has the same law as $U_{1,\left|F_{1}\right|} U_{2,\left|F_{2}\right|}$, where $\left(U_{1, t}\right)_{t \geq 0}$ and $\left(U_{2, t}\right)_{t \geq 0}$ are two independent $\mathrm{U}(N)$-Brownian motions. Hence, it has the same law as $U_{1,|F|}$, that is the law of $H_{\lambda}$ under $Y M_{\mathbb{G}^{\prime}}$.

6.4. Yang-Mills measure. Let $d_{1}$ and $d_{l}$ be the two distances on $\mathrm{P}\left(\mathbb{R}^{2}\right)$ defined in the following way: for any pair of paths $\gamma_{1}, \gamma_{2} \in \mathrm{P}\left(\mathbb{R}^{2}\right)$, parametrized by $c_{1}, c_{2}:[0,1] \rightarrow$ $\mathbb{R}_{2}$, with $\left|c_{1}^{\prime}\right|=\left|c_{2}^{\prime}\right|=1$,

$$
d_{1}\left(\gamma_{1}, \gamma_{2}\right)=\left|\underline{\gamma_{1}}-\underline{\gamma_{2}}\right|+\int_{0}^{1}\left|c_{1}^{\prime}(t)-c_{2}^{\prime}(t)\right| d t
$$

and

$$
d_{\ell}\left(\gamma_{1}, \gamma_{2}\right)=\inf _{\phi, \psi} \sup _{r, s \in[0,1]}\left\{\left|c_{1} \circ \phi(r)-c_{2} \circ \psi(s)\right|\right\}+\left|\ell\left(c_{1}\right)-\ell\left(c_{2}\right)\right|
$$

where we have denoted by $\ell(c)$ the length of a path $\gamma \in \mathrm{P}\left(\mathbb{R}^{2}\right)$ and the infimum is taken over all increasing bijections of [0,1]. It has been proved in [37] that $d_{1}$ and $d_{\ell}$ induce the same topology on $\mathrm{P}\left(\mathbb{R}^{2}\right)$, though $\left(\mathrm{P}\left(\mathbb{R}^{2}\right), d_{1}\right)$ is complete and $\left(\mathrm{P}\left(\mathbb{R}^{2}\right), d_{\ell}\right)$ is not. In the following, we shall only use this topology and say that a sequence of paths $\left(l_{n}\right)_{n \geq 0}$ converges to $l$ if $d_{\ell}\left(\gamma_{n}, \gamma\right) \rightarrow 0$ and $\gamma_{n}=\gamma, \overline{\gamma_{n}}=\bar{\gamma}$, for every $n \in \mathbb{N}^{*}$. For any embedded graph $\mathbb{G}$, with $v \in \mathbb{V}$, let us denote by $\mathcal{R}_{\mathbb{G}}^{v}: \mathcal{M}_{N}\left(\mathrm{P}\left(\mathbb{R}^{2}\right)\right) \rightarrow \mathcal{M}_{N}\left(\mathrm{~L}_{v}(\mathbb{G})\right)$ the restriction mapping. This application is measurable with respect to the $\sigma$-fields $\mathcal{C}$ and $\mathcal{C}_{\mathbb{G}}$. It is shown in [37] that the family of measures $Y M_{\mathbb{G}}$, with $\mathbb{G}$ ranging over embedded graphs, can be extended in the following way.

Theorem 8. There exists a probability measure $\mathrm{YM}_{N}$ on $\left(\mathcal{M}_{N}\left(\mathrm{P}\left(\mathbb{R}^{2}\right)\right), \mathcal{C}\right)$ such that, for any embedded graph $\mathbb{G}, v \in \mathbb{R}^{2}$,

$$
\mathcal{R}_{\mathbb{G} *}^{v} \mathrm{YM}_{N}=Y M_{\mathbb{G}}
$$

Let $\left(H_{\gamma}\right)_{\gamma \in \mathrm{P}\left(\mathbb{R}^{2}\right)}$ be a random multiplicative function with law $\mathrm{YM}_{N}$. If $\left(\gamma_{n}\right)_{n \geq 0}$ is a sequence of paths in $\mathrm{P}\left(\mathbb{R}^{2}\right)$ that converges to $\gamma$, then, under $\mathrm{YM}, H_{\gamma_{n}}$ converges in probability towards $H_{\gamma}$. If $h$ is an area preserving diffeomorphism of $\mathbb{R}^{2}$, then the process $\left(H_{h(\gamma)}\right)_{\gamma \in \mathrm{P}\left(\mathbb{R}^{2}\right)}$ and $\left(H_{\gamma}\right)_{\gamma \in \mathrm{P}\left(\mathbb{R}^{2}\right)}$ have the same law.

For any $l \in \mathrm{L}\left(\mathbb{R}^{2}\right)$ and $N \in \mathbb{N}^{*}$, the random variable $\frac{1}{N} \operatorname{Tr}\left(H_{l}\right)$ is called a Wilson loop. The above approach to define the Yang-Mills measure on embedded graphs by defining a random morphism of the free group has been considered by Franck Gabriel to give a different construction of the Yang-Mills measure and to solve problems of characterization of Markovian holonomy fields in [23]. Therein, one of the key feature 
is the study of group valued sequences satisfying properties of invariances by an action of the braid group (analogues to the operations introduced in Theorem 7). In [10,23], it is also the starting point of an alternative construction of the Yang-Mills measure and of master fields in the plane.

6.5. U(1)-Yang-Mills measure. Let us consider the commutative case, $N=1$. Let $\mathbb{G}$ be an embedded graph in the plane, $v$ a vertex of $\mathbb{G}$. For any loop $l \in \operatorname{RL}_{v}(\mathbb{G})$, its winding number function defines a compactly supported function $n_{l} \in \mathrm{L}^{2}\left(\mathbb{R}^{2}\right)$. Let us fix a family of lassos $\left(\lambda_{F}\right)_{F \in \mathbb{F}_{b}}$ of $\mathbb{G}$. Under $\mathrm{YM}_{1}$ measure, $\left(H_{\lambda_{F}}\right)_{F \in \mathbb{F}_{b}}$ has the same law as $\# \mathbb{F}_{b}$ independent marginals of $\mathrm{U}(1)$-Brownian motion $\left(U_{F,|F|}\right)_{F \in \mathbb{F}_{b}}$. Let $W$ be a white noise on the plane, with intensity given by the Lebesgue measure. The random family $\left(H_{l}\right)_{l \in \mathrm{RL}_{v}(\mathbb{G})}$ is equal to $\left(\prod_{F \in \mathbb{F}_{b}} H_{\lambda_{F}}^{n_{F}(l)}\right)_{l \in \mathrm{RL}_{v}(\mathbb{G})}$ and has the same

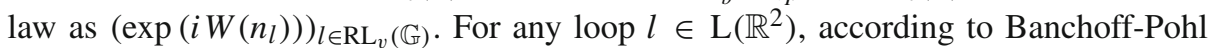
inequality (see Lemma 15), its winding number function defines an element $n_{l} \in \mathrm{L}\left(\mathbb{R}^{2}\right)$. Moreover, according to Theorem 3.3.1. of [37], the map $l \in \mathrm{L}_{0}\left(\mathbb{R}^{2}\right) \rightarrow \mathrm{L}^{2}\left(\mathbb{R}^{2}\right)$ is continuous, so that, if $\left(l_{n}^{1}\right)_{n \geq 0}, \ldots,\left(l_{n}^{m}\right)_{n \geq 0}$ are sequences of $\mathrm{L}_{0}\left(\mathbb{R}^{2}\right)$ that converge for the $d_{1}$ topology to a family of loops $\left(l_{k}\right)_{1 \leq l \leq m} \in \mathrm{L}_{0}\left(\mathbb{R}^{2}\right)^{m}$, the sequences of random variables $\exp \left(i W\left(n_{l_{n}^{1}}\right)\right), \ldots, \exp \left(i W\left(n_{l_{n}^{m}}\right)\right)$ converge jointly to $\left(\exp \left(i W\left(n_{l^{k}}\right)\right)\right)_{1 \leq k \leq m}$ in distribution. Hence, the process $\left(H_{l}\right)_{l \in \mathrm{L}\left(\mathbb{R}^{2}\right)}$ introduced in Theorem 8 has the same law as $\left(\exp \left(i W\left(n_{l}\right)\right)\right)_{l \in \mathrm{L}\left(\mathbb{R}^{2}\right)}$. Moreover, the same argument and Lemma 2 yield the following lemma.

Lemma 11. For any integer $N \in \mathbb{N}^{*}$, under $\mathrm{YM}_{N}$, the law of $\left(\operatorname{det}\left(H_{l}\right)\right)_{l \in \mathrm{L}_{0}\left(\mathbb{R}^{2}\right)}$ and $\left(\exp \left(i W\left(n_{l}\right)\right)\right)_{l \in \mathrm{L}_{0}\left(\mathbb{R}^{2}\right)}$ is $Y M_{1}$.

6.6. Two free basis of the group of reduced loops. We shall present two families of free basis of $\mathrm{RL}_{v}(\mathbb{G})$. Let $\mathbb{E}^{+}$be an orientation of $\mathbb{G}$, that is a subset of $\mathbb{E}$ such that for any $e \in \mathbb{E}, e$ or $e^{-1} \in \mathbb{E}^{+}$. Let us also fix a spanning tree $T$ of the graph $\mathbb{G}$ and set $T^{+}$ the collection of positively oriented edges of $T$. We denote by $e: \mathbb{F}_{b} \rightarrow \mathbb{E}^{+} \backslash T^{+}$the unique bijection such that for any face $F \in \mathbb{F}_{b}, e(F)$ is bounding the face $F$. For any $e \in \mathbb{E}$, bounding a face $F$, we denote by $\partial_{e} F$ the loop starting with $e$ and bounding $F$. For any $x, y \in \mathbb{V}$, we denote by $[x, y]_{T}$ the unique path in $T$ going from $x$ to $y$. Let us now define two families of loops by setting for any edge $e \in \mathbb{E}$,

$$
\beta_{e}=[v, \underline{e}]_{T} e[\bar{e}, v]_{T}
$$

and for any face $F \in \mathbb{F}_{b}$,

$$
\lambda_{F}=[v, \underline{e(F)}]_{T} \partial_{e(F)} F[\underline{e(F)}, v]_{T} .
$$

It is easy to see that $\mathrm{RL}_{v}(\mathbb{G})$ is a free group of rank $\# \mathbb{F}_{b}$ with free basis $\left(\beta_{e}\right)_{e \in \mathbb{E}^{+} \backslash T^{+}}$. For any loop $l \in \mathrm{L}(\mathbb{G})$,

$$
l \sim \beta_{e_{1}} \beta_{e_{2}} \cdots \beta_{e_{n}},
$$

where $e_{1}, \ldots, e_{n}$ are the edges in $\mathbb{E} \backslash T$, used by the loop $l$ in this order. In [35], it is proved that the second family of loops is another free basis of $\operatorname{RL}_{v}(\mathbb{G})$.

Lemma 12 [35]. The family $(\lambda)_{F \in \mathbb{F}_{b}}$ is a free basis of $\mathrm{RL}_{v_{0}}(\mathbb{G})$. 


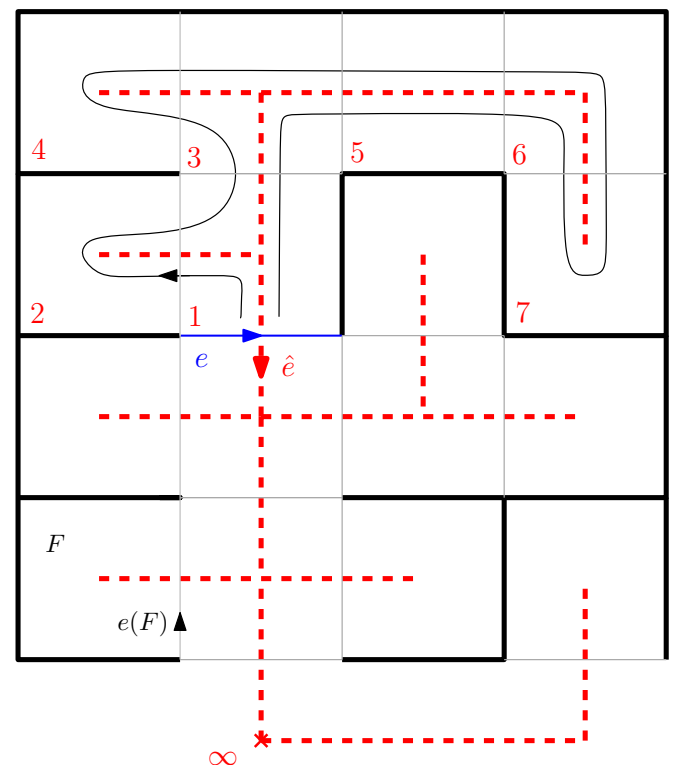

Fig. 3. We represent with black lines a spanning tree of a square grid together with its dual with dashed red lines. We also display an edge $e$ of $\mathbb{E}^{+} \backslash T$ in blue together with the order $\preccurlyeq e$ on $\hat{T}_{F_{L}(e)}$, by numbering its elements and drawing in black the clockwise contour process around $\hat{T}_{F_{L}(e)}$. Here, elements of $C_{F_{L}(e)}$ are labeled 2 and 3 (color figure online)

For any edge $e \in \mathbb{E}$, we denote by $F_{L}(e)$ and $F_{R}(e)$ the edges on the left and on the right of $e$ and denote by $\hat{e}$ the edge $\left(F_{L}(e), F_{R}(e)\right) \in \hat{\mathbb{E}}$ in the dual graph. Let $\hat{T}=\mathbb{E} \backslash T$ be the dual spanning tree of $T$, considered as rooted at the infinite face $F_{\infty}$. We fix an orientation $\mathbb{E}^{+}$of $\mathbb{G}$, such that for any edge $e \in \mathbb{E}^{+} \backslash T$, the distance in $\hat{T}$ to the root $F_{\infty}$ decreases along $\hat{e}$. Note that with this orientation, for any bounded face $F$, $F_{L}(e(F))=F$. For any face $F$, we denote by $\hat{T}_{F}$ the subtree of $\hat{T}$, with root $F$ and vertices the set of descendants of $F$ in $\hat{T}$. We denote by $C_{F}$ the set of children of $F$. For any edge $e \in \mathbb{E}^{+} \backslash T^{+}, \hat{T}_{F_{L}(e)}$ is endowed with the order $\preccurlyeq e$ induced by the time of the first visit by the clockwise contour process boarding the dual tree $\hat{T}$, starting along the left of $\hat{e}^{-1}$, as is displayed with an example in Fig. 3. Then, for any edge $e \in \mathbb{E}^{+} \backslash T$,

$$
\lambda_{F_{L}(e)}=\beta_{e}\left(\prod_{F \in C_{F_{L}(e)}} \beta_{e(F)}\right)^{-1}
$$

and

$$
\beta_{e}=\prod_{F \in \hat{T}_{F_{L}(e)}} \lambda_{F},
$$

where $\vec{\prod}$ denotes the product of terms increasing for $\preccurlyeq e$, from the left to the right. For any loop $l \in \mathrm{L}_{v}(\mathbb{G})$, we denote by $w_{l}^{T}$ the word with letters $\left(\lambda_{F}\right)_{F \in \mathbb{F}_{b}}$ and their inverse, such that $l \sim w$, given by the decomposition (35) and the inversion formula (36). Using notation (11), for any face $F \in \mathbb{F}_{b}$ and any complex number $z \in F$, the winding number satisfies

$$
n_{l}(z)=n_{w_{l}}(F) \text {. }
$$


6.6.1. Complexity of lassos decompositions. We can now give an estimate on the complexity of the above decomposition of a loop in $\mathbb{G}$ in a word of lassos associated to a spanning tree $T$. We display here results of [35] in a slightly different form, adapted to our purpose. Let us fix an embedded graph $\mathbb{G}=(\mathbb{V}, \mathbb{E}, \mathbb{F}), v \in \mathbb{V}$ and $t=(|F|)_{F \in \mathbb{F}_{b}}$. For any subset $E \subset \mathbb{E}$ and any loop $l \in \mathrm{L}(\mathbb{G})$, denote by $\mathcal{L}_{E}(l)$ the number of times that $l$ uses the edges of $E$ or $E^{-1}$. The two following lemmas are elementary.

Lemma 13. Let $l \in \mathrm{L}(\mathbb{G})$ be a loop of $\mathbb{G}$. Then, for any face $F \in \mathbb{F}_{b}$,

$$
\bar{n}_{w_{l}^{T}}(F)=\mathcal{L}_{\left[F, F_{\infty}\right]_{\hat{T}}}(l) .
$$

Lemma 14. There exists a spanning tree $T$ of $\mathbb{G}$, such that for any face $F \in \mathbb{F}_{b}$,

$$
d_{\hat{T}}\left(F, F_{\infty}\right)=d_{\hat{\mathbb{G}}}\left(F, F_{\infty}\right) .
$$

For any loop $l \in \mathrm{L}_{v}(\mathbb{G})$ and $T$ a spanning tree of $\mathbb{G}$, we want to control the maximal Amperean area $A_{t}\left(w_{l}^{T}\right)=\sum_{F \in \mathbb{F}}|F| \bar{n}_{w_{l}^{T}}(F)^{2}$, with the length of the loop $\ell(l)$. The Amperean area of $l$ is the integral

$$
A(l)=\int_{\mathbb{R}^{2}} n_{l}(x)^{2} d x .
$$

Lemma 15 (Banchoff-Pohl inequality [2]). For any loop of finite length $l \in \mathrm{L}\left(\mathbb{R}^{2}\right)$,

$$
A(l) \leq \pi \ell(l)^{2}
$$

Note that if $\bar{n}_{w_{l}^{T}}= \pm n_{l} \in \mathbb{Z}^{\mathbb{F}}$, that is, if $l$ winds only to the left or only to the right, then the Banchoff-Pohl inequality gives the expected bound. To treat more general loops, we need the following lemma.

Lemma 16 [35, 3. Lemma 5.9]. There exists a loop $\bar{l} \in \mathrm{L}(\mathbb{G})$, which does not use any edge twice, such that for any face $F \in \mathbb{F}$ and $z \in F$,

$$
n_{\bar{l}}(z)=d\left(F, F_{\infty}\right)
$$

Lemma 17. Let $l \in \mathrm{L}(\mathbb{G})$ be a loop that uses each edge at most once. If $T$ is a spanning tree chosen as in Lemma 14 and $\mathbb{E}^{+}$is any orientation of $\mathbb{G}$, then

$$
A_{t}\left(w_{l}^{T}\right) \leq \pi\left(\sum_{e \in \mathbb{E}^{+}} \ell(e)\right)^{2}
$$

Proof. The assumptions together with Lemma 13 yield that for any face $F \in \mathbb{F}_{b}$,

$$
\bar{n}_{w_{l}^{T}}(F) \leq d\left(F, F_{\infty}\right)
$$

Let us now choose a loop $\bar{l}$ as in Lemma 16. Then, $A_{t}\left(w_{l}^{T}\right) \leq A(\bar{l})$ and Banchoff-Pohl inequality applied to $\bar{l}$ yields the expected bound. 


\subsection{Asymptotics of Wilson loops as $N \rightarrow \infty \quad$ We shall consider the following families of loops.}

Definition 1. A skein is a finite multiset of loops of $L\left(\mathbb{R}^{2}\right)$. It is regular if its associated set is composed of distinct smooth loops, forming transverse intersections of multiplicity at most 2. A skein is affine if the corresponding set is a regular skein composed of piecewise affine loops. The set of skeins, regular and affine skeins are respectively denoted by $\operatorname{Sk}\left(\mathbb{R}^{2}\right), \operatorname{Sk}_{r}\left(\mathbb{R}^{2}\right)$ and $\mathcal{E}_{\mathcal{A}}$. For any $S \in \operatorname{Sk}\left(\mathbb{R}^{2}\right), \# S$ and $\ell(S)$ denote respectively the number of elements of $S$ counted with multiplicity and the sum of lengths counted without multiplicity.

We endow $\operatorname{Sk}\left(\mathbb{R}^{2}\right)$ with the quotient topology for the map $\coprod_{m \geq 1} \mathrm{~L}\left(\mathbb{R}^{2}\right)^{m} \rightarrow \operatorname{Sk}\left(\mathbb{R}^{2}\right)$, where for each $m \geq 1, \mathrm{~L}\left(\mathbb{R}^{2}\right)^{m}$ is endowed ${ }^{5}$ with the product topology. It is elementary to show that the spaces $\operatorname{Sk}_{r}\left(\mathbb{R}^{2}\right)$ and $\mathcal{E}_{\mathcal{A}}$ are dense in $\operatorname{Sk}\left(\mathbb{R}^{2}\right)$. If the multiset $\mathcal{S}=$ $\left\{l_{1}, \ldots, l_{m}\right\}$ is a skein, let us define for any $N \in \mathbb{N}^{*}$,

$$
\Phi_{N}(\mathcal{S})=N^{m-2} C_{m}\left(\operatorname{Tr}\left(H_{l_{1}}\right), \ldots, \operatorname{Tr}\left(H_{l_{m}}\right)\right),
$$

where the cumulants are with respect to the measure $\mathrm{YM}_{N}$. Observe that the law of the unitary Brownian motion is invariant under complex conjugation. Hence, for any skein $\mathcal{S}=\left\{l_{1}, \ldots, l_{m}\right\} \in \operatorname{Sk}\left(\mathbb{R}^{2}\right)$, denoting $\mathcal{S}^{*}=\left\{l_{1}^{-1}, \ldots, l_{m}^{-1}\right\}$,

$$
\Phi_{N}(\mathcal{S})=\overline{\Phi_{N}(\mathcal{S})}=\Phi_{N}\left(\mathcal{S}^{*}\right)
$$

is real-valued.

Proposition 4. For any affine skein $\mathcal{S} \in \mathcal{E}_{\mathcal{A}}$, the sequence $\Phi_{N}(\mathcal{S})$ converges as $N \rightarrow \infty$. We denote its limit by $\Phi(\mathcal{S})$.

Proof. For any affine skein $\mathcal{S}$, there exists an embedded graph $\mathbb{G}$ such that the elements of $\mathcal{S}$ belong to L $(\mathbb{G})$. Choosing an arbitrary base point $v \in \mathbb{V}$ and decomposing each loop in a lassos basis, yields that under $\mathrm{YM}_{N}$, the random family $\left(H_{l}\right)_{l \in \mathcal{S}}$ has the same law as a collection of words in marginals of independent $\mathrm{U}(N)$ Brownian motions. Therefore, the Proposition 1 implies the result.

Proposition 5. Let us fix a constant $K>0$. For any skein $\mathcal{S} \in \mathcal{E}_{\mathcal{A}}$ of loops of length smaller than $\frac{K}{3}>0$ and taking their values in a ball of radius $\frac{K}{3}$,

$$
\left|\Phi_{N}(\mathcal{S})-\Phi(\mathcal{S})\right| \leq \frac{\pi \# \mathcal{S}^{2} K^{2}}{N^{2}} e^{\pi \# \mathcal{S}^{2} K^{2}} .
$$

Proof. Let us assume that $\mathcal{S}=\left\{l_{1}, \ldots, l_{m}\right\}$ is a family of loops in $\mathcal{E}_{\mathcal{A}}$ all based at 0 . Let $\mathbb{G}_{\mathcal{S}}=\left(\mathbb{V}_{\mathcal{S}}, \mathbb{E}_{\mathcal{S}}, \mathbb{F}_{\mathcal{S}}\right)$ be the embedded graph with vertices the set of intersection points of the elements of $\mathcal{S}$ and with edges the restriction of elements of $\mathcal{S}$ between points of intersection. The loop $l_{1} l_{2} \ldots l_{n}$ satisfies the conditions of Lemma 17. Let us choose $T$ as in this Lemma and decompose each element of $\mathcal{S}$ in the corresponding lassos basis $\lambda^{T}$. Then, the second inequality of Lemma 7, for $k=1$ and the bound of Lemma 17 imply

$$
\left|\Phi_{N}(\mathcal{S})-\Phi(\mathcal{S})\right| \leq N^{-2} A_{t}\left(w_{l_{1} \ldots l_{m}}^{T}\right) e^{A_{t}\left(w_{l_{1} \ldots l_{m}}^{T}\right)}
$$

\footnotetext{
5 with this convention, the function $\ell: \operatorname{Sk}\left(\mathbb{R}^{2}\right) \rightarrow \mathbb{R}_{+}$is not continuous but upper continuous.
} 


$$
\leq \frac{\pi}{N^{2}}\left(\ell\left(l_{1}\right)+\cdots+\ell\left(l_{m}\right)\right)^{2} e^{\pi\left(\ell\left(l_{1}\right)+\cdots+\ell\left(l_{m}\right)\right)^{2}} .
$$

Consider now $\left\{l_{1}, \ldots, l_{m}\right\} \in \mathcal{E}_{\mathcal{A}}$ satisfying the assumption of the Proposition. For any $\epsilon>0$, let us choose piecewise affine paths $c_{1}, \gamma_{1}^{\epsilon}, \ldots, c_{m}, \gamma_{m}^{\epsilon}$, such that $\gamma_{i}^{\epsilon}=0=$ $\overline{c_{i}}, \overline{\gamma_{i}^{\epsilon}}=\underline{l_{i}}=\underline{c_{i}}, \ell\left(\gamma_{i}^{\epsilon}\right), \ell\left(c_{i}\right) \leq K(1+\epsilon)$, for any $i \in[m], \mathcal{S}^{\epsilon}=\left\{\gamma_{i}^{\epsilon} l_{i} c_{i}, \overline{i \in} \in[m]\right\}$ is an affine skein and $\gamma_{i}^{\epsilon} \rightarrow c_{i}^{-1}$, for any $i \in[m]$. Theorem 8 implies that $\Phi_{N}\left(\mathcal{S}^{\epsilon}\right) \rightarrow$ $\Phi_{N}\left(\left\{c_{i}^{-1} l_{i} c_{i}, i \in[m]\right\}\right)=\Phi_{N}(\mathcal{S})$. The application of the bound (39) for $\mathcal{S}^{\epsilon}$, uniform in $\epsilon$, implies that $\Phi_{N}(\mathcal{S})$ admits a limit $\Phi(\mathcal{S})$, as $N \rightarrow \infty$, and that the claimed bound holds true.

This result allows then to extend the function $\Phi$ to all of $\operatorname{Sk}\left(\mathbb{R}^{2}\right)$. Surprisingly, an argument analogue to the proof of Theorem 5.14. of [35] applies as well to the higher order case.

Theorem 9. For any skein $\mathcal{S} \in \operatorname{Sk}\left(\mathbb{R}^{2}\right)$, the sequence $\Phi_{N}(\mathcal{S})$ converges as $N \rightarrow \infty$. We denote its limit by $\Phi(\mathcal{S})$. The function $\Phi$ is a real-valued continuous function on $\operatorname{Sk}\left(\mathbb{R}^{2}\right)$. If $h$ is an area-preserving diffeomorphism of $\mathbb{R}^{2}$, for any $\mathcal{S} \in \operatorname{Sk}\left(\mathbb{R}^{2}\right), \Phi(h(\mathcal{S}))=$ $\Phi(\mathcal{S})$.

We shall also call the function $\Phi: \operatorname{Sk}\left(\mathbb{R}^{2}\right) \longrightarrow \mathbb{R}$ planar master field.

Proof. For any $K>0$, let $\mathrm{Sk}_{K}$ (respectively $\mathcal{E}_{K}$ ) be the set of skeins $\mathcal{S}$ (respectively affine skeins with distinct loops) with elements included in the ball of radius $r$ around 0 and with length less than $r$, with $r(\# \mathcal{S})^{2}=K$. As $\cup_{K>0} \mathrm{Sk}_{K}=\mathrm{Sk}\left(\mathbb{R}^{2}\right)$, it is enough to prove the result on $\mathrm{Sk}_{K}$. The set $\mathcal{E}_{K}$ is dense in $\mathrm{Sk}_{K}$. Indeed, any loop of $\mathrm{L}\left(\mathbb{R}^{2}\right)$ can be approximated by its linear interpolation, which itself can be approached by piecewise linear loops with simple intersections, without increasing its length. According to Theorem 8 , for any $N \geq 1$, the function $\Phi_{N}$ is continuous on $\mathrm{Sk}_{K}$. Moreover, Proposition 5 shows that $\Phi_{N}$ converges uniformly towards $\Phi$ on $\mathcal{E}_{K}$. Therefore, $\Phi_{N}$ converges uniformly on $\mathrm{Sk}_{K}$ towards the unique continuous extension $\tilde{\Phi}$ of $\Phi$ to $\mathrm{Sk}_{K}$.

A consequence of this Theorem is that for any $m \geq 3$, and any loops $l_{1}, \ldots, l_{m}$ in $\mathrm{L}\left(\mathbb{R}^{2}\right)$, under $\mathrm{YM}_{N}$,

$$
C_{m}\left(\operatorname{Tr}\left(H_{l_{1}}\right), \ldots, \operatorname{Tr}\left(H_{l_{m}}\right)\right)=N^{2-m} \Phi_{N}\left(l_{1}, \ldots, l_{m}\right) \rightarrow 0,
$$

as $N \rightarrow \infty$. The following theorem follows.

Theorem 10. Under $\mathrm{YM}_{N}$, the random family $\left(\operatorname{Tr}\left(H_{l}\right)-\mathbb{E}\left[\operatorname{Tr}\left(H_{l}\right)\right]\right)_{l \in \mathrm{L}\left(\mathbb{R}^{2}\right)}$ converges weakly as $N \rightarrow \infty$, towards a Gaussian field $\left(\phi_{l}\right)_{l \in \mathrm{L}\left(\mathbb{R}^{2}\right)}$, such that for any $a, b \in$ $\mathrm{L}\left(\mathbb{R}^{2}\right), \operatorname{cov}\left(\phi_{a}, \phi_{b}\right)=\Phi(\{a, b\})$. If $\left(l_{n}\right)_{n \geq 0}$ is a fixed sequence of loops in $\mathrm{L}\left(\mathbb{R}^{2}\right)$ that converges towards $l \in \mathrm{L}\left(\mathbb{R}^{2}\right)$, then $\phi_{l_{n}} \rightarrow \phi_{l}$ in distribution. If $h$ is an area preserving diffeomorphism of $\mathbb{R}^{2}$, the process $\left(\phi_{h(l)}\right)_{l \in \mathrm{L}\left(\mathbb{R}^{2}\right)}$ has the same law as $\left(\phi_{l}\right)_{l \in \mathrm{L}\left(\mathbb{R}^{2}\right)}$.

6.8. Yang-Mills measure with a polynomial potential. When $V$ is a function on $L\left(\mathbb{R}^{2}\right)$, let us set $\mathrm{L}_{V}=\left\{l \in \mathrm{L}\left(\mathbb{R}^{2}\right): V(l) \neq 0\right\}$ and $\operatorname{Sk}_{V}=\left\{\mathcal{S} \in \operatorname{Sk}\left(\mathbb{R}^{2}\right): \forall l \in \mathcal{S}, V(l) \neq 0,\right\}$. We shall consider the space $\mathcal{F}_{1}$ of functions $V$ such that $\mathrm{L}_{V}$ is countable and

$$
\|V\|_{1}=\sum_{l \in \mathrm{L}_{V}}|V(l)|<\infty .
$$


For any $V \in \mathcal{F}_{1}$ and $N \in \mathbb{N}^{*}$, under $\mathrm{YM}_{N}$, almost surely the following sum converges absolutely in operator norm and defines a random variable

$$
V_{N}=\sum_{l \in \mathrm{L}_{V}} V(l) H_{l}
$$

For any function $V \in \mathbb{C}^{\mathrm{L}\left(\mathbb{R}^{2}\right)}$, let us say that $V$ is symmetric if for any $l \in \mathrm{L}\left(\mathbb{R}^{2}\right)$, $V\left(l^{-1}\right)=\overline{V(l)}$. For any symmetric function $V \in \mathcal{F}_{1}$, the random matrix $V_{N}$ is Hermitian and its operator norm is bounded by $\|V\|_{1}$. In particular, $0<\mathbb{E}\left[e^{N \operatorname{Tr}\left(V_{N}\right)}\right]<\infty$. Let $\mathrm{YM}_{N, V}$ be the probability measure on $\left(\mathcal{M}_{N}\left(\mathrm{P}\left(\mathbb{R}^{2}\right)\right), \mathcal{C}\right)$, whose density with respect to $\mathrm{YM}_{N}$ is $\mathbb{E}\left[e^{N \operatorname{Tr}\left(V_{t, N}\right)}\right]^{-1} e^{N \operatorname{Tr}\left(V_{N}\right)}$. We shall denote by $\left(H_{l}^{V}\right)_{l \in \mathrm{L}\left(\mathbb{R}^{2}\right)}$ the canonical process on $\mathcal{M}_{N}\left(\mathrm{P}\left(\mathbb{R}^{2}\right)\right)$ with law $\mathrm{YM}_{N, V}$. When $\mathcal{S} \in \operatorname{Sk}\left(\mathbb{R}^{2}\right)$ has multiplicities $m_{1}, \ldots, m_{k}$, we set

$$
m_{\mathcal{S}}=m_{1} ! \ldots m_{k} !
$$

For any $V \in \mathbb{C}^{W_{q}}$ and $a \in \mathrm{L}\left(\mathbb{R}^{2}\right)$, let us define

$$
\mathcal{J}_{V}(a)=\sum_{\mathcal{S} \in \mathrm{Sk}_{V}} m_{\mathcal{S}}^{-1}\left(\pi \ell(\{a\} \cup \mathcal{S})^{2} \# \mathcal{S}\right)^{\# \mathcal{S}} \prod_{l \in \mathcal{S}}|V(l)|
$$

and for $a, b \in \mathrm{L}\left(\mathbb{R}^{2}\right)$,

$$
\mathcal{J}_{V}(a, b)=\sum_{\mathcal{S} \in \mathrm{Sk}_{V}} m_{\mathcal{S}}^{-1}\left(\pi \ell(\{a, b\} \cup \mathcal{S})^{2} \# \mathcal{S}\right)^{\# \mathcal{S}} \prod_{l \in \mathcal{S}}|V(l)| .
$$

Theorem 11. For any symmetric function $V \in \mathcal{F}_{1}$ and $l \in \mathrm{L}\left(\mathbb{R}^{2}\right)$ such that $\mathcal{J}_{V}(l)<\infty$, $\mathbb{E}_{\mathrm{YM}_{N, V}}\left(\frac{1}{N} \operatorname{Tr}\left(H_{l}\right)\right) \rightarrow \Phi_{V}(l)$, as $N \rightarrow \infty$, where

$$
\Phi_{V}(l)=\sum_{\mathcal{S} \in \mathrm{Sk}_{V}} \Phi(\{l\} \cup \mathcal{S}) m_{\mathcal{S}}^{-1} \prod_{l^{\prime} \in \mathcal{S}} V\left(l^{\prime}\right)
$$

is absolutely converging. Moreover,

$$
\operatorname{Var}_{\mathrm{YM}_{N, V}}\left(\frac{1}{N} \operatorname{Tr}\left(H_{l}\right)\right) \leq \frac{1}{N^{2}} \mathcal{J}_{V}(l, l) .
$$

Proof. For any affine skein $\mathcal{S} \in \mathcal{E}_{\mathcal{A}}$, with $m$ elements $l_{1}, \ldots, l_{m}$, let us choose an embedded graph $\mathbb{G}_{\mathcal{S}}$ with area's vector $t$, as in the proof of Proposition 5, for the affine skein without multiplicities associated to $\mathcal{S}$. Then, if $T$ is a spanning tree of $\mathbb{G}_{\mathcal{S}}$ satisfying the condition of Lemma 14, Lemma 17 implies that for any $l \in \mathcal{S},\left\|\bar{n}_{w_{l}^{T}}\right\|^{2} \leq \pi \ell(\mathcal{S})^{2}$. Then, for any Cayley tree $\mathfrak{T}_{t} \in \mathcal{C}_{m}, \mathfrak{T}_{t}\left(w_{l_{1}}^{T}, \ldots, w_{l_{m}}^{T}\right) \leq \pi^{m-1} \ell(\mathcal{S})^{2 m-2}$ (recall the definition (28) of the left hand side). Combining this inequality with Proposition 2, yields for every $N \in \mathbb{N}^{*}$,

$$
\left|\Phi_{N}(\mathcal{S})\right| \leq m^{m-2} \pi^{m-1} \ell(\mathcal{S})^{2 m-2} .
$$

By continuity of $\Phi_{N}$, it follows that this inequality holds true for all skein $\mathcal{S} \in \operatorname{Sk}\left(\mathbb{R}^{2}\right)$. Let us fix a symmetric function $V \in \mathcal{F}_{1}$ on $\mathrm{L}\left(\mathbb{R}^{2}\right)$ and $l \in \mathrm{L}\left(\mathbb{R}^{2}\right)$ such that $\mathcal{J}_{V}(l)<\infty$. For every $N \in \mathbb{N}^{*}$, the function $x \in \mathbb{R} \mapsto \mathbb{E}\left(\exp \left(x N \operatorname{Tr}\left(V_{N}\right)\right)\right)$ is analytic. According to the previous inequality and to the assumption on $V$, its Taylor expansion at 0 has a radius bigger than one and equals 


$$
\sum_{\substack{m \geq 0 \\ l_{1}, \ldots, l_{m} \in \mathrm{L}\left(\mathbb{R}^{2}\right)}} \frac{1}{m !} x^{m} \prod_{i=1}^{m} V\left(l_{i}\right) \Phi_{N}\left(l, l_{1}, \ldots, l_{m}\right)=\sum_{\mathcal{S} \in \operatorname{Sk}_{V}} \frac{x^{\# \mathcal{S}}}{m_{\mathcal{S}}} \prod_{l \in \mathcal{S}} V(l) \Phi_{N}(\{l\} \cup \mathcal{S}) .
$$

Each term of the sum over $m$ being uniformly bounded in $N$, by dominated convergence, Theorem 9 yields the first result. The bound on the variance follows by a similar argument, using equality (4).

Remark 5. For any $V \in \mathcal{F}_{1}$, let us write $\|V\|_{\infty}=\sup _{l \in \mathrm{L}\left(\mathbb{R}^{2}\right)}|V(l)|$ and $\ell(V)=$ $\sup _{\mathcal{S} \in \mathrm{Sk}_{V}} \ell(\mathcal{S})$. For any $V \in \mathcal{F}_{1}$ and $l \in \mathrm{L}\left(\mathbb{R}^{2}\right)$, if $(\ell(V)+\ell(l))^{2}<\frac{1}{e \pi\|V\|_{\infty}}$, then $\mathcal{J}_{V}(l)<\infty$.

6.9. Small area limit. For any $\alpha>0$ and any loop $l \in \mathrm{L}\left(\mathbb{R}^{2}\right)$, denote by $\alpha . l$ the image of $l$ by the dilatation of rate $\alpha$, centered at 0 . If $\mathcal{S}=\left\{l_{1}, \ldots, l_{m}\right\}$ is a skein, $\alpha . \mathcal{S}=$ $\left\{\alpha . l_{1}, \ldots, \alpha . l_{m}\right\}$. The following proposition shows that, as $\alpha \rightarrow 0$, all the quantities defined above have the same behavior as $\alpha \rightarrow 0$.

Proposition 6. The following Taylor expansions are true for any $N \in \mathbb{N}^{*}$. As $\alpha \rightarrow 0$, for any loop $l \in \mathrm{L}\left(\mathbb{R}^{2}\right)$,

$$
\Phi_{N}(\sqrt{\alpha} . l)=1-\frac{\alpha}{2} \int_{\mathbb{R}^{2}} n_{l}^{2}(x) d x+O\left(\alpha^{2}\right)=\Phi(\sqrt{\alpha} . l)+O\left(\alpha^{2}\right)
$$

and for any skein $\mathcal{S}$ with at least two loops,

$$
\begin{aligned}
\Phi_{N}(\sqrt{\alpha} \cdot \mathcal{S}) & =(-\alpha)^{\# \mathcal{S}-1} \sum_{\mathfrak{T}_{\mathcal{S}}} \prod_{\left\{l_{1}, l_{2}\right\} \in \mathfrak{T}_{\mathcal{S}}} \int_{\mathbb{R}^{2}} n_{l_{1}}(x) n_{l_{2}}(x) d x+O\left(\alpha^{\# \mathcal{S}}\right) \\
& =\Phi(\sqrt{\alpha} \cdot \mathcal{S})+O\left(\alpha^{\# \mathcal{S}}\right),
\end{aligned}
$$

where the sum is over connected graph with vertices $\mathcal{S}$ and $\# \mathcal{S}-1$ edges. In both cases, there exists a positive continuous function $b$, independent of $N$, such that $O\left(\alpha^{|\mathcal{S}|}\right) \leq$ $\alpha^{|\mathcal{S}|} b\left(\sum_{l \in \mathcal{S}} \ell(l)\right)$.

Proof. If $\mathcal{S} \in \mathcal{E}_{\mathcal{A}}$, the assertion is a direct consequence of Proposition 2 and (37). Continuity of the functions $\Phi_{N}, \Phi$ and $b$ allows then to conclude.

A direct consequence is the following

Corollary 2. Let $W$ be a white noise on $\mathbb{R}^{2}$, with intensity given by the Lebesgue measure.

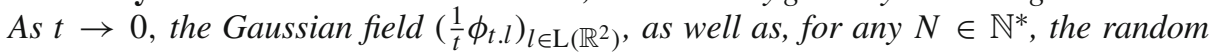
family $\left(t^{-1}\left(\operatorname{Tr}\left(H_{t . l}\right)-N \Phi(t . l)\right)\right)_{l \in \mathrm{L}\left(\mathbb{R}^{2}\right)}$, under $\mathrm{YM}_{N}$, converge in distribution towards the Gaussian field $\left(i W\left(n_{l}\right)\right)_{l \in \mathrm{L}\left(\mathbb{R}^{2}\right)}$.

\section{Makeenko-Migdal Equations}

We shall now address the problem of the computation and characterization of the master field. Let $\overline{\mathrm{Sk}_{r}}\left(\mathbb{R}^{2}\right)$ be the quotient of $\mathrm{Sk}_{r}\left(\mathbb{R}^{2}\right)$ under the action of diffeomorphisms of the plane. For any integer $n$, the set of equivalence classes of skeins with less than $n$ intersections is finite. Thanks to its invariance property under area-preserving diffeomorphisms and to its continuity, the master field is characterized by its value on $\mathrm{Sk}_{r}\left(\mathbb{R}^{2}\right)$ and yields functions indexed by $\overline{\mathrm{Sk}_{r}}\left(\mathbb{R}^{2}\right)$ that can be expressed inductively. 


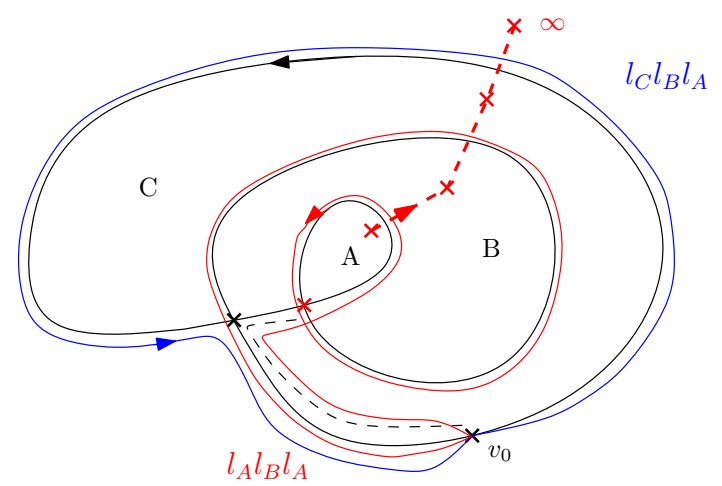

Fig. 4. An example with a loop winding three times around the origin and the decomposition of two loops traced in its image. The spanning tree associated to the free basis and its dual are drawn in dashed lines

7.1. Makeenko-Migdal equations for the master field on skeins. For any skein $\mathcal{S}$, let us denote by $W_{\mathcal{S}}$ the expectation $\mathbb{E}_{\mathrm{YM}_{N}}\left[\prod_{l \in \mathcal{S}} \operatorname{Tr}\left(H_{l}\right)\right]$, we call this function a Wilson $\operatorname{skein}^{6}$ and say it is regular whenever the associated skein is. In view of the definition of discrete Yang-Mills measure, one may try to compute the master field of higher order of a regular skein $\mathcal{S}$ using Itô formula to yield a first order differential system for the

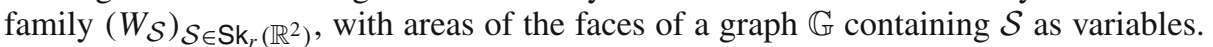
However, this differential system yields at first sight non-regular Wilson skeins $W_{\mathcal{S}}$ as features the Example 1.

Example 1. Consider a loop $l$ that winds three times around the origin. Let us name the faces $A, B$ and $C$ and choose a lassos basis $\left(l_{A}, l_{B}, l_{C}\right)$ according to a spanning tree as illustrated in Fig. 4 in dashed lines. In this basis, the loop is decomposed as $l=l_{C} l_{B} l_{A}^{2} l_{B} l_{A}$.

Using Itô formula as described in Lemma 4 and differentiating with respect to the area of the faces $C$ and $B$ yields $\frac{d}{d|C|} W_{l}=-\frac{W_{l}}{2}$ and

$$
N\left(\frac{d}{d|B|}\left(W_{l}\right)+W_{l}\right)=-W_{\left\{l_{B} l_{A}^{2}, l_{C} l_{B} l_{A}\right\}}=-W_{\left\{l_{A} l_{B} l_{A}, l_{C} l_{B} l_{A}\right\}} .
$$

These first two derivatives can be expressed in terms of regular Wilson skeins. However, the derivative with respect to the face of index 3 yields terms that do not seem to be polynomials of regular Wilson skeins:

$$
N\left(\frac{d}{d|A|}\left(W_{l}\right)+\frac{3}{2} W_{l}\right)=-W_{\left\{l_{B} l_{A}, l_{C} l_{B} l_{A}^{2}\right\}}-W_{\left\{l_{C} l_{B} l_{A}, l_{A} l_{B} l_{A}\right\}}-W_{\left\{l_{A}, l_{B} l_{A} l_{C} l_{B} l_{A}\right\}} .
$$

For any regular skein $\mathcal{S}$, one must therefore face the problem of finding a closed system of Wilson skeins containing $W_{\mathcal{S}}$. The system of equations given by Lemma 4 gives such a system, but its size happens to grow exponentially with the number of faces of the original skein (see section 6.8 of [35], where the smallest closed system obtained is made of what is called therein Wilson garlands). The Makeenko-Migdal equation solves this problem and gives linear combinations of area derivatives operators that preserve the

\footnotetext{
6 We warn the Reader that these functions are not normalized as they can be in the literature, so that, with this convention, $W_{\mathrm{cst}}=N$.
} 


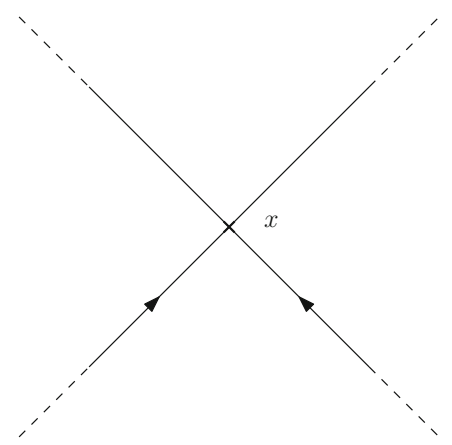

$\mathcal{S}$

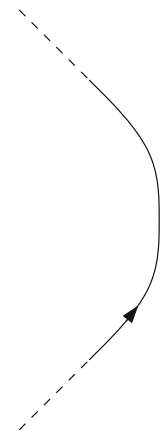

$\mathcal{S}_{x}$

Fig. 5. Local transformation at an intersection point $x$ of a skein $\mathcal{S}$

set of function indexed by skeins, so that the size of the system grows as a polynomial in the number of faces. Let $\mathcal{S}$ be a regular skein and $x$ be a point of intersection of its elements (between themselves or each other). Let us denote by $\mathcal{S}_{x}$ the skein composed with the same loops as $\mathcal{S}$ except for the loop or the pair of loops containing $x$ that is replaced respectively by the pair of loops or the loop based at $x$, which instead of going straight along the same strand of $\mathcal{S}$, turns at the point $x$ using the other outgoing strand (see Fig. 5).

The following proposition is proved in [35], in a more general framework ${ }^{7}$ and relies on integration by parts applied to the product of a function on $\mathcal{M}_{N}(\mathrm{P}(\mathbb{G}))$ with the density of the discrete Yang-Mills measure. We provide here another proof relying on the decomposition in lassos described in Sect. 6.6 and on the invariance of the Brownian motion by adjunction. Let us fix a regular skein $\mathcal{S}$ and an embedded graph $\mathbb{G}$, such that elements of $\mathcal{S}$ belong to $\mathrm{P}(\mathbb{G})$.

Proposition 7 (Makeenko-Migdal equation). Let $F_{1}, \ldots, F_{4}$ be the four faces of $\mathbb{G}$ around a point of intersection $x \in \mathbb{V}$ of $\mathcal{S}$ in a cyclic order and such that $F_{1}$ is the face bounded by the two incoming edges of $\mathcal{S}$ at $x$. If their area are respectively parametrized by $t_{1}, \ldots, t_{4}$, then,

$$
\left(\frac{d}{d t_{1}}-\frac{d}{d t_{2}}+\frac{d}{d t_{3}}-\frac{d}{d t_{4}}\right) \mathbb{E}_{\mathrm{YM}_{N}}\left[\prod_{l \in \mathcal{S}} \operatorname{Tr}\left(H_{l}\right)\right]=\frac{1}{N} \mathbb{E}_{\mathrm{YM}_{N}}\left[\prod_{l \in \mathcal{S}_{x}} \operatorname{Tr}\left(H_{l}\right)\right] .
$$

In the latter left hand side, when two faces $F_{i}, F_{j}$ agree or if $F_{k}$ is unbounded, then by convention $\frac{d}{d t_{i}}=\frac{d}{d t_{j}}$ or $\frac{d}{d t_{k}}=0$. Using this convention, we denote by $\mu_{x}$ the operator $\frac{d}{d t_{1}}-\frac{d}{d t_{2}}+\frac{d}{d t_{3}}-\frac{d}{d t_{4}}$, where the faces are numbered as in the Proposition. For any skein $\mathcal{S}$, let us set $n_{\mathcal{S}}=\sum_{l \in \mathcal{S}} n_{l}$. Notice that for $N=1$, the equality (40) is equivalent to the fact

$$
n_{\mathcal{S}}\left(F_{1}\right)^{2}-n_{\mathcal{S}}\left(F_{2}\right)^{2}+n_{\mathcal{S}}\left(F_{3}\right)^{2}-n_{\mathcal{S}}\left(F_{4}\right)^{2}=-2 .
$$

The strategy of our proof is to choose an embedded graph $\mathbb{G}$ containing $\mathcal{S}$ and an appropriate basis of $\mathrm{RL}_{x}(\mathbb{G})$, so that using properties of Sect. 6.6 and Lemma 6 , terms on

\footnotetext{
${ }^{7}$ For intersections of higher degree and for classical compact Lie groups.
} 
the left-hand-side of (40) cancel themselves leaving a single cut and join transformation. Such a cancellation appears in the following situation. Let us recall notation of Sect. 3.2.3 and set for any words $w \in W_{q}^{m}$, and $t \in \mathbb{R}_{+}^{q}, E_{t}(w)=K_{t}\left(w, 1_{m}\right)$.

Lemma 18. For $q \geq 4$, let $w_{1}, \ldots, w_{m}$ be $m$ words such that

$$
w_{i}=\mathfrak{m}_{i}\left(x_{1} x_{2} x_{3} x_{4}, x_{2} x_{3}, x_{3} x_{4}, x_{5}, \ldots, x_{q}\right),
$$

with $\mathfrak{m}_{i} \in W_{q-1}$. Let us assume that

$$
\bar{n}_{\mathfrak{m}}(2)=\bar{n}_{\mathfrak{m}}(3)=1,
$$

where $\mathfrak{m}=\mathfrak{m}_{1} \ldots \mathfrak{m}_{m}$. If $y^{\epsilon_{a}}, y^{\epsilon_{b}}$ occur in position a and $b$ in $\tilde{w}_{1} \ldots \tilde{w}_{m}$, where $\tilde{w}_{i}=$ $\mathfrak{m}_{i}\left(x_{1} x_{2} x_{3} x_{4}, x_{2} y, y x_{4}, x_{5}, \ldots, x_{q}\right)$, for $i \in[m]$, then

$$
\left(\frac{d}{d t_{1}}-\frac{d}{d t_{2}}+\frac{d}{d t_{3}}-\frac{d}{d t_{4}}\right) E_{t}\left(w_{1}, \ldots, w_{m}\right)=-\frac{\epsilon_{a} \epsilon_{b}}{N} E_{t}\left(\mathcal{T}_{a, b}\left(w_{1}, \ldots, w_{m}\right)\right) .
$$

Proof. Let $A$ be a subset of $[\ell(w)]$ such that the restriction of $w=w_{1} \ldots w_{m}$ to $A$ is of the form $v_{1}\left(x_{i} x_{j}\right) \ldots v_{m}\left(x_{i} x_{j}\right)$, with for any $p \in[m], v_{p} \in W_{1}$ and $v_{p}\left(x_{i} x_{j}\right)$ is a restriction of $w_{p}$. Then, an inspection of the definition of Sect. 3.2.2 yields that for any $\epsilon \in\{-1,1\}, \mathcal{N}_{w}^{\epsilon}(j) \cap A^{2}=(\epsilon, \epsilon)+\mathcal{N}_{w}^{\epsilon}(i) \cap A^{2}$ and

$$
\mathcal{T}_{p, q}\left(w_{1}, \ldots, w_{m}\right)=\mathcal{T}_{p+\epsilon, q+\epsilon}\left(w_{1}, \ldots, w_{m}\right) .
$$

Let $A, B, C$ be the set of occurrences of $x, y, z$ in $\hat{w}_{1} \ldots \hat{w}_{m}$, where for each $i \in[m]$, $\hat{w}_{i}=\mathfrak{m}_{i}\left(x^{4}, y^{2}, z^{2}, x_{5}, \ldots, x_{q-1}\right)$. According to Lemma 6 and (42),

$$
\begin{aligned}
\left(\frac{d}{d t_{1}}-\frac{d}{d t_{2}}+\frac{d}{d t_{3}}-\frac{d}{d t_{4}}\right) E_{t}(\mathbf{w})= & \frac{\bar{n}_{w}(4)-\bar{n}_{w}(3)+\bar{n}_{w}(2)-\bar{n}_{w}(1)}{2} E_{t}(\mathbf{w}) \\
& -\frac{1}{N} \sum \epsilon E_{t}\left(\mathcal{T}_{p, q} \mathbf{w}\right)
\end{aligned}
$$

where the sum is over $\epsilon \in\{-1,1\}$ and $(p, q) \in \mathcal{N}_{w}^{\epsilon}(3) \cap(B \times C)$. By assumption the alternated sum of the right hand side vanishes and $\mathcal{N}_{w}^{\epsilon}(3) \cap(B \times C)$ equals $\{(a, b)\}$ if $\epsilon=\epsilon_{a} \epsilon_{b}$ and the empty set otherwise.

Definition 2. We say that two skeins $\mathcal{S}=\left\{l_{1}, \ldots, l_{m}\right\}, \mathcal{S}^{\prime}=\left\{l_{1}^{\prime}, \ldots, l_{m}^{\prime}\right\}$ are conjugated and write $\mathcal{S} \equiv \mathcal{S}^{\prime}$, if there exist $\gamma_{1}, \ldots, \gamma_{m} \in \mathrm{P}\left(\mathbb{R}^{2}\right)$ with $\underline{\gamma}_{i}=\underline{l}_{i}^{\prime}, \bar{\gamma}_{i}=\underline{l}_{i}$ and $l_{i}^{\prime}=\gamma_{i} l_{i} \gamma_{i}^{-1}$, for any $i \in[m]$.

Lemma 19. Let $x$ be a point of intersection of a regular skein $\mathcal{S}$, whose loops are all based at $y \neq x$. There exists an embedded graph $\mathbb{G}^{\prime}$, with $\mathrm{P}(\mathbb{G}) \subset \mathrm{P}\left(\mathbb{G}^{\prime}\right)$, a lassos basis $^{8} \Lambda$ of $\mathrm{RL}_{y}\left(\mathbb{G}^{\prime}\right)$ and a labeling of faces of $\mathbb{G}^{\prime}$ matching the condition of Proposition 7 , such that a decomposition of elements of $\mathcal{S}$, into words $w_{1}, \ldots, w_{m}$ in $\Lambda$, satisfies the condition of Lemma 18, with moreover $\epsilon_{a} \epsilon_{b}=-1$ and

$$
\left\{\tilde{w}_{i}(\lambda, \lambda \in \Lambda): 1 \leq i \leq m^{\prime}\right\} \equiv \mathcal{S}_{x}
$$

where $\left(\tilde{w}_{1}, \ldots, \tilde{w}_{m^{\prime}}\right)=\mathcal{T}_{a, b}\left(w_{1}, \ldots, w_{m}\right)$.

\footnotetext{
8 As defined in section 6.3.2.
} 


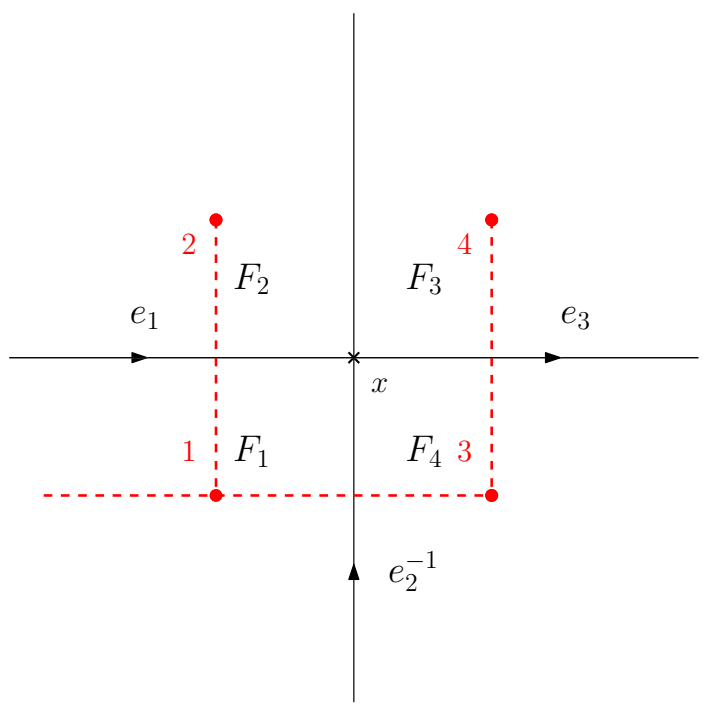

Fig. 6. A spanning tree $\hat{T}$ of $\hat{\mathbb{G}}^{\prime}$, such that $\hat{T}_{F_{1}}$ is a tree with vertices $\left\{F_{i}, i \in[4]\right\}$, with $F_{2}$ and $F_{3}$ as leaves. The order induced by the clockwise contour is denoted in red (color figure online)

Proof (Proposition 7). Let us choose a graph $\mathbb{G}^{\prime}$ and a lassos basis $\Lambda=\left(\lambda_{F}\right)_{F \in \mathbb{F}_{b}^{\prime}}$ according to Lemma 19. Then, according to Lemma 10 and Theorem 8 , under $\mathrm{YM}_{N}$, $\left(H_{\lambda}\right)_{F \in \mathbb{F}_{b}^{\prime}}$ has the same law as $\left(U_{F,|F|}\right)_{F \in \mathbb{F}_{b}^{\prime}}$, where $\left(U_{F}\right)_{F \in \in \mathbb{F}_{b}^{\prime}}$ are $\# \mathbb{F}_{b}^{\prime}$ independent $\mathrm{U}(N)$-Brownian motions. Let $t \in \mathbb{R}_{+}^{\mathbb{F}_{b}^{\prime}}$ be the vector of faces area of $\mathbb{G}^{\prime}$. For any skein $\tilde{\mathcal{S}}$, with elements in $\mathrm{L}\left(\mathbb{G}^{\prime}\right)$, let $\left\{\gamma_{l}, l \in \tilde{\mathcal{S}}\right\}$ be a family of paths of $\mathrm{P}\left(\mathbb{G}^{\prime}\right)$, with $\underline{\gamma}_{l}=y$ and $\bar{\gamma}_{l}=\underline{l}$ and denote by $\left(w_{1}, \ldots, w_{m}\right)$ a decomposition of $\tilde{\mathcal{S}}^{\prime}=\left\{\gamma_{l} l \gamma_{l}^{-1}, l \in \mathcal{\mathcal { S }}\right\}$ in the basis $\Lambda$. Then, as $H \in \mathcal{M}_{N}\left(\mathrm{P}\left(\mathbb{R}^{2}\right)\right)$,

$$
\mathbb{E}\left[\prod_{l \in \tilde{\mathcal{S}}} \operatorname{Tr}\left(H_{l}\right)\right]=\mathbb{E}\left[\prod_{l \in \tilde{\mathcal{S}}^{\prime}} \operatorname{Tr}\left(H_{l}\right)\right]=E_{t}\left(w_{1}, \ldots, w_{m}\right)
$$

and $\left(\frac{d}{d t_{1}}-\frac{d}{d t_{2}}+\frac{d}{d t_{3}}-\frac{d}{d t_{4}}\right) E_{t}\left(w_{1}, \ldots, w_{m}\right)=\mu_{x} \mathbb{E}\left[\prod_{l \in \mathcal{S}} \operatorname{Tr}\left(H_{l}\right)\right]$. Applying Lemma 18 and the former equality to $\mathcal{S}_{x}$ implies the claim.

Proof (Lemma 19). Let us consider an embedded graph $\mathbb{G}^{\prime}$ with $\mathrm{P}\left(\mathbb{G}^{\prime}\right) \supset \mathrm{P}(\mathbb{G})$, with a vertex $y \in \mathbb{V}^{\prime} \backslash\{x\}$ and labeled faces, such that the faces $F_{1}, \ldots, F_{4}$ are neighboring $x$ in $\mathbb{G}^{\prime}$, bounded, distinct, in clockwise order, with $F_{1}$ bounding the two ingoing edges and such that the graph $\left(\hat{\mathbb{V}}^{\prime} \backslash\left\{F_{1}, \ldots, F_{4}\right\}, \mathbb{E}^{\prime} \backslash \cup_{i=1}^{4}\left\{e, F_{i} \in e\right\}\right)$ is connected.

Such a graph can be obtained by splitting successively the faces of $\mathbb{G}$. Let us choose a spanning tree ${ }^{9} \hat{T}$ of $\hat{\mathbb{G}}^{\prime}$, such that the tree $\hat{T}_{F_{1}}$ has vertices $\left\{F_{i}, i \in[4]\right\}$ and edges $\left\{\left(F_{2}, F_{1}\right),\left(F_{3}, F_{4}\right),\left(F_{4}, F_{1}\right)\right\}$, see Fig. 6 . We denote by $T$ the spanning tree of $\mathbb{G}^{\prime}$ dual to $\hat{T}$. Let us consider the basis of lassos $\Lambda^{T}$ rooted at $y$, as defined in Sect. 6.6. Let us fix an ordering $\left(l_{1}, \ldots, l_{m}\right)$ of $\mathcal{S}$. According to (35) and (36), each element $l_{i}$ is decomposed into $\tilde{w}_{i}\left(\lambda_{F_{i}}, i \in[q]\right)$, with $q=\# \mathbb{F}_{b}^{\prime}$,

$$
\tilde{w}_{i}=\tilde{\mathfrak{m}}_{i}\left(x_{1} x_{2} x_{4} x_{3}, x_{2} x_{3}, x_{4} x_{3}, x_{5}, \ldots, x_{q}\right)
$$

\footnotetext{
${ }^{9}$ Recall the notation below Lemma 12.
} 
and $\tilde{\mathfrak{m}}_{l} \in W_{q-1}$. Moreover, if $\tilde{\mathfrak{m}}=\tilde{\mathfrak{m}}_{1} \ldots \tilde{\mathfrak{m}}_{m}$, as $F_{2}$ and $F_{3}$ are leaves of $\hat{T}, \bar{n}_{\tilde{\mathfrak{m}}}(2)=$ $\bar{n}_{\tilde{\mathfrak{m}}}(3)=1$. Let us emphasize that as $y \neq x, x_{2}$ and $x_{3}$ occur consecutively in $\tilde{w}_{i}$ only once, for $i_{0}$ such that $l_{i_{0}}$ goes through the edges dual to $\left(F_{2}, F_{1}\right)$ and $\left(F_{3}, F_{4}\right)$. Let us denote by $\Theta_{3,4}$ the automorphism of the free group $\mathrm{RL}_{x}\left(\mathbb{G}^{\prime}\right)$, that maps $\lambda_{F_{4}}$ to $\lambda_{F_{3}}^{-1} \lambda_{F_{4}} \lambda_{F_{3}}$ and fixes $\lambda_{F}$ for $F \neq F_{4}$. Then, the family $\mathcal{S}$ admits a decomposition $\left(w_{1}, \ldots, w_{m}\right)$ into the lassos basis $\Theta_{3,4}\left(\Lambda^{T}\right)$ satisfying the condition of Lemma 18. As the skein crosses the edge $\left(F_{3}, F_{4}\right)$ (directed towards $\left.F_{\infty}, \mathbb{G}^{\prime}\right)$ from right to left, whereas it crosses $\left(F_{4}, F_{1}\right)$ from left to right, $\epsilon_{a} \epsilon_{b}=-1$. To conclude, it remains to identify the right hand side of Lemma 18 as a decomposition of a skein in $\Theta_{3,4}\left(\Lambda^{T}\right)$. We shall only detail the case when $x$ is a point of self-intersection of $\mathcal{S}$, the case of the intersection of two different loops being similar. Let $l_{i_{0}}$ be the loop of $\mathcal{S}$ with an intersection point at $x$ and consider the three edges $e_{1}, e_{2}, e_{3}$ of $\mathbb{G}^{\prime}$ around $x$, crossing $\hat{T}$ from right to left, with the counterclockwise order (see Fig. 6). The decomposition of $l_{i_{0}}$ in the basis $\beta$ (recall (35)) is

$$
l_{i_{0}}=X \beta_{e_{1}} \beta_{e_{3}} Y \beta_{e_{2}}^{-1} Z \text { or } X \beta_{e_{2}}^{-1} Y \beta_{e_{1}} \beta_{e_{3}} Z,
$$

where $X, Y, Z$ are words in $\left\{\beta_{e}: e \in \mathbb{E} \backslash\left(T \cup\left\{e_{1}, e_{2}, e_{3}\right\}\right)\right\}$, so that

$$
w_{i_{0}}=W_{X} x_{2} x_{3} W_{Y} x_{4}^{-1} x_{3}^{-1} W_{Z} \text { or } W_{X} x_{4}^{-1} x_{3}^{-1} W_{Y} x_{2} x_{3} W_{Z},
$$

where $W_{X}, W_{Y}, W_{Z}$ are words of the form $m\left(x_{1} x_{2} x_{4} x_{3}, x_{5}, \ldots, x_{q}\right)$ for some $m \in$ $W_{q-3}$. Then, $\mathcal{T}_{a, b}^{-} \mathbf{w}$ is a permutation of $\left(w_{1}, \ldots, \hat{w}_{i_{0}}, \ldots, w_{m}, w_{l}, w_{r}\right)$, with

$$
w_{l}=W_{X} x_{2} W_{Z}, w_{r}=x_{3} W_{Y} x_{4}^{-1} x_{3}^{-1} \text { or } w_{l}=W_{Y} x_{2}, w_{r}=W_{X} x_{4}^{-1} x_{3}^{-1} x_{3} W_{Z} .
$$

In the first case, $w_{l}$ is the decomposition into $\Theta_{3,4}\left(\Lambda^{T}\right)$ of the loop rooted at $y$ following the strands of $l_{i_{0}}$ but at $x$, where it turns left, whereas $w_{r}$ is the decomposition of $\left[y, \underline{e_{3}}\right]_{T} l_{x}^{R}\left[\underline{e_{3}}, y\right]_{T}$, where $l_{x}^{R}$ is the loop based at $x$, using the strands of $l_{i_{0}}$ starting with $e_{3}$, until it uses $e_{2}^{-1}$. The second case is similar.

For any regular skein $\mathcal{S} \in \mathrm{Sk}_{r}\left(\mathbb{R}^{2}\right)$, let us denote respectively by $\mathbb{V}_{S}(\mathcal{S})$ and $\mathbb{V} f(\mathcal{S})$, or simply $\mathbb{V}_{s}, \mathbb{V}_{f}$, the set of self-intersection points of each loops and the set of intersection points of pair of distinct loops of $\mathcal{S}$. If $x \in \mathbb{V}_{S}$ is a point of intersection of one loop $l \in \mathcal{S}$, we denote respectively by $l_{x}^{L}$ and $l_{x}^{R}$ the loops based at $x$, that use respectively the left and right outgoing edges, following the strand of $l$ until their first return to $x$. If $x \in \mathbb{V}_{f}$ is the intersection point of two different loops $l_{1}$ and $l_{2}$ of $\mathcal{S}$, we denote by $l_{1} \circ_{x} l_{2}$ the concatenation of the loops obtained by rooting $l_{1}$ and $l_{2}$ at the point $x$.

Theorem 12. Let $\mathcal{S}=\left\{l_{1}, \ldots, l_{m}\right\}$ be a regular skein, $x \in \mathbb{V}_{\mathcal{S}}$ a point of intersection and $F_{1}, \ldots, F_{4}$ faces around $x$, with areas parametrized by $t_{1}, \ldots, t_{4}$, as in Proposition 7. If $x$ is the intersection point of two different loops $l_{1}$ and $l_{2}$,

$$
\left(\frac{d}{d t_{1}}-\frac{d}{d t_{2}}+\frac{d}{d t_{3}}-\frac{d}{d t_{4}}\right) \Phi_{N}(\mathcal{S})=\Phi_{N}\left(l_{1} \circ_{x} l_{2}, l_{3}, \ldots, l_{m}\right) .
$$

If $x$ is an intersection point of the loop $l \in \mathcal{S}$, then $\left(\frac{d}{d t_{1}}-\frac{d}{d t_{2}}+\frac{d}{d t_{3}}-\frac{d}{d t_{4}}\right) \Phi_{N}(\mathcal{S})$ equals

$$
\sum_{\substack{\mathcal{S}_{x}^{L} \sqcup \mathcal{S}_{x}^{R}=\mathcal{S}_{x} \\ l_{x}^{L} \in \mathcal{S}_{x}^{L} \text { and } l_{x}^{R} \in \mathcal{S}_{x}^{R}}} \Phi_{N}\left(\mathcal{S}_{x}^{L}\right) \Phi_{N}\left(\mathcal{S}_{x}^{R}\right)+\frac{1}{N^{2}} \Phi_{N}\left(l_{x}^{L}, l_{x}^{R}, l_{2}, \ldots, l_{m}\right) .
$$


Moreover, if $F \in \mathbb{F}_{b}$ is a neighbor face of the unbounded face, then

$$
\frac{d}{d|F|} \Phi_{N}(\mathcal{S})=-\frac{1}{2} \Phi_{N}(\mathcal{S})
$$

Proof. For any loops $l_{1}, \ldots, l_{m} \in \mathrm{L}\left(\mathbb{R}^{2}\right)$ and $\pi, \mathcal{V} \in \mathcal{P}_{m}$ with $\pi \leq \mathcal{V}$, let us set $\mathbb{E}_{\pi}\left[l_{1}, \ldots, l_{m}\right]=\prod_{B \in \pi} \mathbb{E}\left[\prod_{i \in B} \operatorname{Tr}\left(H_{l_{i}}\right)\right], C_{\mathcal{V}}\left[l_{1}, \ldots, l_{m}\right]=\prod_{B \in \mathcal{V}} \overline{C_{\# B}}\left(\operatorname{Tr}\left(H_{l_{i}}\right), i \in\right.$ $B$ ) and $C_{\pi, \mathcal{V}}\left[l_{1}, \ldots, l_{m}\right]=C_{\pi, \mathcal{V}}\left(\operatorname{Tr}\left(H_{l_{i}}\right), i \in[m]\right)$. Let us recall (38) and consider the normalized cumulant

$$
\Phi_{\pi}\left(l_{1}, \ldots, l_{m}\right)=\prod_{B \in \pi} \Phi\left(l_{i}, i \in B\right)=N^{m-2 \# \pi} C_{\pi}\left(l_{1}, \ldots, l_{m}\right) .
$$

Assume that $x$ is an intersection point of $l_{1}$ and denote by $\overline{\{1,2\}}$ the smallest partition of $[m+1]$ containing $\{1,2\}$. For any partition $\mathcal{V}$ of $[m+1]$ connecting 1 with 2 , denoting by $\mathcal{V}^{\prime}$ the partition of $[m]$ obtained by identifying 2 with 1 ,

$$
\begin{aligned}
\sum_{\{1,2\} \leq \pi \leq \mathcal{V}} N \mu_{x} C_{\pi^{\prime}}\left(l_{1}, l_{2}, \ldots, l_{m}\right) & =\sum_{\pi \leq \mathcal{V}^{\prime}} N \mu_{x} C_{\pi}\left(l_{1}, l_{2}, \ldots, l_{m}\right) \\
& =N \mu_{x} \mathbb{E}_{\mathcal{V}^{\prime}}\left[l_{1}, l_{2}, \ldots, l_{m}\right]=\mathbb{E}_{\mathcal{V}^{\prime}}\left[l_{1, x}^{L} l_{1, x}^{R}, l_{2}, \ldots, l_{m}\right] \\
& =\mathbb{E}_{\mathcal{V}}\left[l_{1, x}^{L}, l_{1, x}^{R}, l_{2}, \ldots, l_{m}\right] .
\end{aligned}
$$

Therefore, for any partition $\pi \in \mathcal{P}_{m+1}$ connecting 1 and 2 ,

$$
C_{\overline{\{1,2\}}, \pi}\left(l_{1, x}^{L}, l_{1, x}^{R}, \ldots, l_{m}\right)=N \mu_{x} C_{\pi^{\prime}}\left(l_{1}, l_{2}, \ldots, l_{m}\right) .
$$

In particular, according to the Leonov Schiryaev formula (5),

$$
\begin{aligned}
& \mu_{x} \Phi_{N}\left(l_{1}, \ldots, l_{m}\right)=N^{m-3} C_{\overline{\{1,2\}}, 1_{m+1}}\left(l_{1, x}^{L}, l_{1, x}^{R}, \ldots, l_{m}\right) \\
& =\sum_{\pi \in \mathcal{P}_{m+1}: \pi \vee \overline{\{1,2\}}=1_{m+1}} N^{m-3} C_{\pi}\left(l_{1, x}^{L}, l_{1, x}^{R}, \ldots, l_{m}\right) \\
& =\sum_{\pi \in \mathcal{P}_{m+1}: \pi \vee\{1,2\}=1_{m+1}} N^{2 \# \pi-4} \Phi_{\pi}\left(l_{1, x}^{L}, l_{1, x}^{R}, \ldots, l_{m}\right)
\end{aligned}
$$

If $\pi \in \mathcal{P}_{m+1}$ satisfies $\pi \vee \overline{\{1,2\}}=1_{m+1}$, then, whether $\# \pi=1$ or $\# \pi=2$ and the Eq. (**) follows. Assume now that $x$ is an intersection point of $l_{1}$ with $l_{2}$. Then, for any partition $\pi \in \mathcal{P}_{m}, \mu_{x} \mathbb{E}_{\pi}\left(l_{1}, l_{2}, \ldots, l_{m}\right)=\frac{1}{N} \mathbb{E}_{\pi}\left(l_{1} \circ_{x} l_{2}, \ldots, l_{m}\right)$, if 1,2 are in the same block of $\pi$, and 0 otherwise, by Leibniz rule. This implies that for any partition $\pi \in \mathcal{P}_{m}$, such that 1 and 2 are not in the same block of $\pi, \mu_{x} C_{\pi}\left(l_{1}, l_{2}, \ldots, l_{m}\right)=0$. Therefore, for any partition $v \in \mathcal{P}_{m-1}$, denoting by $\tilde{v} \in \mathcal{P}_{m}$ the partition obtained by shifting $\pi$ by 1 and adding 1 to the block containing 2 ,

$$
\begin{aligned}
\sum_{\pi \leq \mathcal{v}} N \mu_{x} C_{\tilde{\pi}}\left(l_{1}, l_{2}, l_{3}, \ldots, l_{m}\right) & =\sum_{\mathcal{W} \leq \tilde{\nu}} N \mu_{x} C_{\mathcal{W}}\left(l_{1}, l_{2}, l_{3}, \ldots, l_{m}\right) \\
& =N \mu_{x} \mathbb{E}_{\tilde{\nu}}\left[l_{1}, l_{2}, \ldots, l_{m}\right]=\mathbb{E}_{\mathcal{v}}\left[l_{1} \circ_{x} l_{2}, l_{3}, \ldots, l_{m}\right] .
\end{aligned}
$$

It follows that for any $\pi \in \mathcal{P}_{m-1}, \mu_{x} C_{\tilde{\pi}}\left(l_{1}, l_{2}, \ldots, l_{m}\right)=\frac{1}{N} C_{\pi}\left(l_{1} \circ_{x} l_{2}, l_{3}, \ldots, l_{m}\right)$. For $\pi=1_{m-1}$, the latter equality yields

$$
\mu_{x} \Phi_{N}\left(l_{1}, l_{2}, \ldots, l_{m}\right)=\Phi_{N}\left(l_{1} \circ_{x} l_{2}, l_{3}, \ldots, l_{m}\right) .
$$

Observe that Eqs. $(*)$ and $(* * *)$ on $\Phi_{N}$ do not depend on $N$. 
7.2. Uniqueness for Makeenko-Migdal equations. We want to tackle this question by asking whether area-derivative operators can be obtained by linear combinations of the operators appearing on the left-hand-side of Theorem 12.

Therefor, we shall consider a slightly different notion of embedded graph. We call a multi-embedded graph in the plane a triplet $\mathbb{G}=(\mathbb{V}, \mathbb{E}, \mathbb{F})$ satisfying the same conditions as an embedded graph defined at the beginning of Sect. 6.2, without the condition of simple connectivity on the faces $\mathbb{F}$. The dual graph $\hat{\mathbb{G}}$ is then defined in the same way. Let us fix a regular skein $\mathcal{S}$ (Definition 1 of Sect. 6.7). We let $\mathbb{G}_{\mathcal{S}}=\left(\mathbb{V}_{\mathcal{S}}, \mathbb{E}_{\mathcal{S}}, \mathbb{F}_{\mathcal{S}}\right)$ be the finest multi-connected embedded graph such that $\mathcal{S} \subset \mathrm{P}\left(\mathbb{G}_{\mathcal{S}}\right)$. Let $\mathbb{E}^{+}$and $\lambda$ be respectively the orientation and the permutation of the edges $\mathbb{E}$ induced by $\mathcal{S}$. For any $N \geq 1$, the function $\Phi_{N}$ restricted to the class of $\mathcal{S}$ in $\overline{S k}_{r}\left(\mathbb{R}^{2}\right)$ can be considered as a smooth function on $\mathbb{R}_{+}^{\mathbb{F} \mathcal{S}}$ constant along the coordinate indexed by $F_{\infty}$. Let us set

$$
\begin{aligned}
\mu: \mathbb{R}^{\mathbb{F}} & \longrightarrow \mathbb{R}^{\mathbb{E}^{+}} \\
u & \longmapsto\left(e \mapsto u\left(F_{L}(e)\right)-u\left(F_{R}(e)\right)-u\left(F_{L}\left(\lambda^{-1}(e)\right)\right)+u\left(F_{R}\left(\lambda^{-1}(e)\right)\right)\right)
\end{aligned}
$$

and denote by $\mathfrak{m}$ its transpose. Identifying the vector space of first order differential operators on $C^{\infty}\left(\mathbb{R}_{+}^{\mathbb{F}}\right)$ with $\left(\mathbb{R}^{*}\right)^{\mathbb{F}}$, any operator $(*)$ and $(* *)$ defined in Theorem 12 is of the form $\mathfrak{m}\left(\partial_{e}\right), e \in \mathbb{E}^{+}$, where $\left(\partial_{e}\right)_{e \in \mathbb{E}^{+}}$denotes the canonical basis of $\left(\mathbb{R}^{*}\right)^{\mathbb{E}^{+}}$. To answer our question we need to identify the range of $\mathfrak{m}$. For any loop $l$ respectively in $\mathrm{L}(\mathbb{G})$ and $\mathcal{S}$, we denote by $n_{l} \in \mathbb{C}^{\mathbb{F}}$ and $\delta_{l} \in \mathbb{C}^{\mathbb{E}^{+}}$the winding number function of $l$ and the function $\sum_{e \in \mathbb{E}^{+}: l \text { traverses } e} \delta_{e}$. For any $v \in \mathbb{V}$, set

$$
*_{v}=\sum_{e \in \operatorname{Out}(v)} \delta_{e}
$$

where $\operatorname{Out}(v)$ denotes the set of oriented edges of $\mathbb{G}$, outgoing from $v$. The following lemma is proved in [35](Lemma 6.28.).

Lemma 20. (i) The kernel of $\mu$ is spanned by $\left\{n_{l_{1}}, n_{l_{2}}, \ldots, n_{l_{m}}, 1_{\mathbb{F}}\right\}$.

(ii) If $\mathbb{G}_{\mathcal{S}}$ is an embedded graph, then the image of $\mu$ is the orthogonal space to $\left\{*_{v}, v \in\right.$ $\mathbb{V}\} \cup\left\{\delta_{l}, l \in \mathcal{S}\right\}$.

Let us denote by $\left(\frac{d}{d|F|}\right)_{F \in \mathbb{F}}$ the canonical basis $\left(\mathbb{R}^{*}\right)^{\mathbb{F}}$. Note that we have a partial negative answer to our question: $\operatorname{dim}(\operatorname{ker}(\mu)) \geq 2$ and $\operatorname{Im}(\mathfrak{m})+\mathbb{R} \frac{d}{d\left|F_{\infty}\right|}=\operatorname{ker}(\mu)^{\perp}+\mathbb{R} \frac{d}{d\left|F_{\infty}\right|} \neq$ $\left(\mathbb{R}^{*}\right)^{\mathbb{F}}$. We need to complete the left-hand-side with a space of operators whose action on $\Phi(\mathcal{S})$ is known. When $m=1$, if $F_{0}$ is a face of $\mathbb{G}_{\mathcal{S}}$, neighbor of the infinite face, then, it is shown in [35] that $\mathbb{R} \frac{d}{d\left|F_{0}\right|}$ answers this question. In general, the following holds true.

Lemma 21. Suppose that $\mathbb{G}_{\mathcal{S}}$ is an embedded graph and that there exists $m$ distinct faces $F_{1}, \ldots, F_{m}$ of $\mathbb{G}_{\mathcal{S}}$, neighbors of the unbounded face such that for any $i \in[m], l_{i}$ is bounding $F_{i}$. Let $\mathbb{F}_{\infty, 1}=\left\{F_{\infty}, F_{1}, \ldots, F_{m}\right\}$. Then,

$$
\operatorname{Im}(\mathfrak{m}) \oplus \operatorname{span}\left\{\frac{d}{d|F|}: F \in \mathbb{F}_{\infty, 1}\right\}=\left(\mathbb{R}^{*}\right)^{\mathbb{F}}
$$


Proof. By assumption, for any $i, j \in[m], \frac{d}{d\left|F_{i}\right|}\left(n_{l_{j}}\right)=\delta_{i, j}$. As $\operatorname{Im}(\mathfrak{m})=\operatorname{ker}(\mu)^{\perp}$, Lemma 20 implies that $\operatorname{Im}(\mathfrak{m}) \cap \operatorname{span}\left(\frac{d}{d\left|F_{i}\right|}: i \in[m]\right)=\{0\}$ and $\operatorname{dim}(\operatorname{Im}(\mathfrak{m}))+m+1=$ $\# \mathbb{F}$.

Definition 3. We call a regular skein satisfying the condition of Lemma 21 a skein based at infinity.

For any skein based at infinity, the differential operators appearing in Theorem 12 allow to express any area derivative. Indeed according to the previous Lemma there exists ${ }^{10}$

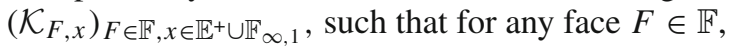

$$
\frac{d}{d|F|}=\sum_{e \in \mathbb{E}_{\mathcal{S}}^{+}} \mathcal{K}_{F, e} \mathfrak{m}\left(\partial_{e}\right)+\sum_{F^{\prime} \in \mathbb{F}_{\infty, 1}} \mathcal{K}_{F, F^{\prime}} \frac{d}{d\left|F^{\prime}\right|}
$$

Our task is now to show that solutions to the differential problem of Theorem 12 are characterized by their value on skeins based at infinity.

Remark 6. Note that if $\mathcal{S}$ satisfies the condition of Lemma 21 but the condition of simple connectivity, then one loop $l$ of $\mathcal{S}$ is disjoint from the others and under $\mathrm{YM}_{N}, H_{l}$ is independent from $\left(H_{l^{\prime}}\right)_{l^{\prime} \in \mathcal{S} \backslash\{l\}}$, so that $\Phi_{N}(\mathcal{S})=0$.

A problem is that the latter family of skeins is not stable by the operation (*): among the two skeins $\mathcal{S}_{v}^{L}$ and $\mathcal{S}_{v}^{R}$ obtained by splitting $\mathcal{S}$ at $v$, one of them might not be based at infinity. To solve this problem and compute the master field against all skeins, we could enlarge the type of loops families. Instead, we shall use that any skein can be deformed into a skein based at infinity, without changing the conjugacy class of its elements in $\mathrm{P}\left(\mathbb{R}^{2}\right)$.

For any $l \in \mathrm{P}\left(\mathbb{G}_{\mathcal{S}}\right)$, we consider

$$
d_{\infty, \mathcal{S}}(l)=\inf \left\{d_{\hat{\mathbb{G}}_{\mathcal{S}}}\left(F, F_{\infty}\right)-1: F \in \hat{\mathbb{G}}_{\mathcal{S}}, F \cap F_{\infty, \mathbb{G}_{\{l\}}}=\emptyset\right\} .
$$

Let $\mathbb{V}_{S}(\mathcal{S})$ and $\mathbb{V}_{f}(\mathcal{S})$ be respectively the points of self-intersection and of intersection of two different loops and set $I(\mathcal{S})=\# \mathbb{V}_{S}(\mathcal{S})+\# \mathbb{V}_{f}(\mathcal{S})$ (we shall drop the notation $\mathcal{S}$, when the context is not ambiguous). For any loop $l \in \mathcal{S}$, denote by $I_{\mathcal{S}}(l)$ the number of intersections of $l$ with itself and other loops of $\mathcal{S}$. We define the complexity of $\mathcal{S}$ to be the number

$$
\mathcal{C}(\mathcal{S})=I(\mathcal{S})+2 \sum_{l \in \mathcal{S}} d_{\infty, \mathcal{S}}(l)
$$

Example 2. A skein $\mathcal{S}$ is based at infinity if and only if $\mathcal{C}(\mathcal{S})=I(\mathcal{S})$.

Example 3. If $\mathcal{C}(\mathcal{S})=0$, then $\mathcal{S}$ is an union of closed Jordan curved bounding disjoints domains. Therefore, $\Phi(\mathcal{S})=0$, if \#S $\geq 2$ and $e^{-\frac{|D|}{2}}$, if $\mathcal{S}$ has a single loop bounding a simply connected domain $D$.

Recall that for any $v \in I_{\mathcal{S}}, \mathcal{S}_{v}$ denotes the transformed skein, whereas $l_{v}^{L}$ and $l_{v}^{R}$ stand for the two new loops of $\mathcal{S}_{v}$, when $v \in \mathbb{V}_{s}$, as respectively defined above Proposition 7 and Theorem 12.

\footnotetext{
10 We shall give an explicit formula in the next section.
} 
Lemma 22. (i) If $v \in \mathbb{V}_{f}(\mathcal{S})$,

$$
\mathcal{C}\left(\mathcal{S}_{v}\right)<\mathcal{C}(\mathcal{S})
$$

and if $v \in \mathbb{V}_{S}(\mathcal{S})$, for any partition $\mathcal{S}_{v}^{L} \sqcup \mathcal{S}_{v}^{R}=\mathcal{S}_{v}$, with $l_{v}^{L} \in \mathcal{S}_{v}^{L}$ and $l_{v}^{R} \in \mathcal{S}_{v}^{R}$,

$$
\max \left\{\mathcal{C}\left(\mathcal{S}_{v}^{L}\right), \mathcal{C}\left(\mathcal{S}_{v}^{R}\right)\right\}<\mathcal{C}(\mathcal{S}) .
$$

(ii) For any regular skein $\mathcal{S}$, there exists a family $\left(\mathcal{S}^{\epsilon}\right)_{\epsilon>0}$ of skeins based at infinity with $\mathcal{C}\left(\mathcal{S}^{\epsilon}\right)=\mathcal{C}(\mathcal{S})$, for any $\epsilon>0$, that converges to $\mathcal{S}^{\prime}$, with $\mathcal{S}^{\prime} \equiv \mathcal{S}$.

Proof. (i) Assume that $v \in \mathbb{V}_{f}$. Then, for any loop $l \in \mathcal{S}$, that does not contain $v$, $l \in \mathcal{S}_{v}$ and $d_{\infty, \mathcal{S}_{v}}(l) \leq d_{\infty, \mathcal{S}}(l)$. If $l_{1}$ and $l_{2}$ are the two loops crossing at $v$, then $d_{\infty, \mathcal{S}_{v}}\left(l_{1} \circ_{v} l_{2}\right) \leq \min \left\{d_{\infty, \mathcal{S}}\left(l_{1}\right), d_{\infty, \mathcal{S}}\left(l_{2}\right)\right\}$. Moreover, $\mathbb{V}_{s}\left(\mathcal{S}_{v}\right)=\mathbb{V}_{s}(\mathcal{S})$ and for any $w \in \mathbb{V}_{s}(\mathcal{S}), d_{\infty, \mathcal{S}_{v}}\left(l_{v}\right) \leq d_{\infty, \mathcal{S}}\left(l_{v}\right)$. Therefore, the fact that $I\left(\mathcal{S}_{v}\right)=I(\mathcal{S})-1$ yields the expected inequality.

Assume now that $v \in \mathbb{V}_{S}$. Let $l \in \mathcal{S}$ be the loop of $\mathcal{S}$ crossing at $v$ and fix a partition $\mathcal{S}_{v}^{L} \sqcup \mathcal{S}_{v}^{R}$ of $\mathcal{S}_{v}$ separating the loops $l_{v}^{L}$ and $l_{v}^{R}$. For any loop $l^{\prime} \in \mathcal{S}_{v} \backslash\left\{l_{v}^{L}, l_{v}^{R}\right\}$, $d_{\infty, \mathcal{S}_{v}^{L}}\left(l^{\prime}\right), d_{\infty, \mathcal{S}_{v}^{R}}\left(l^{\prime}\right) \leq d_{\infty, \mathcal{S}}\left(l^{\prime}\right)$, whereas $\min _{a \in\{L, R\}} d_{\infty, \mathcal{S}}\left(l_{v}^{a}\right)=d_{\infty, \mathcal{S}}(l)$. Let us suppose w.l.o.g. that the latter minimum is reached at $a=L$, and let $c \in \mathrm{P}\left(\hat{\mathbb{G}}_{\mathcal{S}}\right)$ be a path such that $F_{\infty, \mathbb{G}_{\left\{l_{v}^{L}\right\}}} \cap \underline{c}=\emptyset$ and $|c|-1=d_{\infty, \mathcal{S}}\left(l_{v}^{L}\right)=d_{\infty, \mathcal{S}}(l)$. Then, $I\left(\mathcal{S}_{v}^{L}\right) \leq I\left(\mathcal{S}_{v}\right)-1$ and as $d_{\infty, \mathcal{S}_{v}^{L}}\left(l_{v}^{L}\right) \leq d_{\infty, \mathcal{S}}(l), \mathcal{C}\left(\mathcal{S}_{v}^{L}\right) \leq \mathcal{C}(\mathcal{S})-1$. The right side needs more caution. Let us consider the two paths $c^{ \pm} \in \mathrm{P}\left(\hat{\mathbb{G}}_{\mathcal{S}_{v}^{R}}\right)$ induced by $l_{v}^{L}$ and $c$ in the following way: $\underline{c}^{ \pm}$is the face on the left of the outgoing edge of $l_{v}^{R}$ at $v$, one path follows the orientation of $l_{v}^{L}$ and the other goes in the reverse direction, erasing loops chronologically, until they first hit $c$, when they both follow $c$ up to $F_{\infty, \mathbb{G}_{\mathcal{S}_{v}}}$. Their combinatorial length satisfies

$$
\left|c^{+}\right|+\left|c^{-}\right| \leq I_{\mathcal{S}_{v}}\left(l_{v}^{L}\right)+2(|c|-1) .
$$

Therefore,

$$
d_{\infty, \mathcal{S}^{R}}\left(l_{v}^{R}\right) \leq \min \left\{\left|c^{+}\right|,\left|c^{-}\right|\right\} \leq \frac{I_{\mathcal{S}_{v}}\left(l_{v}^{L}\right)}{2}+d_{\infty, \mathcal{S}}(l)
$$

The number of intersections of $\mathcal{S}_{v}^{R}$ is bounded by $I(\mathcal{S} \backslash\{l\})+I_{\mathcal{S}_{v}}\left(l_{v}^{R}\right)$. Moreover, for any loop $l^{\prime} \in \mathcal{S}_{v}^{R} \backslash\left\{l_{v}^{R}\right\}, d_{\infty, \mathcal{S}_{v}^{R}}\left(l^{\prime}\right) \leq d_{\infty, \mathcal{S}}\left(l^{\prime}\right)$. It follows that

$$
\mathcal{C}\left(\mathcal{S}_{v}^{R}\right) \leq I(\mathcal{S} \backslash\{l\})+I_{\mathcal{S}_{v}}\left(l_{v}^{R}\right)+I_{\mathcal{S}_{v}}\left(l_{v}^{L}\right)+2 \sum_{l^{\prime} \in \mathcal{S}} d_{\infty, \mathcal{S}}\left(l^{\prime}\right) .
$$

The equality $I_{\mathcal{S}_{v}}\left(l_{v}^{R}\right)+I_{\mathcal{S}_{v}}\left(l_{v}^{L}\right)+I(\mathcal{S} \backslash\{l\})=I\left(\mathcal{S}_{v}\right)=I(\mathcal{S})-1$ implies the claim.

(ii) For each $l \in \mathcal{S}$, such that $d_{\infty, \mathcal{S}}(l)>0$, consider a self-avoiding path $c_{l}$ in $\hat{\mathbb{G}}_{\mathcal{S}}$, such that $F_{\infty, \mathbb{G}_{l}} \cap \underline{c}_{l}=\emptyset, \bar{c}_{l}=F_{\infty, \mathbb{G}_{\mathcal{S}}}$ and $\left|c_{l}\right|=d_{\infty, \mathcal{S}}(l)+1$. Choose such a family $\left(c_{l}\right)_{l \in \mathcal{S}}$ of loops that do not cross each other but may be merged with one another. Deform each loop $l$ along $c_{l}$ into $\tilde{l}$ so that the deformation intersects exactly twice each dual edge of $c_{l}$ and does not intersect the deformation of other loops. Denote by $\tilde{\mathcal{S}}$ the 
skein $\left\{\tilde{l}: l \in \mathcal{S}, d_{\infty, \mathcal{S}}(l)>0\right\} \cup\left\{l: l \in \mathcal{S}, d_{\infty, \mathcal{S}}(l)=0\right\}$. By construction, for any $l \in \tilde{\mathcal{S}}, d_{\infty, \tilde{\mathcal{S}}}(l)=0$ and

$$
\mathcal{I}(\tilde{\mathcal{S}})=I(\mathcal{S})+2 \sum_{l \in \mathcal{S}}\left(\left|c_{l}\right|-1\right)=\mathcal{C}(\mathcal{S}) .
$$

We can now choose a family of skeins $\left(\mathcal{S}^{\epsilon}\right)_{\epsilon>0}$ as above, that converges towards a skein $\mathcal{S}^{\prime}$, such that $\mathcal{S}^{\prime} \equiv \mathcal{S}$, as $\epsilon \rightarrow 0$.

We can now solve our differential system recursively ordering skeins by their complexity. Recall that if $x$ is a point of intersection of a skein $\mathcal{S}$, then $\mu_{x}=\frac{d}{d\left|F_{1}\right|}-\frac{d}{d\left|F_{2}\right|}+$ $\frac{d}{d\left|F_{3}\right|}-\frac{d}{d\left|F_{4}\right|}$, where $F_{1}, F_{2}, F_{3}$ and $F_{4}$ are faces around the vertex $v$ in cyclic order and $F_{1}$ is the face bounded by the two outgoing edges of $x$.

Theorem 13. There exists a unique function $\Phi$ on $\operatorname{Sk}\left(\mathbb{R}^{2}\right)$ satisfying the following equations: ${ }^{11}$

1. $\Phi(\{1\})=1$.

2. If $\mathcal{S}^{-}$and $\mathcal{S}^{+}$are two skeins that are separated by a closed Jordan curve, $\Phi\left(\mathcal{S}^{-} \sqcup\right.$ $\left.\mathcal{S}^{+}\right)=0$.

3. $\Phi$ is continuous.

4. If $\mathcal{S} \equiv \mathcal{S}^{\prime}$ (definition on p. 40), then $\Phi\left(\mathcal{S}^{\prime}\right)=\Phi(\mathcal{S})$.

5. For any area-preserving diffeomorphism $g$ of the plane, $\Phi \circ g=\Phi$.

6. For any regular skein $\mathcal{S}, \Phi$ is differentiable with respect to $(|F|)_{F \in \mathbb{F}_{S}}$ and satisfies the following differential equations. If $x$ is the intersection of two different loops,

$$
\mu_{x} \Phi(\mathcal{S})=\Phi\left(\mathcal{S}_{x}\right)
$$

If $x$ is the intersection of a loop $l$ of $\mathcal{S}$ with itself,

$$
\mu_{x} \Phi(\mathcal{S})=\sum_{\substack{\mathcal{S}_{x}^{L} \sqcup \mathcal{S}_{x}^{R}=\mathcal{S}_{x} \\ l_{x}^{L} \in \mathcal{S}_{x}^{L} \text { and } l_{x}^{R} \in \mathcal{S}_{x}^{R}}} \Phi_{N}\left(\mathcal{S}_{x}^{L}\right) \Phi_{N}\left(\mathcal{S}_{x}^{R}\right) .
$$

For any face $F \in \mathbb{F}_{\mathcal{S}}$, neighbor of $F_{\infty}$,

$$
\frac{d}{d|F|} \Phi(\mathcal{S})=-\frac{1}{2} \Phi(\mathcal{S}) .
$$

Proof. The function $\Phi_{N}$ satisfies by construction the point 1-5. According to Theorems 12 and 9 , for any regular skein $\mathcal{S}, \Phi_{N}(\mathcal{S})$ is analytic in $(|F|)_{F \in \mathbb{F}_{\mathcal{S}}}$, satisfies $(*),(* *)$ and $(* * *)$ and converges uniformly on every compact set of $\mathbb{R}_{+}^{\mathbb{F}} \mathcal{S}$ to the function $\Phi(\mathcal{S})$. Therefore, $\Phi(\mathcal{S})$ is analytic and satisfies the equations of point 6 . It remains to show uniqueness of the solutions of the latter problem. We wish to prove it by induction on the complexity (45). Let $\Psi$ be a function on finite skeins satisfying point 1 to 6 . Using point 3, it is enough to prove that $\Psi(\mathcal{S})=\Phi(\mathcal{S})$ for any regular skein $\mathcal{S}$. For any integer $n$, set $\mathrm{Sk}_{n}=\left\{\mathcal{S} \in \mathrm{Sk}_{r}\left(\mathbb{R}^{2}\right): \mathcal{C}(\mathcal{S}) \leq n\right\}$. Let us prove inductively that $\Psi_{\mid S k_{n}}=\Phi_{\mid S k_{n}}$. Thanks to points 1 and 2 , the equality holds for $n=0$. Assume that it is true for $n \in \mathbb{N}$ and consider a regular skein $\mathcal{S} \in \mathrm{Sk}_{n+1}$. Suppose that $\mathcal{S}$ is based at infinity. According to the inversion formula (44) together with point 6 and Lemma 22,

11 The set of skein is defined as in Sect. 6.7 with the topology associated to $d_{\ell}$ (defined in Sect. 6.4). 
for any face $F \in \mathbb{F}_{\mathcal{S}}, \frac{d}{d|F|} \Phi$ and $\frac{d}{d|F|} \Psi$ are a linear combination of terms of the form $\Phi\left(\mathcal{S}^{\prime}\right), \Psi\left(\mathcal{S}^{\prime}\right)$ or $\Phi\left(\mathcal{S}^{L}\right) \Phi\left(\mathcal{S}^{R}\right), \Psi\left(\mathcal{S}^{L}\right) \Psi\left(\mathcal{S}^{R}\right)$, with $\mathcal{C}\left(\mathcal{S}^{\prime}\right), \mathcal{C}\left(\mathcal{S}^{L}\right), \mathcal{C}\left(\mathcal{S}^{R}\right)<n$. Hence, by induction hypothesis, $\Psi(\mathcal{S})=\Phi(\mathcal{S})$. Assume now that $\mathcal{S}$ is not based at infinity. Let $\left(\mathcal{S}^{\epsilon}\right)_{\epsilon>0}$ be given as in Lemma 22. Then, for any $\epsilon>0, \mathcal{S}^{\epsilon} \in \mathrm{Sk}_{n+1}$ is based at infinity and $\Psi\left(\mathcal{S}^{\epsilon}\right)=\Phi\left(\mathcal{S}^{\epsilon}\right)$. The points 3 and 4 yield that $\Psi(\mathcal{S})=\Phi(\mathcal{S})$.

7.3. Generalized Kazakov basis. We consider a skein $\mathcal{S}$ based at infinity ${ }^{12}$ and set $\mathbb{G}=\mathbb{G}_{\mathcal{S}}$. We shall give here bases adapted to the decomposition $\operatorname{Im}(\mathfrak{m}) \oplus \operatorname{span}\left\{\frac{d}{d|F|}\right.$ : $\left.F \in \mathbb{F}_{\infty, 1}\right\}$ and their dual, which leads to an explicit formula for the matrix $\mathcal{K}$ appearing in (44). We call them Kazakov bases, following the works [31,35], where they were introduced in the case $\# \mathcal{S}=1$.

For any $l \in \mathcal{S}$, let $\tilde{l}$ and $e_{l} \in \mathbb{E}$ be the non-based loop associated to $l$ and the edge dual to $\left(F_{\infty}, F_{l}\right)$, where $\mathbb{F}_{\infty, 1}=\left\{F_{l}, l \in \mathcal{S}\right\} \cup\left\{F_{\infty}\right\}$. For any pair $l, l^{\prime} \in \mathcal{S}$ of distinct intersecting loops, let us fix a vertex $p_{l, l^{\prime}} \in \mathbb{V}_{f}$ at the intersection of $l, l^{\prime}$, and set $\mathbb{V}_{0}$ the collection of these points. We denote by $\prec$ an arbitrary order on $\mathcal{S}$. A Kazakov basis is described thanks to families of loops in $\mathrm{L}(\mathbb{G})$. For any $v \in \mathbb{V} \backslash \mathbb{V}_{0}$, we define a loop $l_{v}$ based at $v$ as follows:

(i) If $v \in \mathbb{V}_{s}, l_{v}$ is the restriction of $l$ between two visits of $v$ that does not use $e_{l}$.

(ii) If $v \in \mathbb{V}_{f} \backslash \mathbb{V}_{0}$ is at the intersection of $l, l^{\prime} \in \mathcal{S}$ with $l \prec l^{\prime}, l_{v}$ is the concatenation of the restrictions of $\tilde{l}$ or $\tilde{l}^{-1}$ between the hitting time of $v$ and $p_{l, l^{\prime}}$, with the restriction of $\tilde{l}^{\prime}$ between the hitting time of $p_{l, l^{\prime}}$ and $v$, that does not use the edges $e_{l}$ and $e_{l^{\prime}}$.

Consider the graph $\mathcal{G}_{\mathcal{S}}$ with vertices indexed by $\mathcal{S}$, such that two loops are connected in $\mathcal{G}_{\mathcal{S}}$ if and only if they intersect each other. For any $v \in \mathbb{V}$, let $e(v)$ be the left-outgoing edge at $v$. The winding number of a non-backtracking loop $l \in \mathrm{L}(\mathbb{G})$ jumps by 1 along any dual edge of $\mathbb{G}$ that crosses $l$ from right to left. Hence, for any loop $l \in \mathrm{L}_{v}(\mathbb{G})$, using exactly two edges around $v$, bounding the same face,

$$
\epsilon(l)=\mathfrak{m}\left(\partial_{e(v)}\right)\left(n_{l}\right) \in\{-1,1\},
$$

whereas for any $l \in \mathcal{S}$,

$$
\epsilon_{l}=\frac{d}{d\left|F_{l}\right|}\left(n_{l}\right) \in\{-1,1\}
$$

Lemma 23. If $\mathcal{G}_{\mathcal{S}}$ is a tree,

$$
\beta=\left\{\mathfrak{m}\left(\partial_{e(v)}\right), e \in \mathbb{V} \backslash \mathbb{V}_{0}\right\} \cup\left\{\frac{d}{d|F|}-\frac{d}{d\left|F_{\infty}\right|}: F \in \mathbb{F}_{\infty, 1}\right\} \cup\left\{\frac{d}{d\left|F_{\infty}\right|}\right\}
$$

is a basis of $\left(\mathbb{R}^{*}\right)^{\mathbb{F}}$, with dual

$$
\alpha=\left\{\epsilon\left(l_{v}\right) n_{l_{v}}: v \in \mathbb{V} \backslash \mathbb{V}_{0}\right\} \cup\left\{\epsilon_{l} n_{l}: l \in \mathcal{S}\right\} \cup\left\{1_{\mathbb{F}}\right\} .
$$

Proof. For any loop $l$ in $\mathrm{L}(\mathbb{G})$ and $e \in \mathbb{E}^{+}$, such that $l$ uses exactly $e$ and $\lambda^{-1}(e)$ among the four edges adjacent to $\underline{e}, \mathfrak{m}\left(\partial_{e}\right)\left(n_{l}\right)=0$. Besides for any loop $l$ belonging to the families (i) or (ii), and any $a, b \in \mathcal{S}, \frac{d}{d\left|F_{a}\right|}\left(n_{l}\right)=0$, as $l$ does not use $e_{a}$, for any $v \in \mathbb{V} \backslash \mathbb{V}_{0}, \mathfrak{m}\left(\delta_{e(v)}\right)\left(n_{l}\right)=\epsilon_{v}(l) \delta_{v, \underline{l}}, \frac{d}{d\left|F_{a}\right|} n_{b}=\epsilon_{a} \delta_{a, b}, \frac{d}{d\left|F_{\infty}\right|}\left(n_{l}\right)=0$ and

12 Recall Definition 3 of the last section. 


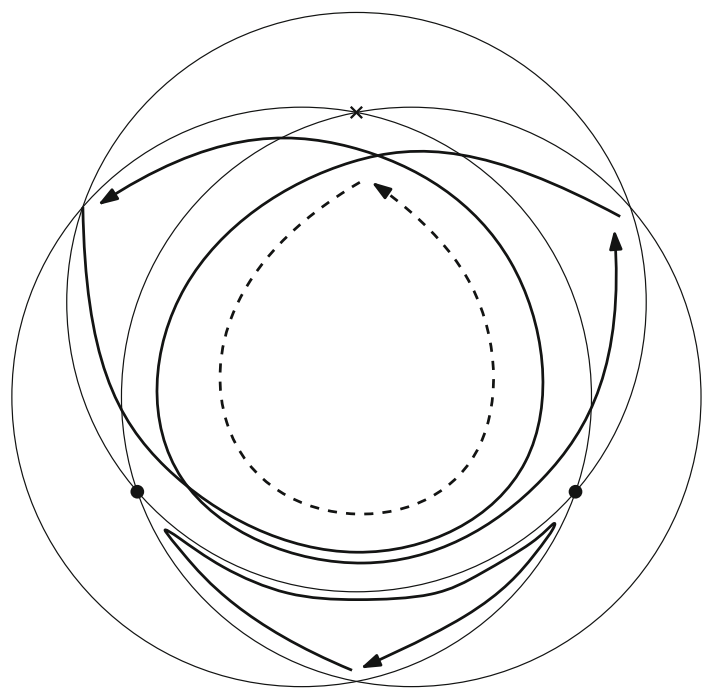

Fig. 7. A Kazakov basis for three circles with counterclockwise orientation. The set $\mathbb{V}_{0}$ is given by vertices of the inner triangle and $\mathbb{V}(\mathfrak{T})$ is drawn with black disks. There are no type (i) loops and type (ii) and (iii) loops are drawn respectively with plain and dashed lines

$\frac{d}{d|F|}\left(1_{\mathbb{F}}\right)=1$, for any face $F \in \mathbb{F}$. It follows that the two families $\alpha$ and $\beta$ are free and dual to each other, with rank $\# \mathbb{V}-\# \mathbb{V}_{0}+\# \mathcal{S}+1$. Any vertex of $\mathbb{G}$ has degree 4 , hence by Euler's relation, $\# \mathbb{V}=\# \mathbb{F}-2$. Besides, there are as many points in $\mathbb{V}_{0}$ as there are couples of distinct intersecting loops, therefore $\mathbb{V}_{0}$ is the number of edges of $\mathcal{G}_{\mathcal{S}}$. If $\mathcal{G}_{\mathcal{S}}$ is a tree, $\# \mathbb{V}_{0}=\# \mathcal{S}-1$ and the rank of $\alpha$ is $\# \mathbb{F}$.

If $\mathcal{G}_{\mathcal{S}}$ is not a tree the latter family is not a basis anymore and can be modified as follows. Let $\mathfrak{T}$ and $E_{\mathcal{S}}^{+}$be a spanning tree of $\mathcal{G}_{\mathcal{S}}$ and an arbitrary orientation of $\mathcal{G}_{\mathcal{S}}$ and denote by $\mathbb{V}(\mathfrak{T})$, the set of vertices at the intersection of a pair of loops $\left(l, l^{\prime}\right)$ that is an edge of $\mathfrak{T}$.

(i) and (ii) For $v \in \mathbb{V}_{s} \cup\left(\mathbb{V}_{f} \cap \mathbb{V}(\mathfrak{T})\right)$, the definition of $l_{v}$ is not changed.

(iii) Let $v \in \mathbb{V}_{f} \backslash \mathbb{V}(\mathfrak{T})$ be an intersection point of two loops $l, l^{\prime}$, such that $\left(l, l^{\prime}\right) \in E_{\mathcal{S}}^{+}$ is not an edge of $\mathfrak{T}$. Let us consider the path $l_{0}, l_{1}, \ldots, l_{m}$ in $\mathfrak{T}$, with $l_{0}=l$ and $l_{m}=l^{\prime}$, and set $p_{0}=v=p_{m+1}$ and $p_{k}=p_{l_{k-1}, l_{k}}$, for $1 \leq k \leq m$. For any $0 \leq k \leq m$, fix a path $\gamma_{k}$ from $p_{k}$ to $p_{k+1}$, restriction of $\tilde{l}_{k}$ or $\tilde{l}_{k}^{-1}$, that is not using $e_{l_{k}}$. We define a loop based at $v$ setting $l_{v}=\gamma_{0} \gamma_{1} \ldots \gamma_{m}$. See Fig. 7 for an example.

Lemma 24. The family

$$
\beta^{\prime}=\left\{\mathfrak{m}\left(\partial_{e(v)}\right), v \in \mathbb{V} \backslash\left(\mathbb{V}_{0} \cap \mathbb{V}(\mathfrak{T})\right)\right\} \cup\left\{\frac{d}{d|F|}-\frac{d}{d\left|F_{\infty}\right|}: F \in \mathbb{F}_{\infty, 1}\right\} \cup\left\{\frac{d}{d\left|F_{\infty}\right|}\right\}
$$

is a basis of $\left(\mathbb{R}^{*}\right)^{\mathbb{F}}$ with dual

$$
\alpha^{\prime}=\left\{\epsilon\left(l_{v}\right) n_{l_{v}}: v \in \mathbb{V} \backslash\left(\mathbb{V}_{0} \cap \mathbb{V}(\mathfrak{T})\right)\right\} \cup\left\{\epsilon_{l} n_{l}: l \in \mathcal{S}\right\} \cup\left\{1_{\mathbb{F}}\right\} .
$$

Proof. If $a$ and $b$ are two distinct loops of the families (i), (ii) or (iii), whether $b$ uses the outgoing edge $e \in \operatorname{Out}(\underline{a})$ and $\lambda^{-1}(e)$ and no other edge around $v$, or it does go 
through $v$, in both cases, $\mathfrak{m}\left(\partial_{e(\underline{a})}\right)\left(n_{b}\right)=0$. As $b$ does not use any edge in $\left\{e_{l}, l \in \mathcal{S}\right\}$, for any $F \in \mathbb{F}_{\infty, 1}, \frac{d}{d|F|}\left(n_{b}\right)=0$. It follows that $\alpha^{\prime}$ and $\beta^{\prime}$ are free and dual to each other. Besides, $\# \mathbb{V}_{0} \cap \mathbb{V}(\mathfrak{T})$ is the number of edges of $\mathfrak{T}$, that is, $\# \mathcal{S}-1$. Therefore, $\alpha^{\prime}, \beta^{\prime}$ have $\operatorname{rank} \# \mathbb{V}-\#\left(\mathbb{V}_{0} \cap \mathbb{V}(\mathfrak{T})\right)+\# \mathcal{S}+1=\# \mathbb{V}+2=\# \mathbb{F}$.

We have now an explicit expression for $\mathcal{K}$ in (44). For any regular skein $\mathcal{S}$ based at infinity and any bounded face $F \in \mathbb{F}_{\mathcal{S}}$,

$$
\frac{d}{d|F|}=\sum_{v \in \mathbb{V} \backslash\left(\mathbb{V}_{0} \cap \mathbb{V}(\mathfrak{T})\right)} \epsilon\left(l_{v}\right) n_{l_{v}}(F) \mathfrak{m}\left(\partial_{e(v)}\right)+\sum_{l \in \mathcal{S}} \epsilon_{l} n_{l}(F) \frac{d}{d\left|F_{l}\right|}
$$

Acknowledgements. The author wishes to thank his Ph.D. advisor Thierry Lévy, as well as Franck Gabriel and Guillaume Cébron, for fruitful discussions about Yang-Mills measure and the planar master field. Many thanks are due to the anonymous referee for useful comments. This research work has been partly funded by the RTG 1845 and the EPSRC Grant New Frontiers in Random Geometry.

Open Access This article is distributed under the terms of the Creative Commons Attribution 4.0 International License (http://creativecommons.org/licenses/by/4.0/), which permits unrestricted use, distribution, and reproduction in any medium, provided you give appropriate credit to the original author(s) and the source, provide a link to the Creative Commons license, and indicate if changes were made.

\section{References}

1. Anshelevich, M., Sengupta, A.N.: Quantum free Yang-Mills on the plane. J. Geom. Phys. 62(2), 330343 (2012)

2. Banchoff, T.F., Pohl, W.F.: A generalization of the isoperimetric inequality. J. Differ. Geom. 6, 175-192 (1971/72)

3. Benaych-Georges, F.: Central limit theorems for the Brownian motion on large unitary groups. Bull. Soc. Math. Fr. 139(4), 593-610 (2011)

4. Bernadi, O., Morales, A.H.: Counting trees using symmetries. J. Combin. Theory Ser. A 123(1), 104$122(2014)$

5. Biane, P.: Free Brownian Motion, Free Stochastic Calculus and Random Matrices. In: Free Probability Theory (Waterloo, ON, 1995), Vol. 12 of Fields Inst. Commun., pp. 1-19. Amer. Math. Soc., Providence, RI (1997)

6. Biane, P.: Segal-Bargmann transform, functional calculus on matrix spaces and the theory of semi-circular and circular systems. J. Funct. Anal. 144(1), 232-286 (1997)

7. Borot, G., Guionnet, A.: Asymptotic expansion of $\beta$ matrix models in the one-cut regime. Commun. Math. Phys. 317(2), 447-483 (2013)

8. Brézin, E., Itzykson, C., Parisi, G., Zuber, J.B.: Planar diagrams. Commun. Math. Phys. 59(1), 3551 (1978)

9. Cébron, G., Kemp, T.: Fluctuations of Brownian Motions on $G L_{N}$ (2014). arXiv:1409.5624

10. Cébron, G., Dahlqvist, A., Gabriel, F.: The Generalized Master Fields (2016). arXiv:1601.00214

11. Collins, B.: Moments and cumulants of polynomial random variables on unitary groups, the ItzyksonZuber integral, and free probability. Int. Math. Res. Not. 17, 953-982 (2003)

12. Collins, B., Dahlqvist, A., Kemp, T.: Strong Convergence of Unitary Brownian Motion (2015). arXiv: 1502.06186

13. Collins, B., Guionnet, A., Maurel-Segala, E.: Asymptotics of unitary and orthogonal matrix integrals. Adv. Math. 222(1), 172-215 (2009)

14. Collins, B., Mingo, J.A., Śniady, P., Speicher, R.: Second order freeness and fluctuations of random matrices. III. Higher order freeness and free cumulants. Doc. Math. 12, 1-70 (2007)

15. Collins, B., Śniady, P.: Representations of Lie groups and random matrices. Trans. Am. Math. Soc. 361(6), 3269-3287 (2009)

16. Dahlqvist, A.: Integration formula for Brownian motion on classical compact lie groups. To appear in Annales de l'Institut Henri Poincaré (B) Probabilités et Statistiques (2016)

17. Diaconis, P., Evans, S.N.: Linear functionals of eigenvalues of random matrices. Trans. Am. Math. Soc. 353(7), 2615-2633 (2001)

18. Diaconis, P., Shahshahani, M.: On the eigenvalues of random matrices. J. Appl. Prob. 31(3), 49-62 (1994) 
19. Driver, B.K.: $\mathrm{YM}_{2}$ : continuum expectations, lattice convergence, and lassos. Commun. Math. Phys. 123(4), 575-616 (1989)

20. Driver, B.K., Hall, B.C., Kemp, T.: Three Proofs of the Makeenko-Migdal Equation for Yang-Mills Theory on the Plane (2016). arXiv:1601.06283

21. Driver, B.K., Gabriel, F., Hall, B.C., Kemp, T.: The Makeenko-Migdal Equation for Yang-Mills Theory on Compact Surfaces (2016). arXiv:1602.03905

22. Féray, V., Méliot, P.-L., Nikeghbali, A.: Mod-phi Convergence and Precise Deviations (2013). arXiv:1304.2934v4

23. Gabriel, F.: Planar Markovian Holonomy Fields (2015). arXiv:1501.05077

24. Goulden, I.P., Guay-Paquet, M., Novak, J.: Monotone Hurwitz numbers and the HCIZ integral. Ann. Math. Blaise Pascal 21(1), 71-89 (2014)

25. Goulden, I.P., Guay-Paquet, M., Novak, J.: Monotone Hurwitz numbers in genus zero. Can. J. Math. 65(5), 1020-1042 (2013)

26. Gross, D.J., Matytsin, A.: Some properties of large- $N$ two-dimensional Yang-Mills theory. Nucl. Phys. B 437(3), 541-584 (1995)

27. Guionnet, A., Novak, J.: Asymptotics of unitary multimatrix models: the Schwinger-Dyson lattice and topological recursion (English summary). J. Funct. Anal. 268(10), 2851-2905 (2015)

28. Harish-Chandra: Differential operators on a semisimple Lie algebra. Am. J. Math. 79, 87-120 (1957)

29. G. 't Hooft: A planar diagram theory for strong interactions. Nucl. Phys. B 72, 461-473 (1974)

30. Humphries, S.P.: On weakly distinguished bases and free generating sets of free groups. Q. J. Math. 36(2), 215-219 (1985)

31. Kazakov, V.A.: Wilson loop average for an arbitrary contour in two-dimensional u(n) gauge theory. Nucl. Phys. B 179(2), 283-292 (1981)

32. Kazakov, V.A., Kostov, I.K.: Computation of the wilson loop functional in two-dimensional u( $\infty)$ lattice gauge theory. Phys. Lett. B 105(6), 453-456 (1981)

33. Leonov, V.P., Shiryaev, A.N.: On a method of semi-invariants. Theor. Probab. Appl. 4, 319-329 (1959)

34. Lévy, T.: Schur-Weyl duality and the heat kernel measure on the unitary group. Adv. Math. 218(2), 537575 (2008)

35. Lévy, T.: The Master Field on the Plane (2011). arXiv:1112.2452

36. Lévy, T., Maïda, M.: Central limit theorem for the heat kernel measure on the unitary group. J. Funct. Anal. 259(12), 3163-3204 (2010)

37. Lévy. T.: Two-Dimensional Markovian Holonomy Fields. Société Mathématique de France, Vol. 329. Astérisque (2010)

38. Makeenko, Y.M., Migdal, A.A.: Exact equation for the loop average in multicolor qcd. Phys. Lett. B 88, 135-137 (1979)

39. Méliot, P.-L.: The cut-off phenomenon for Brownian motions on symmetric spaces of compact type. Potential Anal. 40(4), 427-509 (2014)

40. Rains, E.M.: Combinatorial properties of Brownian motion on the compact classical groups. J. Theor. Probab. 10(3), 659-679 (1997)

41. Rota, G.C.: On the foundations of combinatorial theory. I. Theory of Möbius functions. Z. Wahrscheinlichkeitstheorie Und Verw. Gebiete 2, 340-368 (1964)

42. Sengupta, A.N.: Gauge theory on compact surfaces. Mem. Am. Math. Soc. 126(600), viii+85 (1997)

43. Singer, I.M.: In: Simon, G., James, L., Robert, L. W. (eds.) Functional Analysis on the Eve of the 21st Century. Vol. I, vol. 131 of Progress in Mathematics, p. 263. Birkhäuser Boston Inc., Boston, MA, 1995. In honor of the eightieth birthday of I. M. Gel'fand, Papers from the conference held at Rutgers University, New Brunswick, New Jersey, October 24-27 (1993)

44. Ikeda, N., Watanabe S.: Stochastic Differential Equations and Diffusion Processes, Vol. 24, North-Holland Mathematical Library. North-Holland Publishing Co., Amsterdam; Kodansha, Ltd., Tokyo (1981)

45. Xu, F.: A random matrix model from two-dimensional Yang-Mills theory. Commun. Math. Phys. 190(2), 287-307 (1997)

46. Zinn-Justin, P., Zuber, J.-B.: On some integrals over the $\mathrm{U}(N)$ unitary group and their large $N$ limit. J. Phys. A 36(12), 3173-3193 (2003)

47. Zvonkin, A.: Matrix integrals and map enumeration: an accessible introduction. Math. Comput. Model. 26(8-10), 281-304 (1997)

Communicated by S. Zelditch 